\title{
Complex photonics: Dynamics and applications of delay-coupled semiconductors lasers
}

\author{
Miguel C. Soriano \\ Instituto de Física Interdisciplinar y Sistemas Complejos IFISC, Campus Universitat de les \\ Illes Balears, 07122 Palma de Mallorca, Spain \\ Jordi García-Ojalvo* \\ Department of Experimental and Health Sciences, Universitat Pompeu Fabra, Barcelona \\ Biomedical Research Park, Dr. Aiguader 88, 08003 Barcelona, Spain \\ Claudio R. Mirasso and Ingo Fischer ${ }^{\dagger}$ \\ Instituto de Física Interdisciplinar y Sistemas Complejos IFISC (UIB-CSIC), Campus \\ Universitat de les Illes Balears, 07122 Palma de Mallorca, Spain
}

(published 20 March 2013)

\begin{abstract}
Complex phenomena in photonics, in particular, dynamical properties of semiconductor lasers due to delayed coupling, are reviewed. Although considered a nuisance for a long time, these phenomena now open interesting perspectives. Semiconductor laser systems represent excellent test beds for the study of nonlinear delay-coupled systems, which are of fundamental relevance in various areas. At the same time delay-coupled lasers provide opportunities for photonic applications. In this review an introduction into the properties of single and two delay-coupled lasers is followed by an extension to network motifs and small networks. A particular emphasis is put on emerging complex behavior, deterministic chaos, synchronization phenomena, and application of these properties that range from encrypted communication and fast random bit sequence generators to bioinspired information processing.
\end{abstract}

DOI: $10.1103 /$ RevModPhys.85.421

PACS numbers: 42.65.Sf, 05.45.Xt, 42.55.Px, 05.45.Gg

\section{CONTENTS}

I. Introduction: Past and Vision

422

A. Context: Semiconductor lasers $\quad 422$

B. Instabilities as a nuisance

C. Semiconductor lasers as test beds for nonlinear dynamics studies

D. Potential of complex photonics

II. Dynamics of Semiconductor Lasers with

Delayed Self-Coupling

A. Some history of lasers with delayed optical feedback

B. Consequences of delayed self-coupling

C. Semiconductor laser nonlinearities

D. Phenomena and mechanisms of delayed optical feedback

1. Long delay regime

2. Short delay regime

E. Multimode effects

430

1. Longitudinal modes

430

2. Transverse modes: Spatiotemporal instabilities 431

F. Other feedback schemes

431

1. Frequency-selective feedback

2. Polarization-rotated feedback

431

431

\footnotetext{
* On leave from Departament de Física i Enginyeria Nuclear, Universitat Politècnica de Catalunya, Edifici GAIA, Rambla de Sant Nebridi s/n, 08222 Terrassa, Barcelona, Spain.

†ingo@ifisc.uib-csic.es
}

3. Optoelectronic feedback 432

4. Phase-conjugate feedback 432

G. Influence of noise $\quad 433$

1. Noise robustness 433

2. Ordering role of noise 433

H. Advances and challenges in acquisition and characterization of semiconductor laser dynamics $\quad 434$

III. Dynamics and Synchronization of Delay-Coupled Semiconductor Lasers

A. Identical synchronization, generalized synchronization, and consistency

B. Unidirectional coupling

C. Dynamics and synchronization of two bidirectionally coupled semiconductor lasers

D. Dynamics and synchronization of further bidirectionally coupled semiconductor laser configurations

1. Mutually coupled lasers subject to self-feedback 440

2. Mutually coupled lasers subject to a common drive

3. Mutually coupled lasers through

a relay element

E. Stability analysis of the identical (zero-lag) solution 442

F. Ordering role of noise

IV. Network Motifs and Small Networks of

Delay-Coupled Semiconductor Lasers

A. Laser motifs

B. Synchronization of semiconductor laser rings

C. Sublattice synchronization 
D. Toward large laser networks

E. Stability of laser network synchronization

F. Beyond stability

G. Generalized synchronization and synchronization without correlation

V. Applications of Delay-Coupled Lasers

A. Laser source with tunable coherence length

B. Chaotic LIDAR and remote sensing applications

C. Random bit sequence generation

D. Chaos communication and chaos key distribution

E. From lasers to the brain

VI. Conclusions and Future Perspectives

A. Conclusions

B. Bioinspired photonic information processing

C. Toward integrated complex photonics

1. Integrated lasers with delayed feedback

2. Integrated coupled lasers and photonic integrated circuits

D. Final remarks

Acknowledgments

References

\section{INTRODUCTION: PAST AND VISION}

\section{A. Context: Semiconductor lasers}

The invention of semiconductor lasers, with the demonstration of lasing from semiconductor homojunctions in 1962, took place just two years after the demonstration of the first laser of any kind. Three groups managed to succeed within the same year (Hall et al., 1962; Nathan et al., 1962; Quist et al., 1962), which was scientifically and technologically a remarkable achievement. Nevertheless, semiconductor lasers remained laboratory curiosities for several years, because they needed to be operated at cryogenic temperatures and in pulsed operation due to a low external quantum efficiency. The breakthrough came with the introduction of heterostructures, a concept for which Kroemer and Alferov received the Nobel Prize in 2000 (Alferov, 2001; Kroemer, 2001). This breakthrough, together with major technological advances in semiconductor growth methods, enabled in the 1970s the growth of room-temperature, continuous-wave operated devices with operating lifetimes up to millions of hours.

Semiconductor lasers have come a long way since then. Nowadays, hundreds of millions of semiconductor lasers are every year being grown and built into a variety of systems. They are employed in optical storage systems, communication systems (ranging from short-distance data communication systems to long-haul fiber-optic networks), as pump sources, for material processing, and in many more applications; see, e.g., Agrawal (2010) and Bachmann, Loosen, and Poprawe (2010). They account for more than $50 \%$ of the laser market. Many varieties in terms of material composition, wavelengths, cavity geometries, gain concepts, array integration, and output powers exist, making them immensely versatile. The wavelengths of semiconductor lasers cover the range from the UV to the far infrared. Cavity geometries include edge and surface emitters, ring lasers, Fabry-Perot distributed feedback (DFB), and distributed Bragg reflector (DBR) structures. In recent years, photonic crystal structures, microcavities, and nanolasers have been pushing the development even further. Gain concepts comprise bulk semiconductor, quantum well, quantum dash and quantum dot structures, as well as the quantum cascade concept. Semiconductor lasers can be considered mature and ubiquitous devices and more varieties can be expected. Nevertheless, these lasers exhibit some particularities that need to be taken into consideration when using them in applications.

One particularity of semiconductor lasers is their extreme sensitivity to optical feedback and optical coupling. In this section, we review how this sensitivity, when first discovered, represented a nuisance. We then elaborate on how, from those first studies, semiconductor laser systems with delayed coupling have more and more been considered test-bed systems for the study of nonlinear dynamical systems with delayed coupling in general. Finally, we describe how, during the past decade, the potential of delay-coupled semiconductor lasers and their complex emission properties have been discovered for conventional and novel applications, ranging from encrypted communication, sensing applications, and complex networks to photonic information processing. These developments could contribute to the consolidation of a field we name complex photonics. Complex photonics targets the utilization of emerging behavior in networks and network motifs of (delay-) coupled nonlinear photonic systems. Here the term "complexity" refers to the importance of such emerging behavior, comprising nonlinear dynamics, synchronization, and other complex phenomena that occur in networks. Therefore, complex photonics does not refer to how complicated and extended the technical implementations are.

\section{B. Instabilities as a nuisance}

The study of instabilities in semiconductor lasers due to delayed coupling has a long history and has gone through different phases. As early as the late 1960s, instabilities in the emission were reported to appear when a semiconductor laser was coupled to an external cavity (Broom, 1969). At this time and throughout the 1970s, 1980s, and the early 1990s, a significant motivation to study these instabilities was to find ways to avoid or control them. It is worth mentioning though that already in 1970 Broom et al. noted that instabilities provide useful information on the fundamental physics of semiconductor lasers, and that the induced fast intensity modulation might even be useful for optical communication systems (Broom, Mohn, and Risch, 1970), a viewpoint that was again picked up later. But back then feedback-induced instabilities predominantly represented a nuisance to applications, and to some extent they still do. The reason is that the instability-induced intensity "noise" is much larger than the quantum noise in these lasers. Such enhanced intensity fluctuations were identified to have seriously deteriorating effects, particularly when they fell within the signal bandwidth, and therefore limited the usefulness of semiconductor lasers. In the low-frequency range, a dramatic increase in noise by as much as $60 \mathrm{~dB}$ induced by external feedback as small as $0.04 \%$ was reported (Miles et al., 1981). Therefore, instability phenomena due to delayed feedback have been a significant and fundamental problem for optical communication systems. Moreover, another major field of applications, 
optical data storage systems, was affected. The relative intensity noise (RIN) of semiconductor lasers used inside optical heads for reading and writing, usually sufficiently low, was identified to be enhanced because a small fraction of the laser output is invariably reflected from the disk surface back into the laser (Gray et al., 1993).

Different strategies have been followed to overcome or avoid these problems. On the one hand, it was attempted to make lasers less feedback sensitive, by working in regimes in which the enhanced fluctuations are weaker or outside the spectral range of interest. On the other hand, active disturbance or suppression of the instabilities in the emission of the lasers was investigated. A widely used active method for optical storage systems has been high-frequency injection, in which the laser current is modulated sinusoidally at frequencies much higher than the data rate. In 1983, Stubkjaer et al. first recognized that they could reduce the intensity noise by 15-20 dB when directly modulating the laser current (Stubkjaer and Small, 1983). The RIN increase was suppressed if the modulation frequency was suitably optimized, and if the modulation amplitude was sufficiently large such that the laser was pumped below threshold during a part of the modulation cycle (Gray et al., 1993). However, for this method the proper modulation frequency and amplitude had to be chosen empirically. For telecommunication applications, an optical isolator is usually included into the fiber-coupled laser modules, in order to avoid instabilities due to reflections from the optical fiber tip and from any type of back coupling. Optical isolators contribute to complexity and price of these modules, though. Finally, there has been hope within the past decade that with the use of modern gain concepts such as quantum dots, the nonlinearities in the lightsemiconductor interaction could be sufficiently reduced such that these lasers would be immune to delayed coupling instabilities. Although it was shown that indeed the sensitivity of quantum dot lasers to feedback instabilities can sometimes be lower, overall the hope for feedback insensitive semiconductor lasers was disappointed (Carroll et al., 2006).

\section{Semiconductor lasers as test beds for nonlinear dynamics studies}

During the 1980s, and even more in the 1990s, the study of semiconductor lasers became more and more motivated by their test-bed character for delay systems in general. Many characteristic nonlinear dynamical properties have been identified and thoroughly studied on semiconductor lasers. In fact, a whole field of laser dynamics has emerged, representing a prominent part in the nonlinear dynamics field. Laser systems allow for the study of the different routes to low- and highdimensional chaos, spatiotemporal dynamics, local and global bifurcations, multimode dynamics, chaos control, chaos synchronization, excitability, and stochastic resonance effects, to name only the most prominent aspects. Insights into this very active field can be found in several books; see, e.g., Weiss and Vilaseca (1991), Krauskopf and Lenstra (2000), Otsuka (2000), Erneux and Glorieux (2010), and Lüdge (2011). For the field of delay-coupled systems, lasers have played a catalyzing role. Many aspects of delay dynamics have been studied first in laser systems. A fruitful interaction with applied mathe- matics on delay systems has also evolved. But it is only now that different fields, in which delay-coupling plays a role, find and combine their concepts and insights. One example is the concept of zero-lag synchronization in delay-coupled systems that was first found in brain dynamics (Roelfsema et al., 1997). In delay-coupled semiconductor laser systems zero-lag synchronization was found and possible mechanisms identified based on delayed self-feedback (Klein, Gross, Rosenbluh et al., 2006) or dynamical relaying (Fischer et al., 2006). Subsequently, the mechanism based on a dynamical relay was transferred back to neuroscience, where it was demonstrated that it might play an important role in information integration in the brain (Vicente et al., 2008). Motivated by works on generic nonlinear oscillators and maps the role of local mechanisms for cluster synchronization in neural systems has also been reported (Kanter, Zigzag et al., 2011). From these aspects, semiconductor lasers with delayed coupling have emerged as important test-bed systems for delay-coupled networks. In return, the laser-focused studies benefit significantly from the general work on delay-coupled nonlinear oscillators and maps (Lakshmanan and Senthilkumar, 2010). Several concepts have relevance for or have been transferred to laser systems, e.g., the analysis of the stability of synchronization depending on the coupling topology, as discussed in Sec. IV. In order to keep the focus of this review, we restrict ourselves here to investigations which have a direct link to laser systems.

\section{Potential of complex photonics}

It is only recently that the study of delay-coupled semiconductor lasers has gained the maturity to aim for novel applications, including implementations of coupled network motifs or even larger networks. In 1993, the idea to use synchronization of two deterministic chaotic systems for encrypted communication was introduced by Cuomo, Oppenheim, and Strogatz (1993), working on electronic circuits, and Colet and Roy (1994) using solid-state lasers. Soon after, Mirasso, Colet, and Garcia-Fernandez (1996) recognized the potential to adapt the approach for semiconductor lasers with delayed feedback. The first experimental demonstration of the concept of communication using chaotic lasers came in 1998 from two groups (Goedgebuer, Larger, and Porte, 1998; Van Wiggeren and Roy, 1998). They showed that synchronization can be harnessed to implement encrypted communication based on deterministic chaotic carriers. The robustness and compatibility with optical communication networks was finally demonstrated in a field experiment in Athens in 2005 (Argyris et al., 2005). In that experiment, data rates of $1 \mathrm{Gbit} / \mathrm{s}$ with bit-error rates (BERs) down to $10^{-7}$ were achieved over $120 \mathrm{~km}$ transmission distance, using commercially installed optical fibers, applying forward error correction. In 2010, data rates up to $2.5 \mathrm{Gbit} / \mathrm{s}$ with error rates below $10^{-12}$ were achieved using deterministic chaotic devices, based on photonic integrated circuits (PIC) (Argyris, Grivas et al., 2010). In addition, Kanter, Kopelowitz, and Kinzel (2008) introduced a protocol for public channel cryptography. It is based on chaos synchronization of Bernoulli maps, but was tested in numerical simulations also for a model of delay-coupled semiconductor lasers. The synchronization could be preserved when 
the mutually transmitted signals were concealed by two commutative private filters. It was shown that then the task of the attacker can be mapped onto the class of NP-complete problems. The important conclusion from this is that semiconductor lasers with delayed coupling, although being unstable, can be sufficiently robust systems to be employed in applications, and even provide the potential for integration. It certainly requires a paradigm change to design inherently complex photonic systems instead of modularized and preferably linear systems, and to embrace those properties that for so long were considered a nuisance. Several other applications of the complex behavior of semiconductor lasers with delayed coupling have been identified. They comprise light detection and ranging (LIDAR), incoherent sources for rainbow refractometry, random number generation, and even photonic information processing. These applications will be described in more detail in Sec. V. They illustrate how versatile these systems are, with the flexibility to access different dynamical regimes and to accurately control their conditions. Still, the full capabilities of these systems will be exploited in the future. Complex behavior and its functional role is only now starting to be recognized and addressed in the brain, in population dynamics, or in social interactions. Cross fertilization among these different fields will inspire the design and the realization of complex photonics systems that might contribute to solving major issues in todays communication and information technology (IT) systems, including privacy, computational efficiency, or power consumption. Ultimately, the instabilities and complex behavior of semiconductor lasers with delayed coupling might turn from a curse into a blessing.

\section{DYNAMICS OF SEMICONDUCTOR LASERS WITH DELAYED SELF-COUPLING}

Semiconductor lasers with external cavities exhibit a variety of dynamical phenomena, depending on key parameters, comprising feedback strength, feedback delay, pump current, feedback type, and laser nonlinearity. The dynamics can be modeled and the origin of the instabilities understood using delay-differential equations. Until recently, detection and acquisition technology allowed one only to measure the dynamical phenomena with restricted resolution and with limited time series length. However, with novel technologies the full features can now be acquired and analyzed. In this section we review the dynamical phenomena of lasers with feedback, together with the theoretical approaches and the latest experimental techniques used to characterize these behaviors.

\section{A. Some history of lasers with delayed optical feedback}

Already in the early days of semiconductor lasers, it was recognized that the (semiconductor medium specific) ratio of electron lifetime $T_{e}$ and photon lifetime $T_{p}$ results in characteristic relaxation oscillations between the charge carrier reservoir and the photon reservoir. The relaxation oscillations are usually damped, and their frequency is typically of the order of $f_{\mathrm{RO}} \sim \mathrm{GHz}$. In 1969 and 1970, Broom et al. reported the first observation of intensity self-modulation at the

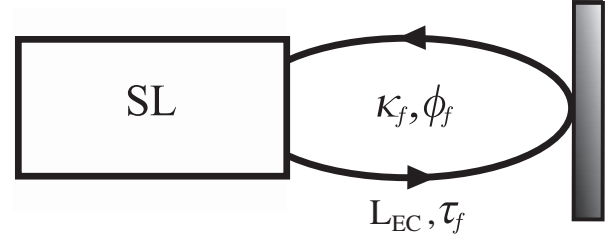

FIG. 1. Scheme of a semiconductor laser (SL) in an external cavity. The external cavity is characterized by the cavity length $L_{\mathrm{EC}}$, the corresponding round trip delay $\tau_{f}$, the feedback strength $\kappa_{f}$, and the feedback phase $\phi_{f}$.

relaxation oscillation frequency which was undamped via an external cavity, realized by a distant mirror (Broom, 1969; Broom, Mohn, and Risch, 1970). Figure 1 shows the scheme of a semiconductor laser in an external cavity, introduced by an external mirror. Broom et al. observed that the self-modulation was strongest when there was a resonance between $f_{\mathrm{RO}}$ and the round trip frequency in the external cavity $f_{\mathrm{EC}}=c / 2 L_{\mathrm{EC}}$, with $L_{\mathrm{EC}}$ being the length of the external cavity. Risch et al. (1977) observed that the light output of an external-cavity continuous-wave (cw) driven semiconductor laser self-pulsated at a resonance frequency of the external cavity and/or at a lower frequency in the range of 3-30 MHz. Since the low frequency is lower than any laser and cavity intrinsic frequency, the observed instabilities in the emission were named low-frequency fluctuations (LFF). Figure 2 depicts the intensity dynamics of the observed LFF for the lasing transverse electric (TE) and the much weaker transverse magnetic (TM) mode.

Together with the onset of instabilities, the occurrence of nonlinearities in the light output (power) versus injection current characteristic (L/I characteristic) has been observed. While in the beginning aging, degradation, and filamentation of the lasers were also identified as possible origins, Broom et al. suspected that the instabilities were no artifacts, but induced by the external cavity.

The ambivalent influence that feedback from an external mirror can have was already recognized in the 1970s. Bogatov et al. (1973) observed that, for the same laser, the optical feedback could give rise to a better selection of longitudinal modes and an increase in coherence in addition to the intensity self-pulsations at different parameters. This ambivalent influence created more interest in the phenomena induced by optical feedback and led to the development of

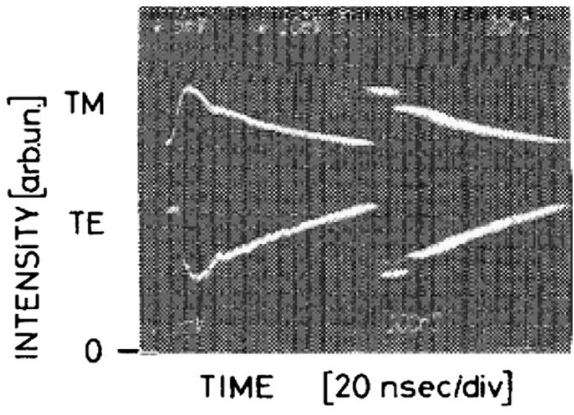

FIG. 2. Low-frequency intensity pulsations of a laser in an external cavity. From Risch et al., 1977. 
models to reproduce the static and dynamical properties. In 1980, Lang and Kobayashi published a rate equation model (the LK model) for a single-mode laser, describing the time evolution of the complex optical field and the carriers. They included the influence of the optical feedback by considering the interference of the laser field with its own coherent delayed field that had propagated once through the external cavity (Lang and Kobayashi, 1980). The model can be written as equations for the excess number of carriers $n(t)=$ $N(t)-N_{\text {th }}$ with respect to the solitary threshold level $N_{\text {th }}$, and for the slowly varying complex electrical field amplitude $E(t)$ :

$$
\begin{aligned}
& \dot{E}(t)=\frac{1}{2}(1+i \alpha) \xi n(t) E(t)+\kappa E\left(t-\tau_{f}\right) e^{-i \omega_{0} \tau_{f}}, \\
& \dot{n}(t)=(p-1) \frac{I_{\mathrm{th}}}{e}-\gamma_{e} n(t)-\left[\Gamma_{0}+\xi n(t)\right] P(t) .
\end{aligned}
$$

The optical feedback is taken into account via the feedback term, including $\kappa$ as the feedback rate and $\tau_{f}$ as the delay time. The optical field is normalized such that $P(t)=|E(t)|^{2}$ is the photon number, $\omega_{0}$ represents the angular optical frequency of the solitary laser, $\xi$ is the differential gain, $\Gamma_{0}=1 / T_{p}$ is the cavity decay rate, and $\gamma_{e}=1 / T_{e}$ is the inverse carrier lifetime. The bias current at the solitary laser threshold is denoted as $I_{\text {th }}, e$ is the electron charge, $p$ is the pump parameter, and $\alpha$ is the so-called linewidth enhancement factor discussed in more detail below. The excess phase $\phi_{f}$ that the optical field accumulates within the external cavity can be defined as $\phi_{f}=\omega_{0} \tau_{f} \bmod 2 \pi$.

Recognition of the importance of the delay in the feedback was crucial for further analysis and understanding of the observed phenomena. Consequently, Lang and Kobayashi induced a lot of activity on the subject, and the model turned out to be very successful.

Lenstra, Verbeek, and den Boef (1985) observed that the onset of instabilities in the emission of semiconductor lasers was linked to a reduction in coherence length by a factor of $1 / 1000$ due to optical feedback. They coined the name coherence collapse (CC) which has become a synonym for all instabilities in semiconductor lasers with delayed optical feedback. Two aspects turned out to be essential for the observation of the $\mathrm{CC}$ in semiconductor laser systems:

- the delay in the round trip of the light in the cavity and

- the nonlinearity in the interaction of the light with the semiconductor medium.

Since the existence and interplay of a (or multiple) time delay(s) and a nonlinearity are the key aspects in the behavior and understanding of delay-coupled systems in general, we discuss their particularities and consequences in the following.

\section{B. Consequences of delayed self-coupling}

Initial studies on the influence of delayed coupling (among few oscillators) and self-coupling (feedback on a single oscillator) on the dynamical behavior of oscillators were already undertaken in the 1960s in systems such as molecular oscillators (Marchenko and Rubanik, 1965) and electronic circuits (Marchenko, 1967). Back then, only the situations of small delays and weak nonlinearities could be tackled. However, the importance of delays for the onset of instabilities and synchronization of oscillators was already recognized. In control theory, the effect of delays was considered even earlier [see, e.g., Bellman and Danskin (1954)]. The first examples in optics for which the relevance of delayed feedback was identified are a model for a nonlinear ring cavity (Ikeda, 1979) and one year later the LK equations.

What makes delay systems so particular is the fact that their state space is infinite dimensional. The state space of a delay system with delay time $\tau$ is a space of continuous functions on the interval $[-\tau, 0]$. At any time $t_{0}$, the whole history of the system's variables within the interval $\left[t_{0}-\tau, t_{0}\right]$ is required to define the state of the system. The infinite-dimensional phase space and the resulting possibility to exhibit high-dimensional dynamics with many involved dynamical degrees of freedom illustrate the dramatic influence delays can have on dynamical systems. At the same time, exactly these features have been making delay systems so difficult to tackle from the mathematical side.

The fact that delays become relevant in semiconductor laser systems is closely linked to the fast light-matter interactions. The above-mentioned relaxation oscillations, which define a characteristic time scale for intensity pulsations of these lasers, exhibit typical periods of $T_{\mathrm{RO}}=1 / f_{\mathrm{RO}} \sim 100 \mathrm{ps}-1 \mathrm{~ns}$. This implies that even delays originating from short propagation lengths of light on the order of millimeter to centimeter cannot be neglected as compared to $T_{\mathrm{RO}}$. It is worth noting, however, that certain semiconductor lasers can exhibit relaxation oscillation periods outside of this range.

In many different contexts, delay-induced phenomena have been identified, and the types of systems in which delays are of importance include examples from as diverse areas as population dynamics, economics, autoimmune diseases (Mackey and Glass, 1977), neuroscience (Stepan, 2009), genetic oscillators (Elowitz and Leibler, 2000), and traffic models (Orosz, Wilson, and Szalai, 2009) or generic model systems such as phase oscillators (Yeung and Strogatz, 1999), to name only a few. Nevertheless, laser systems have boosted the studies of delay systems, complemented by the development of novel analytical (Erneux, 2009) and numerical tools (Engelborghs, Luzyanina, and Roose, 2002; Balachandran, Kamr-Nagy, and Gilsinn, 2009) for delaydifferential equations. Meanwhile, a multidisciplinary field on delay systems, their synchronization, and applications has developed (Just et al., 2010).

\section{Semiconductor laser nonlinearities}

Semiconductor lasers exhibit a particular nonlinearity in the interaction of the light with the active medium, which distinguishes them from all other lasers. The nonlinearity originates from the physics of the semiconductor band structure, since the photon generation typically occurs due to interband transitions. The gain spectrum of such lasers therefore does not exhibit a symmetric peak, as atomic transitions do, but has a strongly asymmetric shape. This affects the dispersive properties of the lasers as well, since those properties are connected via the Kramers-Kronig relation. As a consequence, the dispersion curve for the refractive index exhibits its zero crossing at higher frequency than the 
maximum of the gain spectrum. If the gain changes, e.g., by a change of the carrier density, the refractive index changes as well, which would not be the case for atomic transitions. Hence, changes in gain are in semiconductor lasers associated with changes in refractive index and vice versa. Since with a change of refractive index of the medium the optical frequency and thus the optical phase changes, one also speaks of amplitude phase coupling. A small change in the intensity (induced, e.g., by a change in the injection current, by dynamical instabilities, or even a spontaneous emission event) causes an excess perturbation of the phase of the lasing mode. In the rate equation description, the effect is taken into account via the so-called $\alpha$ parameter, which is also referred to as Henry parameter or linewidth enhancement factor. It is defined as

$$
\alpha=-\frac{d\left[\chi_{r}(n)\right] / d n}{d\left[\chi_{i}(n)\right] / d n},
$$

with $\chi_{r}$ and $\chi_{i}$ being the real and imaginary parts of the carrier-dependent susceptibility of the semiconductor material. This parameter was introduced by Henry (1982) to explain the broadening in the linewidth of semiconductor lasers, which is enhanced by a factor of $1+\alpha^{2}$ as compared to the Shawlow-Townes equation. It also accounts for many other effects such as frequency chirp, injection locking instabilities, and self-focusing. Besides the described mechanism originating from band filling, many-body Coulomb interactions also contribute to the $\alpha$ parameter. It is worth noting that the $\alpha$ parameter is not a constant for a given laser, but depends on parameters such as the spectral detuning of the laser emission from the gain maximum and also on the carrier density.

The amplitude phase coupling is not the only nonlinearity in semiconductor lasers. The occupation of the electrons in the conduction band can deviate from its Fermi-Dirac quasiequilibrium distribution due to carrier heating or spectral hole burning. These effects are beyond the rate equation description based on a two-level model. Nevertheless, the effect of this nonlinearity is often phenomenologically introduced into the rate equation model via a nonlinear gain saturation term of the form

$$
\xi=\xi_{0} /[1+\varepsilon P(t)]
$$

with $\varepsilon$ being called the nonlinear gain saturation or the $\varepsilon$ parameter. It has a rather damping effect on pulsations and the onset of instabilities. In addition to inherent laser nonlinearities, nonlinearities can be introduced via the coupling. This has, e.g., been utilized in optoelectronic feedback configurations that will be discussed in Sec. II.F.3.

In a simple form, we can hence describe a dynamical system with delayed coupling as

$$
\dot{x}(t)=f(x(t))+\kappa g\left(x\left(t-\tau_{f}\right)\right),
$$

where $\kappa$ is the feedback strength, and $f$ and $g$ can be either linear or nonlinear functions, possibly depending on further parameters. If $f$ is nonlinear, one says that the node (here the semiconductor laser) is nonlinear. If $g$ is nonlinear, it is the feedback which has nonlinearities. In the case of semiconductor lasers with optical feedback, $f$ is nonlinear and $g$ is linear. But in principle, all combinations are possible and with other coupling types nonlinear feedback or nonlinear coupling can also be introduced for semiconductor lasers.

\section{Phenomena and mechanisms of delayed optical feedback}

In the following, we focus on some dynamical features, representing complex behavior in semiconductor lasers with delayed optical feedback. This will mostly concern edgeemitting lasers, but other laser structures will be covered in the following sections as well. Several hundreds of papers have been published on the dynamics of this system. It would be impossible to provide a fair overview over the breadth and depth of the obtained insights. We therefore make a selection, highlighting those properties which, in our subjective view, might have a particular impact on the development of the field we denote as complex photonics. We will not go into the details of the theoretical derivations here, since excellent reviews on these aspects exist; see, e.g., van Tartwijk and Lenstra (1995) and Ohtsubo (2008).

We distinguish two main cases concerning the cavity length and therefore the feedback delays $\tau_{f}$ which induce the complex behavior. Depending on the ratio of the delay $\tau_{f}$ and the relaxation oscillation period $T_{\mathrm{RO}}$, we distinguish the qualitatively different long delay regime (LDR), where $\tau_{f} \gg$ $T_{\mathrm{RO}}$ and the short delay regime (SDR), where $\tau_{f} \lesssim T_{\mathrm{RO}}$. In these two regimes, the resulting dynamics exhibits conspicuous differences. It is important to note that in both regimes the delay is significant and determines the induced dynamics. Hence it cannot be neglected in either case. Both cases are also of practical relevance. The LDR is valid for most standard free-space and optical fiber-based configurations, and the SDR is of particular relevance for integrated structures.

\section{Long delay regime}

A major classification of the various effects of delayed feedback on semiconductor lasers was performed by Tkach and Chraplyvy (1986), on the particular case of a DFB laser emitting at a wavelength of $1.5 \mu \mathrm{m}$. The external cavity had a round trip length of $L_{\mathrm{EC}}=20 \mathrm{~cm}-4 \mathrm{~m}$. Tkach and Chraplyvy identified five different regimes of operation, increasing the amount of feedback from -80 to $-8 \mathrm{~dB}$.

Regime I is found for the lowest feedback levels of the order of $-80 \mathrm{~dB}$ feedback power ratio. Optical linewidth reduction and enhancement have been observed, depending on the feedback phase $\phi_{f}$. In regime II, at feedback levels depending on $L_{\mathrm{EC}}$, the linewidth enhancement gives rise to rapid mode hopping. The observed mode splitting depends on the feedback strength and on $L_{\mathrm{EC}}$. In the narrow regime III, observed around -45 to $-39 \mathrm{~dB}$ feedback, the mode hopping disappears, and the laser operates single mode. In regime $\mathrm{IV}$, starting from about $-40 \mathrm{~dB}$ feedback power ratio, relaxation oscillation sidebands appear. They grow for increasing feedback, and eventually the laser emission broadens up to $50 \mathrm{GHz}$ spectral width. This is the regime of CC. The feedback phase $\phi_{f}$ does not seem to affect the dynamical properties in this regime. Finally, in regime $\mathrm{V}$, for strong feedback greater than $-10 \mathrm{~dB}$, stable narrow linewidth emission has been observed, independent of $\phi_{f}$. 
These regimes provide a good overview of the possibly occurring phenomena, but feedback and the onset of certain regimes depend on the particular semiconductor laser under investigation. In the following, we concentrate on regime IV, extending over several orders of magnitude in feedback strengths, easily covering the range of -40 to $-10 \mathrm{~dB}$. It is the regime in which deterministic chaotic emission is frequently observed.

The first important step in understanding the complex emission dynamics was to analyze the structure of the fixed point solutions and their stability. Henry and Kazarinov (1986) analyzed the fixed point solutions of Eqs. (1) and (2). Without feedback, the system has, in the lasing regime, two fixed points: the stable lasing fixed point and the unstable nonlasing fixed point. The feedback induces many new rotating wave solutions with constant intensity $I$, constant frequency $\omega$, and constant inversion $n$. These solutions are relative equilibria with respect to the phase symmetry of the system. They were shown to lie on an ellipse in the space of $\Delta \omega \tau_{f}=\eta=\phi(t)-\phi\left(t-\tau_{f}\right)$ and $n$, with $\phi$ being the phase of the electric field. Figure 3 depicts such an ellipse. For long delays and moderate feedback levels, the number of induced fixed points is typically a few hundred and can even go into the thousands. Their number mainly depends on the feedback parameter $C=\kappa \tau_{f} \sqrt{1+\alpha^{2}}$ (Mørk, Tromborg, and Mark, 1992). For $C<1$ only one mode exists, for $C>1$ more modes exist, and for $C \gg 1$ the number of modes is approximately given by $C / \pi+1$. These fixed points can be subdivided into two classes: those belonging to a constructive interference condition between the light in the laser cavity and the feedback light (lower branch in the ellipse), and those belonging to a destructive interference condition (upper branch in the ellipse). In a physical interpretation, the former can be considered external cavity or compound cavity modes. The latter are correspondingly often denoted as antimodes and are saddle points (Mørk, Tromborg, and Mark, 1992).

For rather weak feedback of the order of $-40 \mathrm{~dB}$, the onset of instabilities for increasing feedback strength was studied in experiments by Mørk, Mark, and Tromborg (1990) and

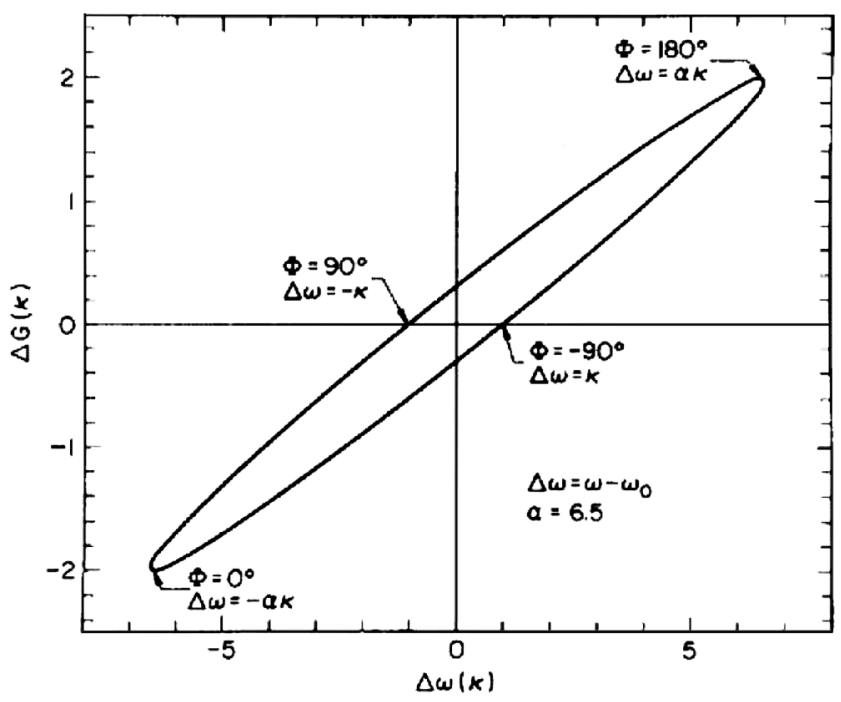

FIG. 3. Ellipse structure of fixed points in the $\Delta \omega \tau_{f}=\eta$ and $n$ space. From Henry and Kazarinov, 1986. theory by Tromborg and Mørk (1990). They showed via a stability analysis that the compound cavity modes are destabilized in a Hopf bifurcation via an undamping of the relaxation oscillations. This undamping initializes a route to chaos entering quasiperiodic and frequency-locked states, before deterministic chaotic behavior is reached. They could also analytically demonstrate that the instability threshold depends on the $\alpha$ parameter. The larger $\alpha$, i.e., the stronger the nonlinearity, the more sensitive semiconductor lasers are to delayed feedback. In the ellipse representation of Fig. 3, $\alpha$ determines the excentricity of the ellipse. In a similar regime of weak feedback, a route to deterministic chaos via perioddoubling bifurcations has also been identified ( $\mathrm{Ye}, \mathrm{Li}$, and McInerney, 1993). A detailed experimental and numerical study by Hohl and Gavrielides (1999) provided further insight into the sequence of routes to chaos, when the feedback is increased in small steps. It illustrates that, when varying the feedback strength or the feedback phase, modes and antimodes are always created in pairs via a saddle-node bifurcation, and they annihilate each other in pairs. In a further stability analysis, Levine et al. (1995) showed that the compound cavity mode with the largest gain, the so-called maximum gain mode, always remains stable; however, it can coexist with other attractors and might not always be reached. A comprehensive review of stability and feedback-induced noise properties for weak feedback can be found in Petermann (1995).

For intermediate feedback levels, LFF and fully developed $\mathrm{CC}$ behaviors dominate. After their first observation by Risch et al. (1977), the investigation of LFF and CC regimes was significantly advanced in the late 1980s and the 1990s. Much insight into the characteristics of these phenomena and the involved physical mechanisms was gained. Mørk, Tromborg, and Christiansen (1988) recognized in numerical simulations that the LFF might coexist with other attractors and may look more like in the experiments when a significant amount of noise was added into the field equation. Figure 4 depicts such numerically obtained LFF with the noise term switched off at $t=100 \mathrm{~ns}$. Without noise the laser exhibits quite strong pulsations, while with the addition of sufficiently strong spontaneous emission noise, the characteristic slow envelope of the LFF with the sudden power drops and somewhat slower recovery can be seen. The onset of the strong pulsations without noise was speculated to be an indication for

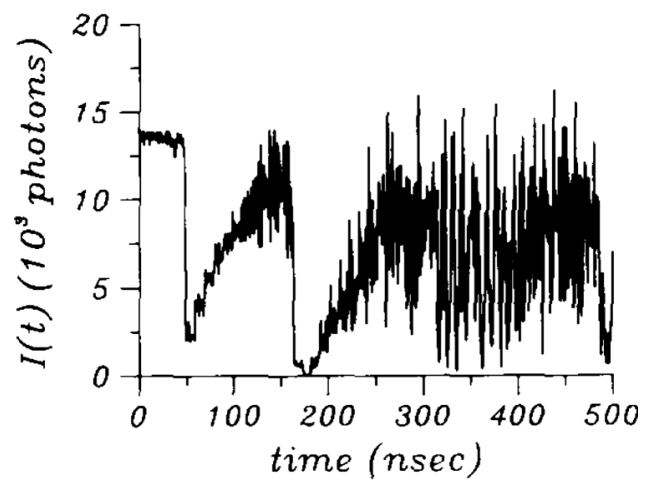

FIG. 4. Numerically obtained LFF for moderate feedback, $p=2$, $\tau_{f}=10 \mathrm{~ns}$, and strong field noise. For $t>100 \mathrm{~ns}$ the noise has been switched off. From Mørk, Tromborg, and Christiansen, 1988. 
deterministic chaotic dynamics. It was Sano (1994) who recognized the importance of the short pulsations for the understanding of the mechanism leading to the LFF. He interpreted the dynamics as a chaotic itinerancy process (Ikeda, Otsuka, and Matsumoto, 1989) toward the compound cavity mode with the largest gain. The chaotic itinerancy is linked to the emission of fast chaotic pulsations. The dropout then occurs due to a crisis, such that the trajectory gets attracted by a stable manifold of one of the antimodes and then is rejected back into the low gain region, with the consequence that the power drops and a new cycle is started. The fast pulsations could subsequently be experimentally demonstrated (Fischer, van Tartwijk et al., 1996). Figure 5 depicts the experimentally obtained LFF dynamics, recorded with an oscilloscope, back then restricted to $1 \mathrm{GHz}$ resolution bandwidth, and the fast irregular pulsations, measured with a streak camera with 8 ps temporal resolution.

A fruitful and controversial debate developed in order to clarify whether the chaotic itinerancy process or other mechanisms explain the LFF behavior best. In particular, the role of multilongitudinal mode emission and the role of noise have been investigated in detail and will be discussed in Secs. II.E and II.G. For single-mode lasers with not too strong feedback, the deterministic mechanism, as described by Sano (1994), turned out to be rather successful, and good agreement was found between various aspects in experiments and the LK model; see, e.g., Heil et al. (1999) and Liu, Davis, and Takiguchi (1999). Still it needs to be emphasized that several approximations included in the derivation of the LK model (e.g., the assumption of a two-level homogeneously broadened gain medium, single-mode behavior, weak to moderate feedback, and the phenomenological description of amplitude phase coupling) can fail under particular experimental conditions and by the choice of laser.

Besides the studies of the mechanism of LFF, the parameter regimes in which LFF occur depending on pump parame-
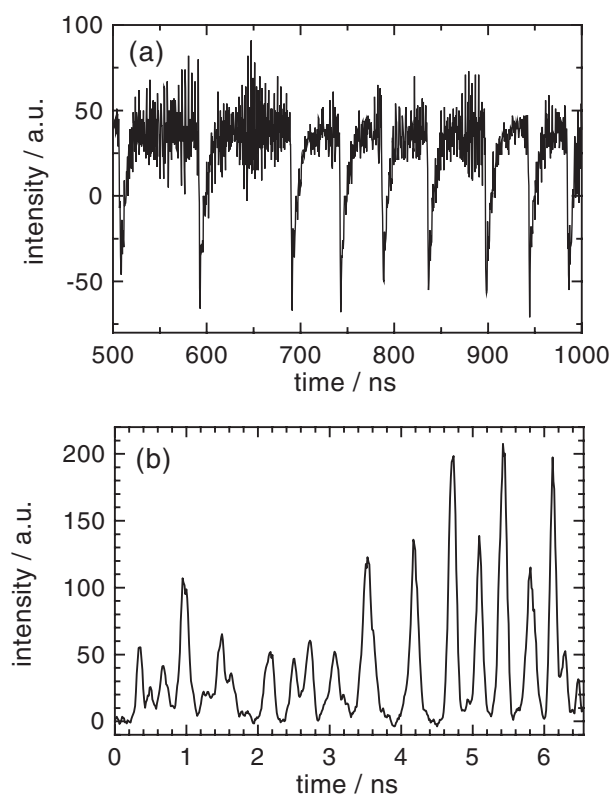

FIG. 5. Experimentally obtained LFF for moderate feedback, $p=2.03$, and $\tau_{f}=3.6 \mathrm{~ns}$. (a) Oscilloscope time trace, and (b) streak camera trace. From Fischer, van Tartwijk et al., 1996. ter $p$, feedback rate $\kappa$, and nonlinearity $\alpha$ have been studied and characterized in detail (Heil, Fischer, and Elsäßer, 1999). This particularly refers to the transitions to fully developed $\mathrm{CC}$ for increasing current and to the coexistence of LFF and stable emission on the maximum gain mode (or a mode close to it) for rather strong feedback and small $\alpha$. The mechanism for the $\mathrm{CC}$ is, within the LK model description, similar to the one of the LFF. The main difference is that the antimodes at which the trajectories are rejected back into the lower gain region are closer to the solitary laser frequency and spectrally more spread. As a consequence, the low-frequency features disappear from the observed intensity dynamics.

Most of the experiments were restricted to the measurement of the optical output intensity, but the carrier dynamics was measured as well (Ries and Sporleder, 1982; Ray et al., 2006). This allows one to access two of three physical quantities of the system described by the LK equations (1) and (2). Figure 6 depicts the experimentally obtained probability distribution functions of LFF events for both quantities, measured with a bandwidth of $1 \mathrm{GHz}$. The obtained results are consistent with the predictions of the LK model.

In recent years, significant insight has been gained into the nature of the delay instabilities in the LK equations, the fundamental bifurcations, and the properties and topology of the emerging chaotic attractor. The development of new mathematical tools for the treatment of delay-differential equations has played an important role. They allow one to address the instabilities in delay systems with a combination of numerical and analytical methods, which was deemed very hard for a long time. Numerical simulations alone proved to
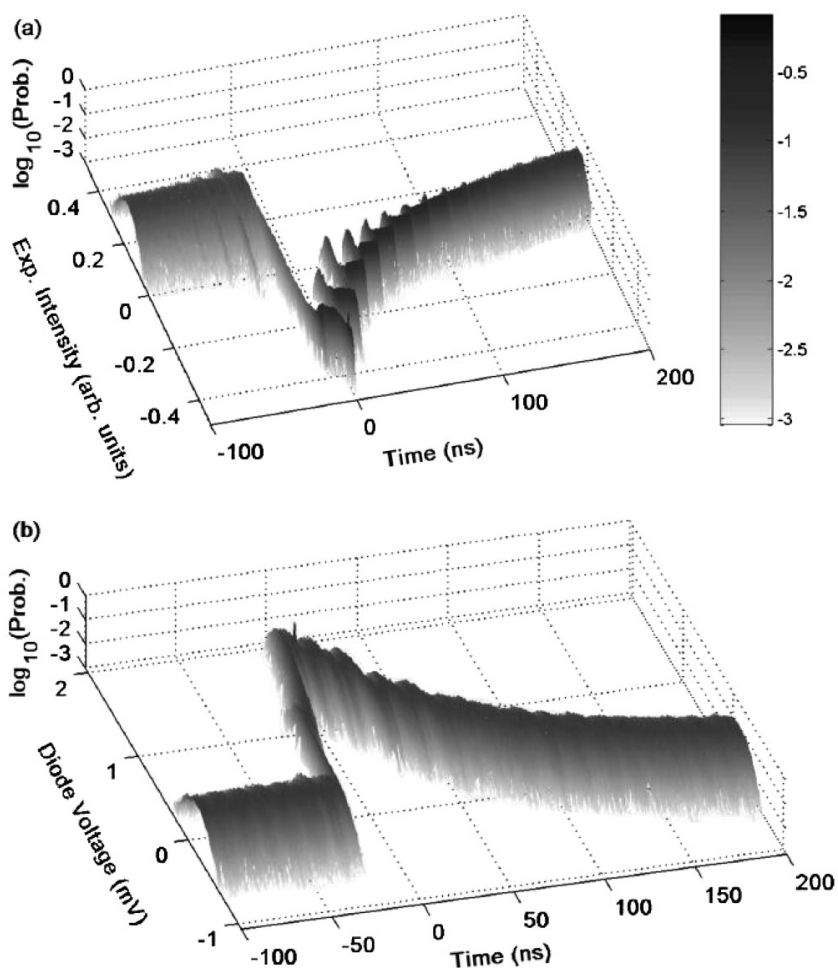

FIG. 6. Experimentally probability distribution functions of LFF events for (a) intensity dynamics and (b) carrier dynamics. Parameters were $p=2.01$ and $\tau_{f}=14.3 \mathrm{~ns}$. From Ray et al., 2006. 
be valuable, but have restrictions for the understanding of underlying mechanisms and are also limited due to the multidimensional parameter space. Based on a detailed bifurcation analysis, Wolfrum (2002) identified an organizing center for the appearance of more complicated dynamics. Particularly, continuation methods were made accessible for delay systems (Engelborghs, Luzyanina, and Roose, 2002) and allowed for an insightful analysis of the delayed-feedback laser and to identify how different bifurcations connect. Haegeman et al. (2002) used numerical continuation methods exploiting the symmetry properties of the LK equations. They showed that pairs of modes and antimodes are connected by closed branches of periodic solutions (Haegeman et al., 2002). Furthermore, separation of time scales has proven useful in order to treat delay systems analytically (Erneux, 2009).

In recent years, the understanding of the topology and nature of the chaotic attractor of delay systems in the long delay limit has improved significantly. In a series of papers, Yanchuk and collaborators identified generic properties of systems with time delay, which are related to the appearance and stability of periodic solutions (Wolfrum and Yanchuk, 2006; Yanchuk, Wolfrum et al., 2006; Yanchuk and Perlikowski, 2009). They showed that delay systems generically have reappearing families of periodic solutions that for increasing delay overlap. This leads to an increased coexistence of solutions. In addition, they showed that the spectrum of characteristic multipliers can be split into two parts: pseudocontinuous and strongly unstable, in which the pseudocontinuous part of the spectrum mediates destabilization of periodic solutions. Heiligenthal et al. (2011) identified two different types of delay instabilities related to strong and weak chaos, distinguished by the scaling properties of the maximum Lyapunov exponent. For the LK model, they found a scenario leading from weak to strong chaos and back to weak chaos with increasing feedback strength. Weak chaos is related to the analogy of delay systems with spatially extended systems. In that case the delay is interpreted as a "spatial" size of a one-dimensional system, compatible with the observation that the dimensionality of the attractor increases linearly with the delay time $\tau_{f}$ (which is the system size) (Le Berre et al., 1987; Giacomelli and Politi, 1996; Bünner et al., 2000).

The importance of instabilities due to delayed (self-) coupling with long delay is underlined by investigations on lasers with different cavity geometries and modern gain concepts, such as vertical-cavity surface-emitting lasers (VCSEL), quantum dash and quantum dot lasers, as well as quantum dot microlasers. The theoretical description might in some cases require different or adapted modeling to account for a second polarization mode or for the different gain concepts, but the observed phenomena are similar. Delayed-feedback instabilities have also been found and characterized in detail for VCSEL; see, e.g., Jiang, Dagenais, and Morgan (1995), Fujiwara, Takiguchi, and Ohtsubo (2003), Sondermann and Ackemann (2005), and Tabaka et al. (2006)). Although VCSEL were initially speculated to be less sensitive to external reflections due to their high reflecting mirrors, it turned out that their sensitivity to delayed feedback is similar to edge-emitting lasers, since their photon lifetimes $T_{p}$ are comparable. VCSEL usually have small gain differences for the two orthogonally polarized laser modes. Therefore, unstable behavior is often associated with polarization dynamics. Polarization mode instabilities can even be deliberately introduced by polarization-rotated feedback, as discussed in Sec. II.F.2.

From the viewpoint of laser instabilities as a nuisance, much hope was put into quantum dot lasers. This hope was raised by the fact that an individual quantum dot has, similar to atomic transitions, a symmetric gain peak. Therefore, simple considerations, as described in Sec. II.C, would result in very low values for $\alpha$. Experimental measurements, however, yield strongly varying results, depending on the used method, the laser structure, and operating conditions (Melnik, Huyet, and Uskov, 2006). This can be understood by not only considering the carriers in the quantum dots themselves, but also those in the so-called wetting layer, surrounding the quantum dots. Consequently, delayed-feedback instabilities have also been experimentally found in quantum dot lasers (Carroll et al., 2006). Quantum dot lasers are, however, less sensitive as compared to quantum well lasers, which partly originate from their higher relaxation oscillation damping rate. Quantum dash lasers, being structurally intermediate to quantum wells and quantum dots, have been found to exhibit similar LFF and CC behavior to quantum well lasers (Azouigui et al., 2007). Recently, quantum dot microlasers have also been investigated. Albert et al. (2011) demonstrated that the fingerprints of deterministic chaos can even be observed for such lasers operating close to the quantum limit at nano-Watt output powers. A dramatic change in the photon statistics was observed. Albert et al. expected that this might open up new perspectives for the study of chaos in quantum systems.

Beyond the described systems and phenomena above, other regimes of optical feedback and different feedback schemes have attracted considerable attention. Some of them are briefly presented in the following.

\section{Short delay regime}

The short delay regime was already studied by Bogatov et al. (1973) and Lang and Kobayashi (1980) and peaks related to induced pulsations could be identified in the intensity power spectrum. It was recognized early that the phase of the feedback light $\phi_{f}$ strongly determined the impact on the emission of the laser, in contrast to most dynamical regimes in the long delay case. Tager and Petermann (1994) derived a condition that described the critical feedback strength for the onset of instabilities and the frequencies of self-oscillations for weak feedback. From an application point of view, they considered their result a guideline for the design of high-speed laser diodes with an integrated passive cavity. A temporal resolution of the deterministic chaotic instabilities, however, became possible only with the development of fast real-time oscilloscopes or by using a streak camera. It was Heil, Fischer, Elsäßer, and Gavrielides (2001) who first studied the short delay instabilities in semiconductor lasers in experiments with high time resolution. Striking dynamical phenomenon were identified in this regime with the help of a streak camera: the emission of regular and irregular pulse packages defined by a 
low-frequency envelope with underlying fast intensity pulsations. From numerical modeling it could be concluded that the dynamics takes place on the same ellipse of compound cavity modes and antimodes as described for the long delay case, however with much fewer modes involved. A feedback phase dependent scenario from stable emission via irregular pulse packages and periodic pulse packages back to stable emission was demonstrated (Heil, Fischer et al., 2003). A numerical bifurcation analysis, in combination with an analysis of the unstable manifolds, revealed the nature of the underlying global bifurcations.

While the experiments by Heil, Fischer, Elsäßer, and Gavrielides (2001) were based on a solitary laser and a distant mirror, Ushakov et al. (2004) studied integrated devices. In these devices, the delayed feedback originates from an integrated passive section of only $200 \mu \mathrm{m}$ length. Still, it turned out, that delayed feedback-induced self-pulsations can be observed in these devices. In addition, even amplified feedback schemes can be implemented (Bauer et al., 2004). For such devices, phase and strength of the feedback can be separately tuned. Different kinds of self-pulsations, eventually reaching chaotic behavior, were observed. It is worth noting that time-delayed feedback has also been used to stabilize continuous-wave emission (Schikora et al., 2006; Flunkert and Schöll, 2007; Dahms, Hövel, and Schöll, 2008). Subsequently, monolithic integrated laser structures based on delayed feedback were designed to exhibit chaotic emission. Such lasers were realized and characterized by Argyris et al. (2008). They consisted of a distributed feedback laser, a passive resonator, and active elements that control the optical feedback properties. It was demonstrated that stable cw solutions, periodic states, and broadband chaotic dynamics could be accessed, by tuning feedback phase and pump conditions. Figure 7 depicts such a scenario for variation of the electric current, controlling the feedback phase. From the spectra, compound cavity mode peaks can be seen in Figs. 7(a) and 7(e), while chaotic coherence collapse dynamics is obtained in Figs. 7(c) and 7(d). Such integrated structures show the potential for the realization of compact and robust devices. In fact, these devices have already been employed for encrypted communication purposes as detailed in Sec. V.D.

\section{E. Multimode effects}

\section{Longitudinal modes}

In the early investigations of delayed-feedback instabilities, the studied lasers often had a Fabry-Perot cavity defined by their cleaved facets. Therefore, they could exhibit emission in several longitudinal laser modes, while most of the modeling was performed using the single-mode LK equations. In the mid 1990s the question arose what the role of these additional longitudinal modes is, when and in how far they modify the mechanism of the instabilities, and whether they are even essential for the observed instabilities in the coherence collapse regime. Different regimes were identified in which the multiple modes have a significant influence on the chaotic pulsation statistics or have only little influence (Huyet, 1998; Sukow et al., 1999). Vaschenko et al. (1998) measured the irregular pulsations in the contributing modes
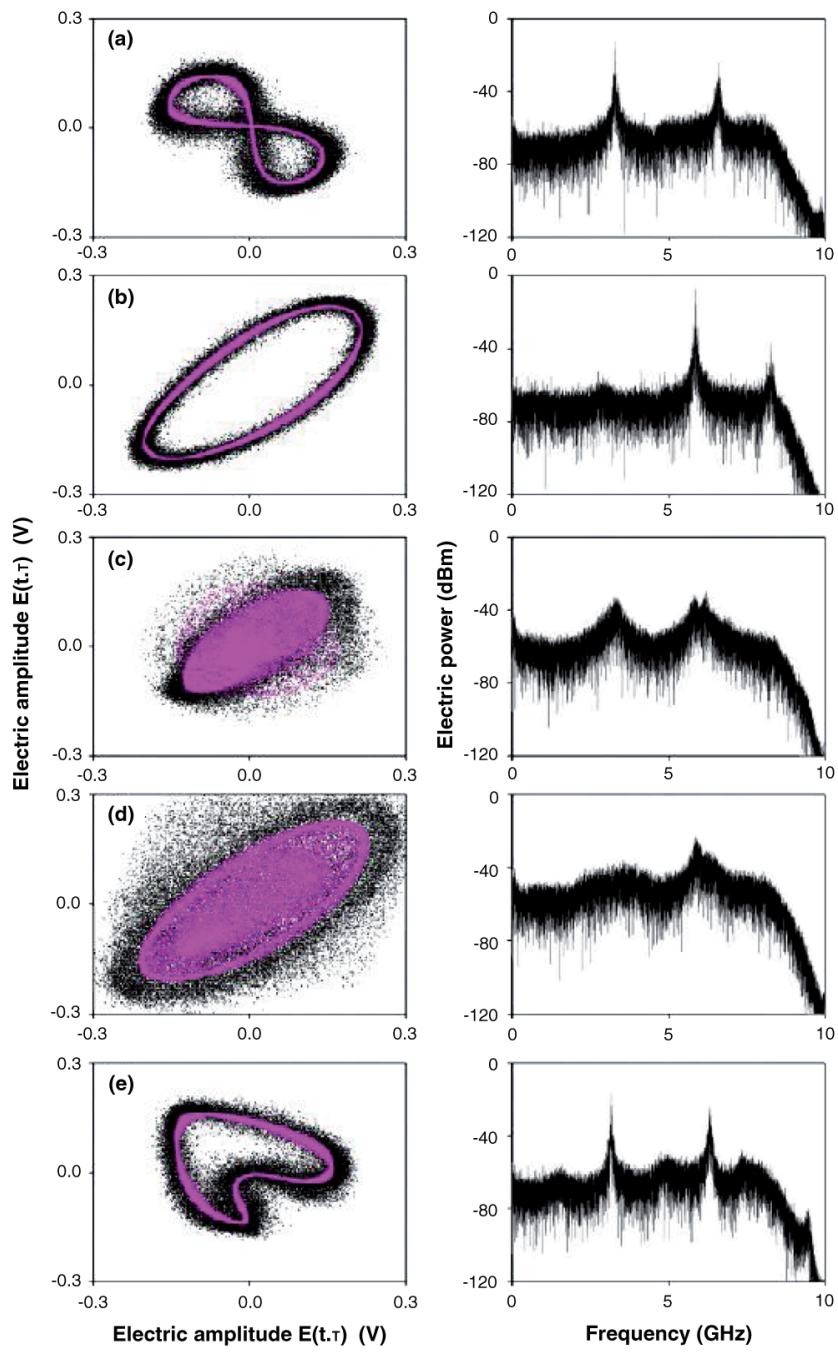

FIG. 7 (color online). Experimentally obtained phase dependent scenario for a monolithic integrated laser structure. Experimental phase plots (left column) and corresponding intensity power spectra (right column) of the device output for varying the phase current (a)-(e), $I_{\mathrm{ph}}=3.3,4.8,5.5,5.9$, and $6.9 \mathrm{~mA}$, respectively. From Argyris et al., 2008.

with a streak camera. To describe the multimode dynamics adequately in modeling, various multimode extensions to the LK model were proposed, introducing a separate equation for each mode (Sukow et al., 1999; Viktorov and Mandel, 2000). It was finally clarified that multimode behavior can modify the deterministic chaotic dynamics and even the underlying mechanism, but that often the single-mode description is sufficient (Huyet et al., 1999; Sukow et al., 1999; Viktorov and Mandel, 2000). Concerning the relation among the modes, Uchida et al. (2001) experimentally showed that the oscillations around the relaxation oscillation frequency were in phase for all the modes, while the oscillations at lower frequencies exhibited partial antiphase behavior.

Peil, Fischer, and Elsäßer (2006) studied multimode dynamics in the extreme case in which the short external cavity was coupling the longitudinal modes of the laser resonantly. For such strong modal coupling, distinct differences to the short-cavity regime of nonresonant laser systems were reported. The most striking difference was the onset of chaotic 
broadband dynamics comprising more than 100 lasing longitudinal modes. A thorough understanding of such dynamics is still lacking; nevertheless it can be utilized in applications as described in Sec. V.A. Tanguy et al. (2006) extended the experimental studies to multimode quantum dot semiconductor lasers. They found a highly organized chaotic antiphase dynamics of the various modes, leading to a constant total output power. Although, multimode behavior represents a challenge for theoretical analysis, it offers interesting dynamical properties, which may be utilized in applications.

At this point, it is important to mention that more detailed modeling of semiconductor lasers can be performed, taking a more semiconductorlike description of the medium susceptibility into account (Balle, 1998), or even modeling the microscopic properties of the semiconductor active medium; see, e.g., Hess and Kuhn (1996), Chow, Sargent, and Koch (1997), and Moloney et al. (1999). These models play an important role to adequately describe particular semiconductor materials and their ultrafast carrier dynamics and are very successful if predictions of laser properties for a specific gain material are needed. Because of their complexity, they are, however, difficult to employ for the study of detailed parameter dependencies of delayed-feedback systems or even analytic treatment such as stability analysis. Still they represent a valuable option when device specific modeling is required.

\section{Transverse modes: Spatiotemporal instabilities}

Semiconductor lasers with large apertures can exhibit spatial effects and complex spatiotemporal dynamics in their emission even when operated solitarily. For quasicontinuous wave operation and even when pumped with constant current such phenomena have been predicted and observed. This applies to broad area edge-emitting lasers (Hess, Koch, and Moloney, 1995; Fischer, Hess et al., 1996; Tanguy et al., 2004), multistripe devices (DeFreez et al., 1988; Winful and Rahman, 1990; Hess and Schöll, 1994; Merbach et al., 1995), and broad area VCSEL (Hegarty, Huyet, and McInerney, 1999; Barchanski et al., 2003). Still those lasers are sensitive to delayed feedback as well. Broad area edge emitters have been found to exhibit pulse packages and coherence collapse phenomena when they are subjected to additional optical feedback from a short external cavity (Mandre, Fischer, and Elsäßer, 2005; Tachikawa et al., 2010). The ambivalent nature of delayed feedback can sometimes even be employed to stabilize or suppress the spatiotemporal instabilities. Several studies implemented control techniques to suppress filamentation or spatiotemporal emission by utilizing delayed optical feedback (Martín-Regalado et al., 1996; Mandre, Fischer, and Elsässer, 2003; Wolff and Rodionov, 2003; Chi, Thestrup, and Petersen, 2005). Although simple, optical feedback methods improved the beam profile, the spectral width, and the power stability of these lasers.

\section{F. Other feedback schemes}

Besides the presented feedback scheme by a distant mirror, several other schemes have been considered and their influence on the dynamics of semiconductor lasers has been investigated. Here only a brief overview of these other schemes is given, although they too allow one to realize a variety of dynamical phenomena that can be of interest for applications in the complex photonics field. A detailed overview of other feedback schemes is provided by Ohtsubo (2008).

\section{Frequency-selective feedback}

Frequency-selective feedback represents a flexible and efficient solution to reduce the optical linewidth or to tune the emission wavelength of semiconductor lasers. In fact, nowadays, tunable laser sources incorporating external cavities formed by reflective optical gratings represent important devices and find applications, e.g., in laser cooling and spectroscopy. Semiconductor lasers with feedback from a reference cavity can be stabilized to have optical linewidths which can go down to below $1 \mathrm{~Hz}$ (Kolachevsky et al., 2011). Nevertheless, frequency-selective feedback has also been found to induce instabilities depending on the feedback strength, spectral selectivity, and tuning. This was already recognized by Risch and Voumard (1977). From a dynamics point of view, the effects of frequency-selective feedback were addressed, modeling optical feedback from a FabryPerot resonator (Yousefi and Lenstra, 1999), from a grating (Detoma, Tromborg, and Montrosset, 2005), and from a fiber Bragg grating (Naumenko, Besnard, and Loiko, 2003). The frequency selectivity modifies the mode distribution of the system and affects their stability. Modified and different dynamical effects can emerge. In experiments, the impact of the relation between filter width, relaxation oscillation frequency, and external-cavity frequency on the dynamical behavior was studied; see, e.g., Fischer et al. (2004) and Erzgräber et al. (2006). A particularly interesting feature of filtered coherent optical feedback is the onset of complex dynamics, including frequency oscillations, for which the intensity remains almost constant. In summary, frequencyselective feedback provides an opportunity to tune and modify the dynamical properties induced by delayed feedback and adds to the versatility of the system.

\section{Polarization-rotated feedback}

Optical feedback provides the freedom to manipulate the polarization of the feedback light. In this way it allows one to modify the interaction of the fed back light with the optical field in the laser and to involve other polarization modes in the emission. Loh, Ozeki, and Tang (1990) introduced a quarter-wave plate into the external cavity of an edgeemitting semiconductor laser, such that the feedback light polarization was rotated by $90^{\circ}$ with respect to the emitted light. As a result, the laser exhibited square-wave-like polarization self-modulation with a period of twice the delay time $\tau_{f}$, and for high pump currents showed a transition to chaotic emission. Li, Hohl, and Gavrielides (1998) extended these results to experiments with VCSEL, in which a similar phenomenology was found. For high-frequency selfmodulation in the $\mathrm{GHz}$ range, the modulation characteristics became, however, more and more sinusoidal.

If the polarization mode gain difference is too large, or the feedback not sufficiently strong, the weaker polarization mode might not be excited. Still instabilities can emerge due to the interaction of the feedback light with the carrier reservoir in the laser (Otsuka and Chern, 1991). This situation 
has often been referred to as incoherent feedback. Since it is a priori not clear whether only one or both polarization modes are involved in the dynamical processes, we prefer to speak, in general, of polarization-rotated optical feedback. Heil, Uchida et al. (2003) studied a laser with large polarization mode gain difference and could still identify weakly chaotic dynamics, different from the scenario observed by Loh, Ozeki, and Tang (1990). Heil, Uchida et al. (2003) showed that an incorporation of the weak TM mode was required to model the dynamical behavior. The full flexibility of these setups was recently explored by Khaykovich et al. (2009) and Gavrielides et al. (2010), considering further polarization mode coupling schemes. Gavrielides et al. (2010) studied a configuration allowing for the strong TE mode to couple to the weak TM mode, but not vice versa. They found an extension of the previously simple square waves to more complex variants with different shapes and periods. Khaykovich et al. (2009) studied TE-TM coupled mode dynamics in a semiconductor laser subject to feedback with variably rotated polarization, finding dynamical instabilities in a large parameter range.

\section{Optoelectronic feedback}

Besides optical feedback schemes, optoelectronic feedback schemes have also found a lot of interest for the study of complex dynamics. They benefit from the possibility that the pump current of semiconductor lasers can be directly modulated with $\mathrm{GHz}$ bandwidths. In such schemes, the intensity of the laser is detected at a particular point, and the amplified electrical signal is fed back to the pump current of the semiconductor laser. The detector might directly measure the emitted laser intensity, or the light might have passed beforehand through an optical configuration. In this way, different kinds of feedback nonlinearities can be designed and studied. The delayed feedback to the injection current can again induce instabilities in the emission of the semiconductor laser systems. Deterministic chaotic behavior has indeed been observed in various configurations. We mention only a few examples of these intensely studied systems here. Liu and Ohtsubo (1991) studied such systems in the early 1990s, employing a laser Twyman-Green interferometer with delayed electronic feedback. A period-doubling route and successive higher harmonic bifurcations to chaos were observed in this system, showing similarities to a delay scenario as described by Ikeda and Matsumoto (1987). Takizawa, Liu, and Ohtsubo (1994) employed a Fabry-Perot interferometer whose output was detected and fed back to the laser current, also finding period-three cycles and chaos. Goedgebuer, Larger, and Porte (1998) introduced an optoelectronic wavelength oscillator. It consists of an electrically tunable DBR multielectrode laser with a feedback loop formed by a delay line and an optical configuration which exhibits a nonlinearity in wavelength. The light is detected after transmission through a birefringent plate and polarizers, and fed back to the tuning section of the laser. This system exhibits chaotic dynamics in the wavelength of the laser, again in close correspondence to the scenario as described by Ikeda and Matsumoto (1987). Figure 8 depicts experimentally obtained bifurcation diagrams for two different optical path differences introduced by the birefringent plate.

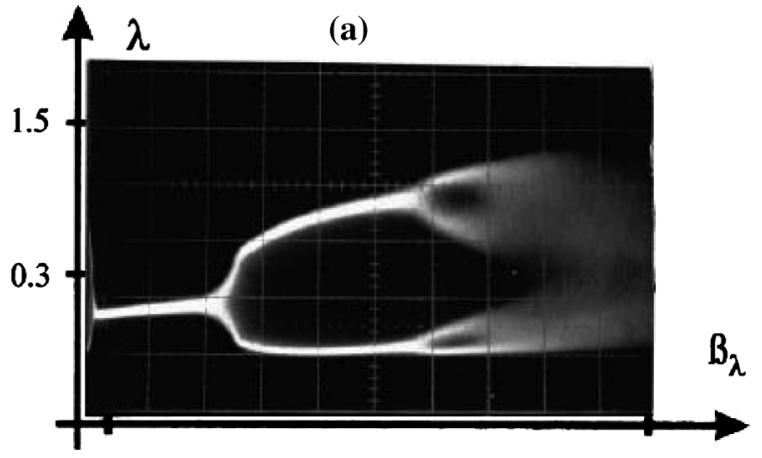

(b)

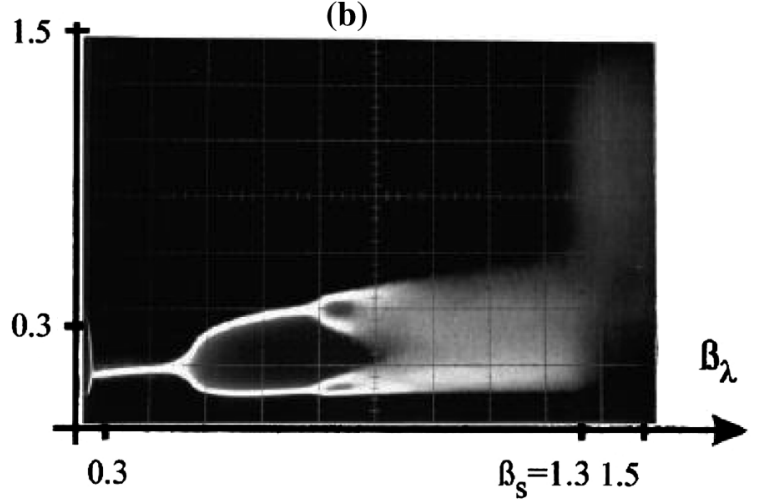

FIG. 8. Experimental bifurcation diagrams for optoelectronic feedback scheme showing the transition to wavelength chaos in dependence on the feedback strength $\beta_{\lambda}$. From Goedgebuer, Larger, Porte, and Delorme, 1998.

Even the systems with direct detection of the laser's output and delayed electronic feedback to the pump exhibit a rich variety of complex behavior. Tang and Liu (2001a) studied the system with positive feedback, and Lin and Liu (2003) studied the system with negative feedback to the pump current. Many variations of these feedback schemes have been studied intensively since then and employed for applications as well as for investigation of fundamental properties of delay-coupled systems, as discussed in the following sections. The importance of optoelectronic chaotic systems based on delayed feedback has recently been highlighted by Larger and Dudley (2010).

\section{Phase-conjugate feedback}

The study of the effect of phase-conjugate feedback on semiconductor lasers has also found considerable interest. Most of these studies focused on the stabilization of lasers and to reduce their linewidth, but some studies concentrated on dynamical effects as well. In such studies, it is important to take the characteristic time scales of the phase-conjugating medium into account. In modeling, instantaneous (van Tartwijk, van der Linden, and Lenstra, 1992; Murakami, Ohtsubo, and Liu, 1997; Krauskopf, Gray, and Lenstra, 1998) and noninstantaneous (Green and Krauskopf, 2004) phase-conjugate feedback have been considered. From the experimental side, three different kinds of phase-conjugating mirrors have been employed: photorefractive crystals such as $\mathrm{BaTiO}_{3}$, atomic vapors, and broad area semiconductor lasers. $\mathrm{BaTiO}_{3}$ crystals allow for high reflectivities but have very 
slow response times on time scales of seconds (Feinberg, 1982). Using atomic vapors in a four-wave mixing configuration, high reflectivities with response times down to the nanosecond scale can be achieved. Broad area semiconductor lasers offer high reflectivities and fast time response times reaching picosecond time scales (Kürz, Nagar, and Mukai, 1996). With a focus on dynamical properties, experimental results were reported using rubidium vapor and $\mathrm{BaTiO}_{3}$ crystals. For the rubidium vapor mirror, feedback power levels up to $-26 \mathrm{~dB}$ were achieved. Different frequency locking as well as coherence collapse phenomena with a period-doubling route to chaos was identified (Andersen et al., 1999). For feedback from a $\mathrm{BaTiO}_{3}$ crystal, Lawrence and Kane (2001) compared phase-conjugate and conventional optical feedback in a system using the same laser device in the long delay regime. For phase-conjugate feedback they found coherence collapsed, chaotic behavior covering optical feedback levels which were even larger than in the case of conventional mirror feedback.

The large variety of feedback schemes, only briefly presented here, illustrates the flexibility and versatility of semiconductor laser systems for delay-coupled networks.

\section{G. Influence of noise}

Noise in semiconductor lasers has two major contributions: field noise (originating mainly from spontaneous emission) and carrier noise (which has its origin in the carrier recombination and is, in addition, affected by the noise of the pump source). In theoretical models, they are usually accounted for by inclusion of Langevin noise terms $F_{E}(t)$ in the field equation (1) and $F_{n}(t)$ in the carrier equation (2). The field noise is often considered to affect the dynamics more strongly, but the role of the carrier noise has been addressed in detail as well. The influence of noise has two aspects. First, it can affect the dynamical states and their stability. Second, noise might even play a potentially constructive role, giving rise to ordering effects. Therefore, with a perspective on applications, noise will be an important factor to consider. The two aspects of noise robustness and a possible ordering role of noise are discussed in the following.

\section{Noise robustness}

The aspect of noise robustness is best explained on the concrete example of the LFF phenomenon. A long-lasting and important discussion has been whether the LFF are a noise-induced phenomenon, a noise-modified phenomenon, or a deterministically dominated effect. Prior to the deterministic mechanism as described by Sano (1994), Henry and Kazarinov (1986) had introduced an explanation for the LFF, describing it as a noise-induced escape phenomenon from one of the stable high-gain modes. Hohl, van der Linden, and Roy (1995) studied the LFF phenomenon in numerics and experiments and analyzed the statistics of the power dropouts. They found that, in some parameter regimes, spontaneous emission noise qualitatively influences the statistics of the dropouts in agreement with the predicted dependencies of Henry and Kazarinov (1986). This finding seems to particularly hold for pump currents very close to threshold, where the influence of spontaneous emission is maximal. However, it was not clear whether in this case one of the stable high-gain modes was reached prior to a dropout event. Later, for a laser with a small $\alpha$ parameter, a stable mode was shown to be reached before a power dropout was induced (Heil, Fischer, and Elsäßer, 2000). Under those conditions, the distribution functions of the residence times, as described by Henry and Kazarinov (1986), were reproduced. For added carrier noise, a similar scaling law for the noise dependence of the residence times was demonstrated. In particular, the noiseinduced escape from the basin of attraction of the stable mode was shown to exhibit similarities to the classical problem of thermally induced escape from a potential well. This further stimulated the discussion whether the LFF are a persistent dynamics or rather a chaotic transient. In numerical modeling of the LK equations, it was observed that, for small values of the $\alpha$ parameter, the system always ended up in a stable mode, which could be prevented by adding a sufficient amount of noise (Torcini et al., 2006; Zamora-Munt et al., 2010). For larger values of $\alpha$, even without noise terms, the LK model shows persistent LFF dynamics. The determination of the relevant $\alpha$ parameter from experiments is, however, not an easy task. Different methods of parameter characterization exist, but often the conditions under which $\alpha$ is obtained do not correspond to the conditions under which the instabilities are observed, and significant error bars exist. Highly resolved optical spectra might, in the near future, allow for a more meaningful determination of $\alpha$.

The influence of carrier noise on the complex behavior of semiconductor lasers was for a long time neglected, because the optical linewidth of the stable emission of a semiconductor laser is determined by phase fluctuations originating from spontaneous emission noise and is not affected by the carrier noise (Henry, 1982). However, Yousefi, Lenstra, and Vemuri (2004) recognized that carrier noise can affect the dynamical state of a semiconductor laser subject to optical feedback. Van der Sande et al. (2006) showed that low-frequency noise added to the injection current of a semiconductor laser can decrease the relaxation oscillation frequency $f_{\mathrm{RO}}$ and increase its damping rate. Moreover, current noise can also suppress the instabilities significantly, as demonstrated by Soriano, Berkvens et al. (2011). In the latter work, current noise was added to the pump current in the low-frequency domain, and a damping of the spectral peaks corresponding to the intensity instabilities up to $10 \mathrm{~dB}$ was observed, as long as the feedback was not too strong. For moderate to strong feedback, no influence of the current noise on the CC dynamics was noticed.

\section{Ordering role of noise}

As indicated by the latter example, the notion of order in nonlinear systems is not trivially related to the amount of noise in the system, as it is for linear systems. In fact, it is well known that a certain amount of noise can increase the order in the dynamics of a nonlinear system. Two examples of the ordering role of noise are the amplification of the response of nonlinear systems to weak external forcing, known as stochastic resonance (Gammaitoni et al., 1998), and the generation and enhancement of periodic behavior in nonlinear systems close to rhythm-generating bifurcations, denoted as coherence resonance (Gang et al., 1993; Pikovsky and 
Kurths, 1997). Both types of phenomena have been identified in the LFF regime of a semiconductor laser with delayed optical feedback. Marino et al. (2002) performed an experiment in which they applied a sinusoidal current modulation to a semiconductor laser with optical feedback, in addition to broadband current noise that could be varied in amplitude. They found that, for a finite external noise level, there is a frequency for which the LFF occur almost periodically at the modulation frequency. This resonant frequency matched the inverse of the average interdropout time. For a fixed forcing frequency the same resonance was found by varying the noise level, a well-known characteristic of stochastic resonance. This type of behavior has also been reproduced by simulations of the Lang-Kobayashi model (Buldú et al., 2002), which revealed a nontrivial dependence of the phenomenon with respect to the feedback time (Buldú, García-Ojalvo, and Torrent, 2004). Masoller (2002) extended this concept to multistable behavior in model calculations. She studied a similar configuration with weak feedback for which several attractors coexist and for which large enough noise induces jumps among the attractors. She showed that for a certain noise level the dynamics of attractor jumps exhibits a resonant behavior due to the interplay of noise and delayed feedback.

Giacomelli et al. (2000) demonstrated the existence of coherence resonance in the delayed-feedback semiconductor laser system. Changing the amount of noise, they showed that a resonance existed at which the LFF dropouts were most regular. Buldú et al. (2001) performed modeling on this system based on the LK equations with external colored noise in the pump current. An optimal coherent response was found for suitable values of both the amplitude and the correlation time of the noise. Martinez Avila et al. (2004) subsequently demonstrated in experiments and modeling that coherence resonance exists in this system even without external noise drive. Fast deterministic dynamics was shown to play the role of an effective exciting noise source. Coherence resonance was also reported in other semiconductor laser configurations (Panajotov et al., 2004; Ushakov et al., 2005). In a subsequent development, numerical simulations and experimental results revealed the existence of the phenomenon known as ghost stochastic resonance, where the laser responds to a complex external signal (consisting of harmonics of a missing fundamental) by detecting the ghost fundamental frequency, as long as an optimal amount of noise (in this case provided by the internal dynamics of the laser) is present (Buldú et al., 2003; Van der Sande et al., 2005).

The above discussed influences of noise illustrate that interesting phenomena might appear due to the interplay of noise and delayed feedback. They need to be considered in coupling configurations as well, where they can in addition affect synchronization properties, as discussed in Sec. III.F. Therefore, noise effects should be taken into account for any experimental implementation and might even be used in a constructive way.

\section{H. Advances and challenges in acquisition and characterization of semiconductor laser dynamics}

The phenomena of delayed feedback in semiconductor lasers have been studied in great detail in the past and represent the basis for the study of delay-coupled semiconductor laser systems. Nevertheless, advances in photonic sources and components, as well as novel and improved acquisition technologies, open up new perspectives for their study and utilization. The implementation of semiconductor laser systems with delayed coupling employing fiber optics allows one to utilize off-the-shelf telecommunication system components which are mature and low cost. They permit one to scale the setups to large delays and more complicated configurations. They even allow one to realize network configurations that are otherwise hard to adjust and control. Some network motifs will be addressed in Sec. IV.A. With the development of multichannel fast real-time oscilloscopes, one of the major obstacles in studying the delay dynamics of semiconductor lasers has been overcome. Recently, realtime oscilloscopes with an analog bandwidth beyond $10 \mathrm{GHz}$, reaching up to $45 \mathrm{GHz}$, additionally providing memory depths of tens of millions of samples per acquisition, have become available. In combination with fast photodetectors, that are standard technology in telecommunications, long time series of the intensity dynamics covering the full bandwidth of delayed-feedback instabilities can be acquired for the first time. This will finally enable a more detailed time series analysis of experimental data from these systems. Figure 9 shows a comparison of dynamics in the LFF regime, in the upper panel for $1 \mathrm{GHz}$ bandwidth limited detection, and for $16 \mathrm{GHz}$ bandwidth detection using modern acquisition techniques in the lower panel, illustrating the amount of gained dynamical resolution. These advances are complemented by the possibility to measure not only the intensity dynamics, but also the carrier dynamics of the lasers (Ray et al., 2006). Even the frequency dynamics can now

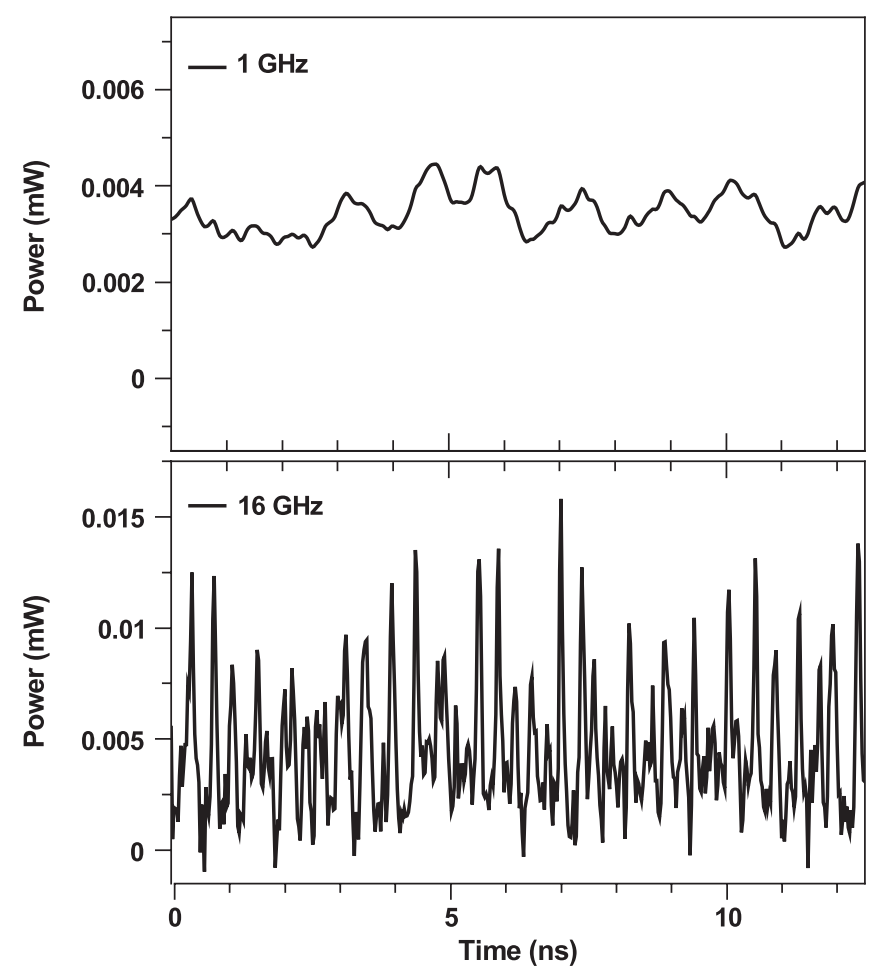

FIG. 9. Comparison of LFF dynamics, detected with $1 \mathrm{GHz}$ bandwidth (upper panel) vs $16 \mathrm{GHz}$ bandwidth detection (lower panel). Courtesy of Daniel Brunner, IFISC, Spain. 
be measured with nanosecond resolution, using heterodyne techniques (Brunner, Porte et al., 2012). In addition, the heterodyne technique, as well as novel methods in optical spectrum analysis, based on nonlinear mixing in fibers, allows one to resolve the compound cavity mode separation, and at the same time to cover the whole spectral width, which can extend over 4 to 5 orders of magnitude. Altogether new characterization methods have become possible, giving rise to unprecedented insights into the dynamical phenomena and processes. Moreover, they also allow one to determine the laser and external-cavity parameters and experimental conditions with higher precision. This will enable a better comparison of modeling and experiments and to target dynamical phenomena more precisely.

\section{DYNAMICS AND SYNCHRONIZATION OF DELAY-COUPLED SEMICONDUCTOR LASERS}

The study of coupled lasers started soon after the laser discovery. Monolithically integrated coupled-cavity devices were considered for applications such as optical bistability (Lasher, 1964) or amplification (Kosonocky and Cornely, 1968). Cleaved-coupled-cavity lasers, also called $C^{3}$ lasers, were proposed in the early 1980s. $\mathrm{C}^{3}$ lasers can be built as an active-passive (laser-mirror) or active-active (laser-laser) system. Soon after, the development of high power lasers led to the study of the dynamical properties of arrays of semiconductor lasers coupled via their evanescent fields (Botez and Ackey, 1986; Wang and Winful, 1988; Botez and Mawst, 1996). While the output of the individual elements of the array can be dynamically unstable, exhibiting large amplitude chaotic pulsations, the total output turned out to be characterized by a quasisteady state with small amplitude fluctuations (Wang and Winful, 1988). Winful and Rahman (1990) were the first numerically showing that a subset of lasers in an array of coupled lasers can produce identical, synchronized, chaotic signals for a certain coupling regime. Outside that range, synchronization breaks down, and the system enters a regime of spatiotemporal chaos or turbulence. Because of the intrinsic fabrication process of the devices described before, the delay in the propagation time of the light between the elements is very small compared to the intrinsic time scale $\left(T_{\mathrm{RO}}\right)$ of the system and can, consequently, be neglected.

This picture drastically changes when two or more lasers are coupled such that the propagation time for the optical signal from one laser to the others is of the order of, or larger than, the characteristic time scale of the lasers. In semiconductor lasers, with characteristic time scales $T_{\mathrm{RO}}$ of hundreds of picoseconds to nanoseconds, this already occurs for separation distances of some millimeters to centimeters; we refer to these systems as delay-coupled lasers. Delay coupling introduces additional degrees of freedom into the problem. Mathematically, the system becomes infinite dimensional, as described in Sec. II, and a rich variety of behaviors can be expected (Erneux, 2009), including multistability of synchronized and desynchronized states, amplitude death in coupled limit cycle oscillators, or stochastic, coherence, or ghost resonance or chaos suppression, for instance, in networks of oscillators with random delays. One of the pioneering studies of delay-coupled systems was reported in 1989, considering limit-cycle oscillators that mutually entrained each other (Schuster and Wagner, 1989). Since then, the dynamics of the delay-coupled systems has become a fascinating and exciting field that covers not only fundamental studies, but also a variety of applications, some of which will be presented in this review.

In this section we present an overview of some of the different behaviors that can be observed when coupling two or more semiconductor lasers with a certain delay. We begin with the simple case of unidirectionally coupled lasers, which can exhibit identical or generalized synchronization phenomena, depending on the laser parameters and operating conditions. Next we address the case of bidirectionally coupled lasers, for which the different types of synchronization states are linked to different relative timing of the individual emissions. In two bidirectionally coupled lasers a spontaneous symmetry breaking occurs under certain conditions that prevents the emergence of isochronous identical synchronization. This limitation can be overcome by introducing self-feedback to the lasers, an external drive laser, or a relay element that can be a third laser or a semitransparent mirror.

\section{A. Identical synchronization, generalized synchronization, and consistency}

Dynamical systems, in general, and semiconductor lasers, in particular, can synchronize their dynamics when properly coupled. This ability plays an important role in some applications, the most relevant being chaos-based communications (see Sec. V.D). Synchronization was first defined as the ability of two systems to adjust their rhythms in time when they are weakly coupled (Pikovsky, Rosemblum, and Kurths, 2001). However, from practical considerations, this definition needs to be extended to include, e.g., strong coupling and entrainment, the latter referring to the case in which one dynamical system drives the dynamics of another system. In this review, we follow this broader notion of synchronization that is widely accepted in the scientific community.

From the many types of synchronization that have been identified in coupled systems, identical synchronization, phase synchronization, lag synchronization, generalized synchronization, etc. (Boccaletti et al., 2002), we concentrate on two types: identical synchronization, sometimes also referred to as complete synchronization, and generalized synchronization. Identical synchronization is the simplest form of synchronization in which two (almost) identical systems, operating in a periodic or even chaotic regime, perfectly hook each other, and exhibit identical oscillations in time. Given two systems described by the state vectors $\vec{x}(t)$ and $\vec{y}(t)$, identical or complete synchronization implies $\vec{x}(t)=$ $\vec{y}(t) \forall t$. Generalized synchronization goes further in considering different systems and associating the output of one system to a given function, often unknown, of the other system. In order to define generalized synchronization more precisely, let us consider the unidirectionally coupled system of dimension $n$ and $m$, respectively:

$$
\dot{\vec{x}}=F(\vec{x}), \quad \dot{\vec{y}}=G(\vec{y}, h(\vec{x})),
$$


where $F: R^{n} \rightarrow R^{n}, G: R^{m} \rightarrow R^{m}$, and $h(\vec{x}(t)): R^{n} \rightarrow R^{m}$. Then the two systems are said to be synchronized in a generalized sense if a transformation $\psi$ exists which is able to asymptotically map the trajectories of system $\vec{x}$ onto system $\vec{y}$, regardless of the initial conditions, such that $\vec{y}(t)=$ $\psi(\vec{x}(t))$. While identical synchronization can be identified by, e.g., calculating the cross-correlation function between two signals, generalized synchronization is more difficult to test. A simple and appealing way (from a practical point of view) to test generalized synchronization is by using the auxiliary system approach introduced by Abarbanel, Rulkov, and Sushchik (1996). Assuming, for instance, that one deals with a drive-response system, this approach requires the use of a copy of the response system, the auxiliary system [see Fig. 10(a)], which is driven by exactly the same input as the original response system, although starting from different initial conditions. By observing a stable regime of identical, or quasi-identical, oscillations in the auxiliary and response systems, it is said that the drive and response systems are synchronized in generalized form. How far the auxiliary system approach corresponds to the precise definition of generalized synchronization is yet under investigation. Besides the concept of generalized synchronization, consistency was also defined (Uchida, McAllister, and Roy, 2004). Consistency refers to the ability of a nonlinear system to respond identically, or almost identically, to the repetitive application of the same complex drive signal, while starting from different initial conditions [see Fig. 10(b)]. If identical or almost identical responses are obtained repetitively, it is said that the nonlinear system responds consistently. This concept is important since it evaluates the capacity of a dynamical system to produce the same, or almost the same, output under the same input. In principle, it is also less demanding to check experimentally when compared to generalized synchronization, because it does not require an auxiliary system. To what extent generalized synchronization, the auxiliary system approach, and consistency refer to the same concept is still under debate.
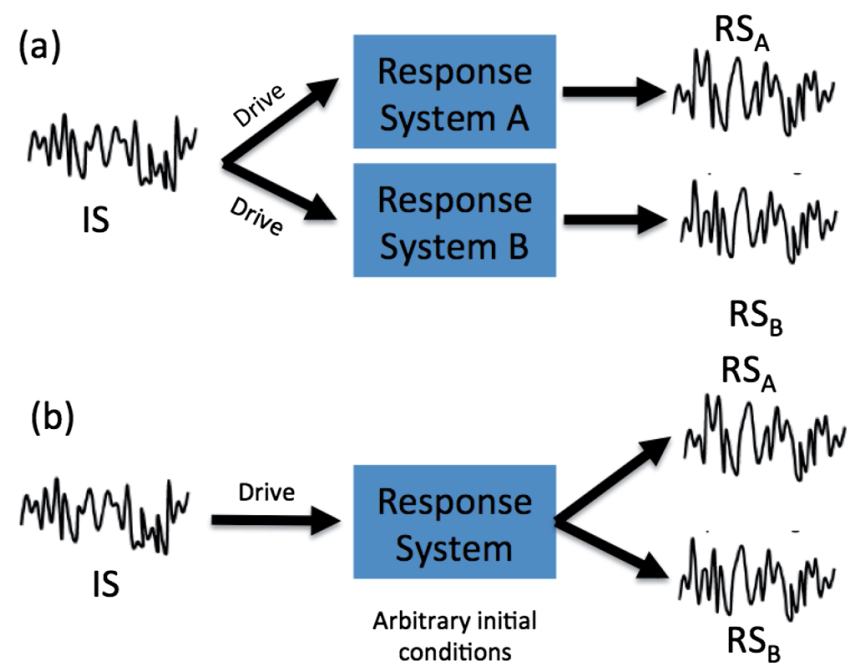

FIG. 10 (color online). Schematic representation of the (a) auxiliary system approach to detect generalized synchronization, and (b) consistency test.

\section{B. Unidirectional coupling}

Synchronization properties of coupled lasers have been extensively studied from both fundamental and applied viewpoints. One of the simplest configurations considers a laser, usually called a drive laser (DL), injecting part of its light into another laser, usually called a response laser (RL) in a unidirectional way. If the DL operates in $\mathrm{cw}$ or in a periodic regime, while the RL operates in cw when uncoupled, the RL can, for instance, be locked in frequency to the DL (stable injection locking) or operate in a periodic, quasiperiodic, or even chaotic regime, depending on the coupling strength and the optical frequency detuning between both DL and RL (van Tartwijk and Lenstra, 1995; Locquet, Masoller, and Mirasso, 2002; Murakami and Ohtsubo, 2002). If the DL operates in a chaotic regime, that could be induced for instance by an external mirror or by an optoelectronic self-feedback as mentioned in Sec. II, the RL can synchronize its dynamics to the DL either identically or in a generalized way. As an example, let us assume that the DL is subject to delayed optical feedback with strength $\kappa$ and feedback delay $\tau_{f}$ (see Fig. 11) such that it operates in a chaotic regime. Also assume that the RL operates in the $\mathrm{cw}$ regime when uncoupled and in the absence of any feedback (open-loop scheme, when the dashed box is removed in Fig. 11). If the DL and RL are optically coupled with a coupling strength $\sigma$, identical synchronization can be achieved if $\kappa=\sigma$ holds and the two lasers operate with a negligible frequency detuning (Ahlers, Parlitz, and Lauterborn, 1998). In the upper panel of Fig. 12 the electric field amplitude of the DL $\left[E_{0}(t)\right]$ is plotted versus time, in the middle panel the electric field amplitude of RL $\left[\tilde{E}_{0}(t)\right]$, and in the lower panel their normalized difference $\Delta E_{0}(t)=\left|\tilde{E}_{0}(t)-E_{0}(t-2 \Delta t)\right| /\left\langle E_{0}(t)\right\rangle$. Because of the coupling delay $\tau_{c}$, the intensity signal $\tilde{E}_{0}(t)$ of the response laser is shifted in time with respect to the drive (Ahlers, Parlitz, and Lauterborn, 1998). In the lower panel it can be seen that after a short transient the two output signals become perfectly synchronized.

The detuning between emitting frequencies of the two lasers plays a crucial role for identical synchronization. Even a small frequency detuning prevents the lasers from synchronizing, as seen in Fig. 13, where experimental results of the correlation function versus the detuning frequency between DL and RL are plotted for the case in which the self-feedback power of the DL equals the injected power into the RL (Liu et al., 2002). In this unidirectional coupling configuration, the coupling delay $\tau_{c}$ between the DL and the RL, i.e., the propagation time of the light between DL and RL, does not play any role in the synchronization process; it only shifts the position of the peak where the maximum of the cross-correlation function occurs. In the particular situation in which the coupling time is shorter than the feedback time in the DL $\left(\tau_{c}<\tau_{f}\right)$, the maximum of the cross-correlation

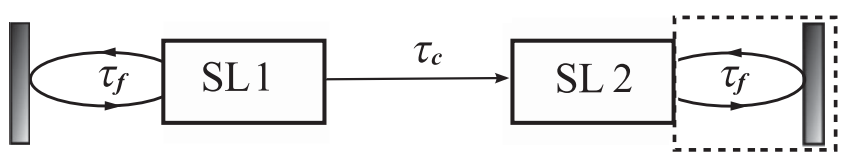

FIG. 11. Scheme of a setup for the unidirectionally coupled laser system. 


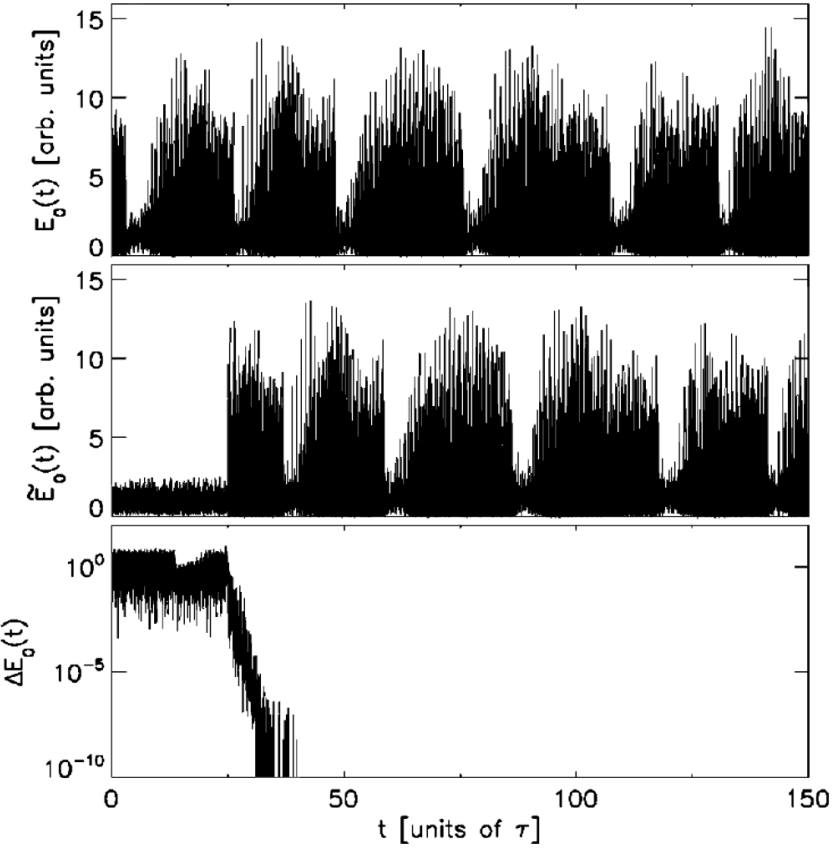

FIG. 12. Low-frequency intensity pulsations of a laser in an external cavity. From Ahlers, Parlitz, and Lauterborn, 1998.

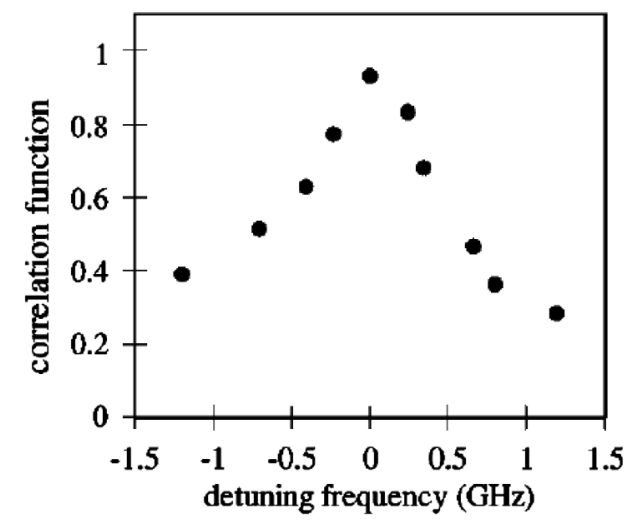

FIG. 13. Experimental results of the correlation function vs the detuning frequency. From Liu et al., 2002.

function is located at times shorter than $\tau_{c}$ and the observed synchronization is also referred to as anticipated synchronization (Masoller, 2001; Liu et al., 2002). However, the condition $\tau_{c}<\tau_{f}$ is not necessary for anticipated synchronization to occur. Actually, for $\tau_{c}>\tau_{f}$ the RL still synchronizes to the future state of the ML. If the RL laser is also subject to the same kind of optical feedback as the DL (closed-loop scheme, with the dashed box in Fig. 11), with a feedback strength $\kappa^{\prime}$ and feedback delay $\tau_{f}$, the trajectory of the two coupled lasers undergoes different bifurcations (Hopf, period doubling, torus, and crisis) for increasing coupling strengths. This gives rise to diverse dynamical regimes (periodic, quasiperiodic, and chaotic and bistability), eventually reaching very high correlation for sufficiently large coupling (Ruiz-Oliveras and Pisarchik, 2009). Identical synchronization can occur if $\kappa=\sigma+\kappa^{\prime}$. Identical synchronization is also possible for certain values of $\kappa<\sigma+\kappa^{\prime}$ due to interference (Flunkert and Schöll, 2012) but otherwise, generalized synchronization or no synchronization is observed.

The stability of the identically synchronized solution can be analyzed and determined in terms of different models. One of the most used models is based on modified LangKobayashi equations (Lang and Kobayashi, 1980) described in Sec. II, including a term that couples the DL with the RL (Revuelta et al., 2002). In the latter work, a simple theory is developed to obtain synchronization conditions and to analyze the effects of the detuning between the two lasers.

\section{Dynamics and synchronization of two bidirectionally coupled semiconductor lasers}

When two semiconductor lasers are placed in a face-toface configuration with a coupling delay that is of the order of or longer than the characteristic time scale of the laser $\left(T_{\mathrm{RO}}\right)$, interesting and sometimes unexpected phenomena can be observed, depending on the operating conditions. In the short delay regime (where the coupling delay is of the order of $T_{\mathrm{RO}}$ ) it was found both in experiments and in numerical modeling that, by detuning the optical frequency between the two lasers, delay-induced scenarios ranging from optical frequency locking to successive states of periodic intensity pulsations emerge (Wünsche et al., 2005). Interestingly, the theoretical treatment revealed the universal character of these findings for delay-coupled systems in general (Wünsche et al., 2005). In the following, however, we concentrate on the long delay limit, for which the coupling delay is larger than the relaxation oscillation period $T_{\mathrm{RO}}$ of the lasers.

One of the first studies in the long delay regime considered two weakly coupled semiconductor lasers (Hohl et al., 1997). Specifically, they investigated a system of two coupled lasers placed at a distance of $20 \mathrm{~cm}$ where the two lasers were pumped at different levels. Under such asymmetric conditions the lasers have different free-running relaxation oscillating frequencies and different intensities. It was found that the laser which was pumped at the lower level could entrain the laser that was pumped at a considerably higher level. In this context, synchronization refers to the locking of the relaxation oscillation frequencies of the individual lasers. This localized synchronization was characterized by low amplitude oscillations in one laser, in conjunction with large oscillations in the other laser. In Fig. 14 experimental optical spectra of the two semiconductor lasers are depicted, demonstrating the localized synchronization state (Hohl et al., 1997).

When the coupling strength is increased, the situation radically changes. In particular, it was found both experimentally and numerically that when two identical semiconductor lasers (with respect to internal parameters and operating conditions) are placed far apart and coupled face to face via their emitted electric fields, subnanosecond, couplinginduced synchronized chaotic dynamics in conjunction with a spontaneous symmetry breaking can occur (Fujino and Ohtsubo, 2001; Heil, Fischer, Elsäßer, Mulet, and Mirasso, 2001; Mulet et al., 2004). The chaotic oscillations are accompanied by a leader-laggard dynamics in which the leader-laggard role of the two lasers changes randomly with time; over a long time window, both lasers lead or lag, on 


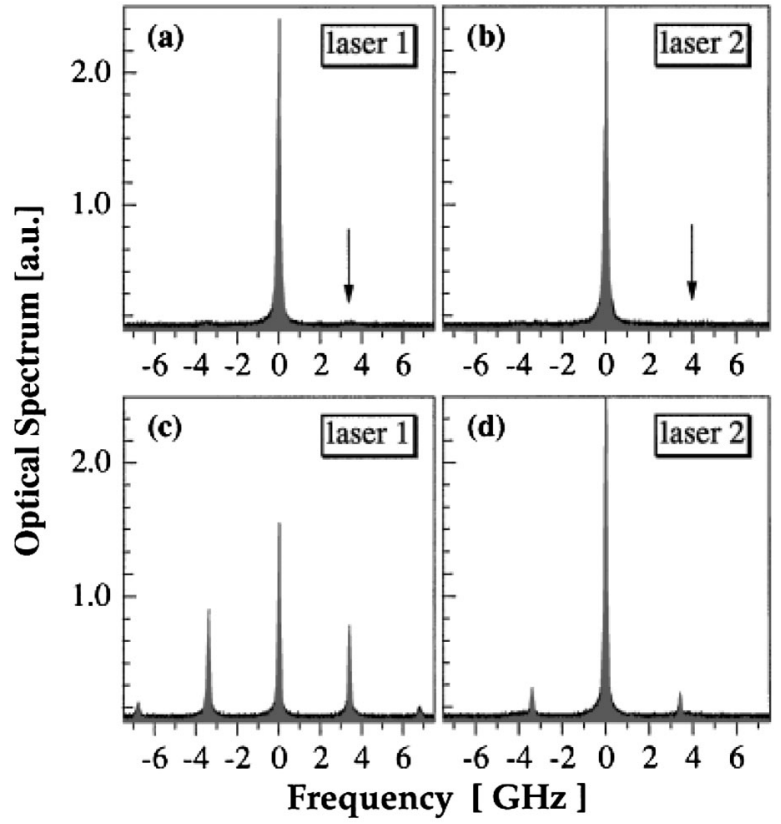

FIG. 14. Experimental optical spectra of the two lasers demonstrating localized synchronization. (a) and (b) depict the spectra without coupling, and (c) and (d) the ones with weak coupling. From Hohl et al., 1997.

average, for the same time. The synchronization that is established between the lasers is not complete but generalized, in the Abarbanel sense (Abarbanel, Rulkov, and Sushchik, 1996). This fact can be proven by unidirectionally coupling a third identical laser, used as a twin system, to one of the two lasers (this situation corresponds to $\kappa_{3,2}=0$ in Fig. 23). Results obtained numerically for two identical mutually coupled lasers and the attached test laser revealed identical synchronization between the test and one of the two lasers (the one that is not directly connected to it) (Van der Sande et al., 2008). These results were also found experimentally for the case of two mutually coupled Mackey-Glass electronic circuits (Soriano et al., 2012).

Figure 15 depicts, in comparison of experiment and modeling, how the maximum of the cross-correlation function increases as the coupling strength $\kappa_{c}$, normalized to the

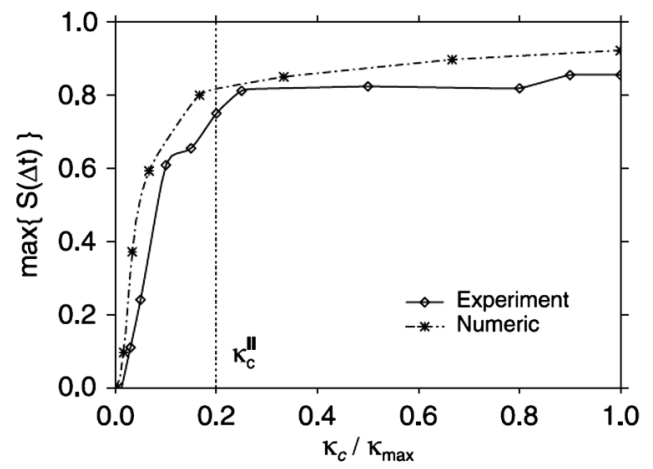

FIG. 15. Comparison of the maximum degree of correlation achieved as a function of the coupling strength for experiment and modeling. Both lasers are pumped at the solitary laser threshold. From Mulet et al., 2004. maximum attainable value in the experiment $\kappa_{\max }$, is increased. Below $\kappa_{c}^{\|}=\kappa_{c} / \kappa_{\max }=0.2$ the two lasers are already unstable, but do not clearly synchronize. Beyond this value, generalized synchronization is attained and maintained for larger values of the coupling strength.

When biasing both lasers at the same current close to the solitary laser threshold, the low-frequency intensity dropouts occur strongly correlated in both systems, however with a time lag of either $\tau_{c}$ or $-\tau_{c}$ between the two signals, as shown in Figs. 16 and 17, upper panel. The same characteristic can be seen at a much shorter time scale where subnanosecond pulsations occur (see Fig. 17, lower panel). In Fig. 17, lower panel, the delay $\tau_{c}$ has been compensated for. The cross-correlation function between the two output intensities plotted in Fig. 18 provides more insight into the underlying mechanism. It illustrates that the delay between the leading laser (leader) and the lagging laser (laggard) exactly corresponds to the coupling delay $\tau_{c}$, highlighting the occurrence of the spontaneous symmetry breaking in the system and yielding an achronal generalized synchronization. This fact is reflected in the height of the correlation peaks in Fig. 18 that are almost identical for $\pm \tau_{c}, \pm 2 \tau_{c}, \ldots$ but smaller than 1 . The small asymmetry present in the height of the peaks in Fig. 18(b) is due to small unavoidable mismatches between the two devices. The two main peaks in the cross-correlation function are identical (or almost identical) which is due to the change in the leader-laggard role in time. This fact is confirmed by computing the probability density function of the time shift between power dropouts of the two lasers. The time lag is defined by means of $\tau_{0}=\tau_{k}^{1}-\tau_{k}^{2}$, where $\tau_{j}^{k}$ stands for the $k_{\mathrm{th}}$ dropout time of laser $j$. To decide whether a power dropout occurs or not, a predefined threshold is assumed. Hence, positive (negative) $\tau_{0}$ means that laser 1 (2) drops before laser 2 (1). The probability distribution function of $\tau_{0}$, shown in Fig. 16, shows that most of the dropouts occur at times $\tau_{0} \sim \tau_{c}$, whereas larger times are unlikely.

The symmetry in the two peaks can be broken if a frequency detuning between the two lasers is induced (Heil, Fischer, Elsäßer, Mulet, and Mirasso, 2001), an asymmetric coupling strength is considered (Gonzalez, Torrent, and García-Ojalvo, 2007), or the lasers are biased differently (Deng et al., 2011). Under any of these conditions, a stable leader-laggard generalized synchronization is established.

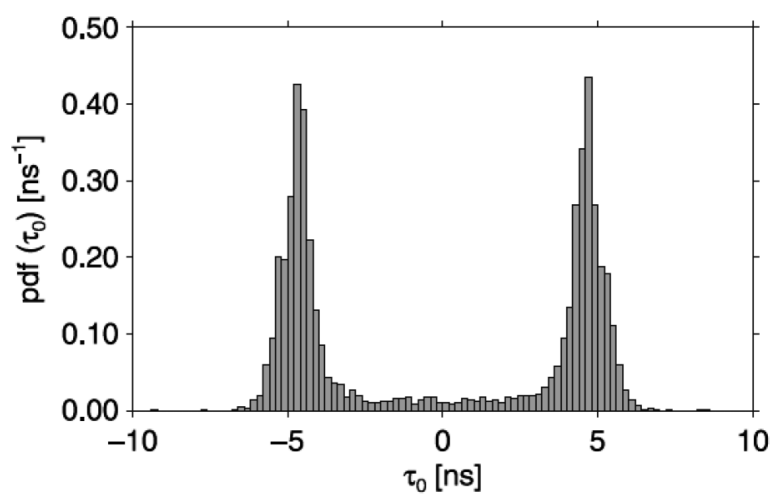

FIG. 16. Probability density function of the time shift between power dropouts of the two mutually coupled lasers. From Mulet et al., 2004. 

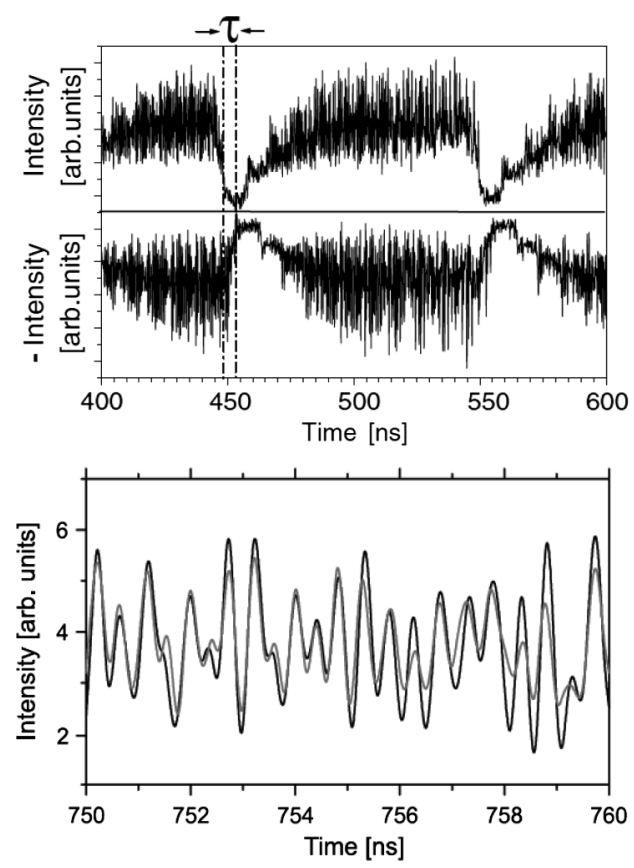

FIG. 17. Upper panel: Experimental intensity time series of the two lasers. The lower trace shows the inverted time series. Both lasers are pumped at $I=I_{\mathrm{th}}^{\text {sol }}$. Lower panel: Subnanosecond synchronized dynamics between two consecutive power dropouts with the delay-coupling time compensated for. From Heil, Fischer, Elsäßer, Mulet, and Mirasso, 2001.

Another aspect that can be observed from Fig. 18 is that the correlation coefficient corresponding to the isochronal identical synchronization is very small, in both the experiment and the simulations. The reason for its small value is that the solution, although existing, is unstable, as demonstrated both numerically (Mulet et al., 2004) and analytically (White, Matus, and Moloney, 2002; D'Huys et al., 2010; Englert et al., 2010; Flunkert et al., 2010). The correlation coefficient is not zero (or close to zero) at zero lag because the lasers were biased at threshold, operating in the LFF regime with the characteristic dropouts in the optical power (see Fig. 17). If the correlation coefficient is computed using only the dynamics between dropouts (Klein, Gross, Rosenbluh et al., 2006) or for higher bias currents, one obtains negligible values at zero lag.

Numerical simulations have mostly been performed based on a modified version of the Lang-Kobayashi equations, yielding qualitatively the same results as in the experiments as shown, for instance, in Fig. 18(a) (Heil, Fischer, Elsäßer, Mulet, and Mirasso, 2001; Mulet et al., 2004). A detailed derivation and analysis of this model, assuming weak to moderate coupling strength and single-mode operation for the two lasers, can be found in Mulet, Massoller, and Mirasso (2002), Erzgräber, Krauskopf, and Lenstra (2005), and Erzgräber et al. (2005). From the steady-state analysis, three different types of monochromatic solutions, usually called compound laser modes, were found: in-phase and antiphase symmetric solutions and asymmetric solutions. In the symmetric solutions, the two lasers oscillate with a relative phase that is restricted to being either 0 (in phase) or $\pi$ (antiphase). In spite of the high degree of symmetry in the system, asymmetric solutions, in which the gain in both lasers is
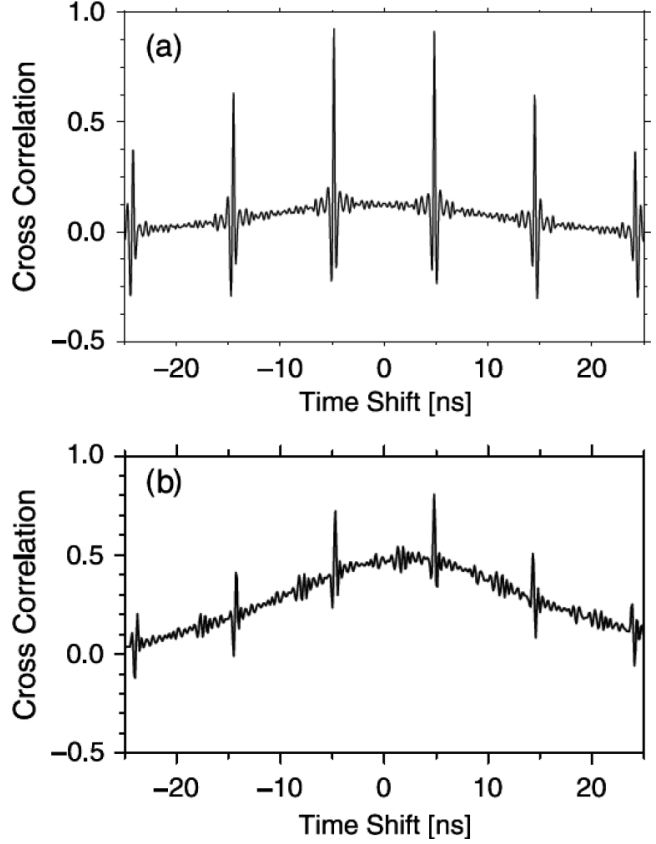

FIG. 18. (a) Numerical and (b) experimental cross-correlation functions. From Mulet et al., 2004.

different, were also found, although they turned out to be unstable (Mulet, Massoller, and Mirasso, 2002; Erzgräber, Krauskopf, and Lenstra, 2005; Erzgräber et al., 2005). It is worth mentioning that these compound laser modes form the underlying skeleton of the dynamical system; their stability and overall structure are found to be vital ingredients of the dynamics.

Analytical and numerical studies based on even simpler models were also carried out. In the limit of an infinitely long coupling delay, symmetric, antisymmetric, and asymmetric solutions were also found (Javaloyes, Mandel, and Pieroux, 2003). The stability of these solutions was determined partly analytically and partly numerically. In the opposite limit (Rogister and Blondel, 2004; Yanchuck, Schneider, and Recke, 2004), where the coupling delay is very small, and for the case of identical systems, synchronous, antisynchronous, and asynchronous cw solutions were observed (Yanchuck, Schneider, and Recke, 2004), the stability of which depends on the coupling strength and feedback phase.

Besides the many studies on bidirectionally coupled edgeemitting semiconductor lasers, other configurations, including semiconductor lasers with bidirectional optoelectronic coupling (Tang et al., 2004; Chiang, Chen, and Liu, 2005), mutually coupled vertical-cavity surface emitting lasers (Vicente et al., 2006; Li et al., 2007; Ozaki et al., 2009), fiber ring lasers (Rogers-Dakin et al., 2006), quantum dot lasers (Hegarty et al., 2007), and coupled DBR laser pairs (Vaughan et al., 2009) were investigated. When considering semiconductor lasers with bidirectional optoelectronic coupling, and depending on the operating conditions, the mutual coupling can quench their oscillations giving rise to the phenomenon of death by delay (Tang et al., 2004). This occurs when the two lasers self-oscillate when uncoupled and operate $\mathrm{cw}$ when being coupled, as seen in Fig. 19. In this figure the experimentally recorded time traces of the optical power of two mutually optoelectronically coupled 

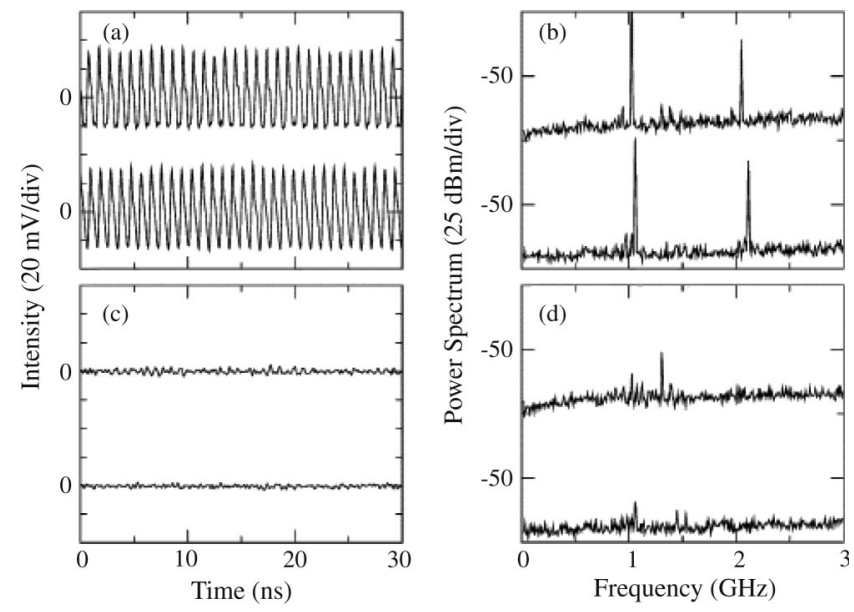

FIG. 19. Characteristics of "death by delay". (a), (b) Time series and power spectra, respectively, of the two lasers before the mutual coupling. (c), (d) Corresponding plots after the mutual coupling. From Tang et al., 2004.

self-oscillating lasers are plotted before and after the coupling is connected. Conversely, the mutual coupling can also induce complex dynamics. Torus and period-doubling bifurcations were found in such a system. From an exhaustive theoretical analysis, a quasiperiodic route to chaos with boundary crisis events was identified as the responsible mechanism leading the system from a regular to a complex behavior, even in the absence of self-feedback (Vicente et al., 2004). Although the chaotic waveforms are complex with broad spectra, generalized synchronization between the chaotic waveforms can be observed. Such synchronization is achieved due to the effect of the mutual coupling and the symmetric design between the two lasers. When asymmetric coupling strengths are implemented, a scaling law that relates the amplitudes of the oscillations and the coupling strengths was found, both numerically and experimentally (Kim et al., 2005). Interestingly, this study was inspired by the formal correspondence between a class of epidemic models and a class of the laser models, highlighting the interdisciplinary character of delay systems.

\section{Dynamics and synchronization of further bidirectionally coupled semiconductor laser configurations}

In most of the results described in Sec. III.C, the identical zero-lag synchronization solution exists, but is unstable when two dynamical systems are mutually coupled with a certain delay. This fact prevents the utilization of the system for bidirectional chaos-based optical communications (see Sec. V.D) and left the occurrence of zero-lag synchronization unexplained that was observed in the brain between widely separated cortical regions (Roelfsema et al., 1997) (see Sec. V.E). Different approaches were proposed to stabilize the zero-lag solution (see some of the proposed schemes in Figs. 22 and 23). In what follows we discuss the different configurations.

\section{Mutually coupled lasers subject to self-feedback}

The inclusion of a self-feedback loop in two mutually coupled lasers can stabilize the zero-lag solution under certain conditions. A feedback delay time that matches the

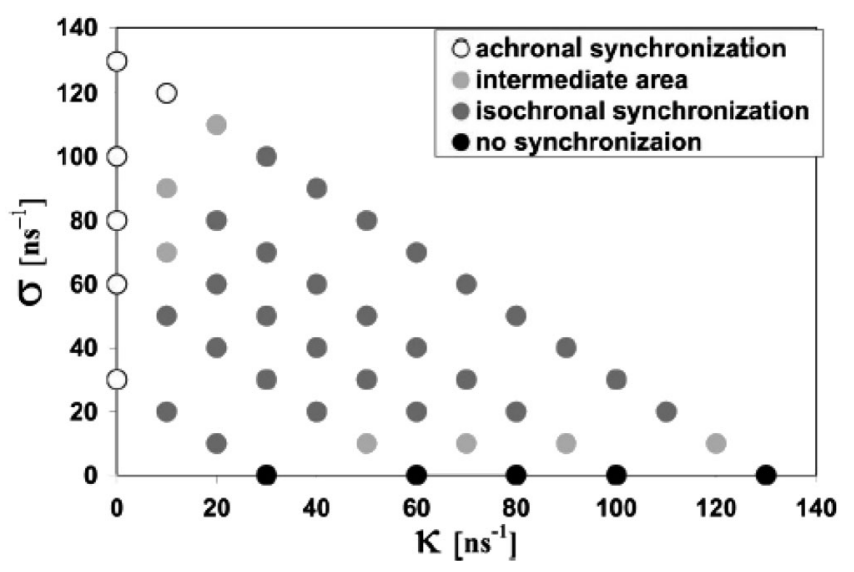

FIG. 20. Parameter space of coupling strength and self-feedback strength indicating the synchronization regions. From Klein, Gross, Rosenbluh et al., 2006.

coupling delay between the lasers was proven to stabilize the synchronized solution (Klein, Gross, Rosenbluh et al., 2006; Schwartz and Shaw, 2007). Under self-feedback, the two lasers play the same role in creating and maintaining synchronization, avoiding, at the same time, the symmetry breaking. Based on numerical simulations using a modified version of the LK equations, Klein, Gross, Rosenbluh et al. (2006) explored the region in the parameter space where isochronal synchronization occurs. In Fig. 20 a wide region in which isochronal synchronization occurs can be seen in the feedback strength versus coupling strength parameter space, although regions of achronal synchronization and no synchronization are also visible.

When the feedback delay time and the coupling time are different to each other, the zero-lag solution disappears except for a small mismatch between them (Martinez Avila and Rios Leite, 2009).

\section{Mutually coupled lasers subject to a common drive}

Another way to stabilize the zero-lag solution was proposed by Zhou and Roy (2007) using Ikeda ring oscillators, and Jiang et al. (2010) using semiconductor lasers. The two groups showed that two of these nonlinear elements can isochronously synchronize if they are symmetrically driven by a third nonlinear element. Figure 21 shows the synchronization diagram between the mutually coupled lasers as a function of the external driving strength induced by a third chaotic laser. A wide region of zero-lag synchronization is observed for values of driving strength larger than the mutual coupling strength (Jiang et al., 2010). This synchronization was found to be quite robust with respect to parameter mismatch between the two mutually coupled elements, the most critical being the frequency detuning between them. The scheme proposed by Jiang et al. (2010) is shown in Fig. 22(b).

Interestingly, this kind of setup also allows for zero-lag synchronization, if the external driving is a noise source (Gonzalez et al., 2011). If the external driving is suppressed, then the achronal solution emerges again, except the lasers are coupled with different delays. In this case, the zero-lag solution can be stabilized for certain integer ratios of the two delays (Englert et al., 2010; Zigzag et al., 2010). 


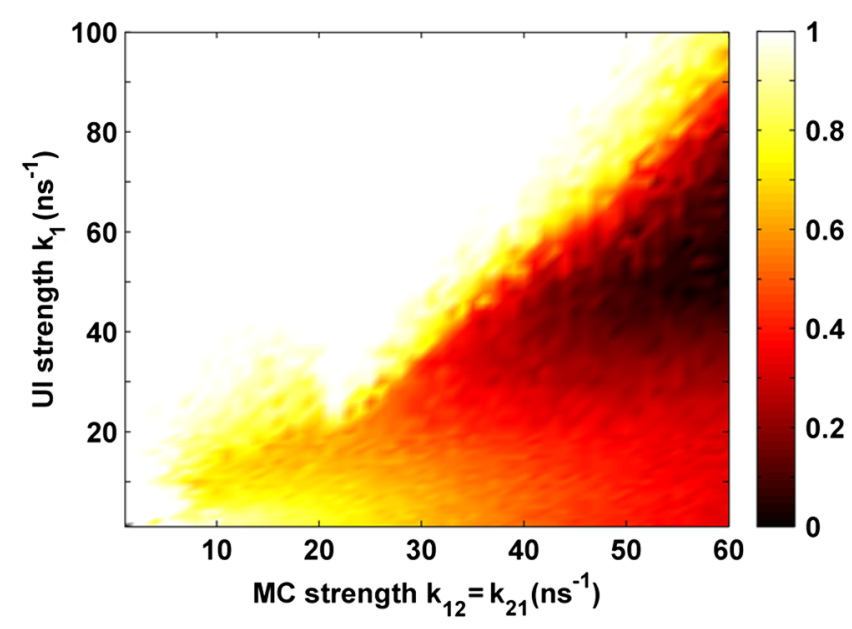

FIG. 21 (color online). Maximum of the cross-correlation coefficient between two mutually coupled lasers subject to an external common driving. From Jiang et al., 2010.

\section{Mutually coupled lasers through a relay element}

A third option is to introduce a relay element. One of the first experimental studies considered three laterally coupled lasers without delay (Terry et al., 1999), similar to the configuration described by Winful and Rahman (1990). It was shown, both experimentally and numerically, that identical synchronization between the outer lasers, but not with the central one, occurred. Unlike this case, we present in this section results related to the situation in which the dynamical elements, mainly semiconductor lasers, are physically separated, giving rise to significant coupling delays due to the finite propagation times of the signals. This configuration was first analyzed by Buric and Todorovic (2003) when studying the synchronization properties of three bidirectionally coupled hyperchaotic systems based on the Ikeda model. They found conditions for which the three elements identically synchronized as a function of the coupling strengths and the two delay times: the intrinsic delay of the Ikeda oscillators and the coupling delay.

The use of a mediator-based semiconductor laser was proposed, and experimentally studied, by Sivaprakasam et al. (2003) to compensate the delay time between a transmitter, a mediator, and a receiver. The transmitter was composed of a semiconductor laser subject to optical feedback from an external cavity, while the mediator and receiver were free-running lasers. The transmitter and mediator were mutually coupled with a certain delay and the output of the latter was unidirectionally coupled, with the same delay time, to the receiver. In this situation, generalized zero-lag synchronization was observed among the three elements.

In what follows, we consider a configuration of three mutually delay-coupled semiconductor lasers along a line, in such a way that the central element acts as a relay of the dynamics between the outer elements (see Fig. 23). Under the conditions that a third laser (Fischer et al., 2006) or a semitransparent mirror (Vicente, Mirasso, and Fischer, 2007) is placed between two face-to-face coupled semiconductor lasers, identical synchronization can be achieved. The stability of this solution depends, however, on certain laser parameters and operating conditions (see Sec. III.E).

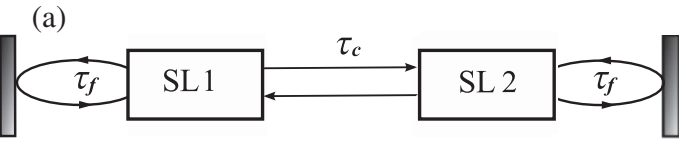

(b)

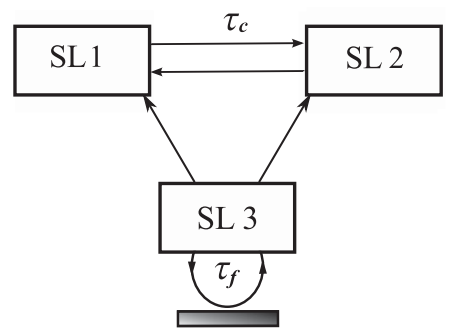

FIG. 22. Different schemes for stabilizing the isochronal solution. (a) Bidirectionally delay-coupled self-oscillating lasers. (b) Bidirectionally delay-coupled lasers driven by a third laser.

The experimental setup and the corresponding conditions for the numerical simulations comprise three identical semiconductor lasers (within parameter tolerances) connected via identical coupling delays $\tau_{i, j}=\tau_{c}$ (see Fig. 23). The coupling strengths $\kappa_{i, j}=\kappa_{c}$ were also assumed to be identical in all cases. The main results, obtained both experimentally and numerically, are summarized in Fig. 24. The most relevant feature is the occurrence of a collective behavior that gives rise to zero-lag identical synchronization between the outer lasers, mediated by the relay laser. This was surprising taking into account the spatial separation between the outer lasers. In modeling, this zero-lag identical solution was found for arbitrary long coupling delays, if the two branches had the same length. If a mismatch in delay times is introduced according to $\tau_{c, 1}=\tau_{1,2}=\tau_{2,1}$ and $\tau_{c, 2}=\tau_{2,3}=\tau_{3,2}$, keeping the other parameters symmetric, the identical synchronization remains stable; however, a time shift of $\tau_{c, 2}-\tau_{c, 1}$ between the lasers is observed. One might initially think that the synchronization between outer lasers is driven by the central element: the relay drives the outer lasers. This is not the case for the configuration shown in Fig. 23. The small asymmetry present in the cross-correlation functions between $+\tau_{c}$ and $-\tau_{c}$ [see Figs. 24(e) and 24(f)] is not due to the mismatch between the outer lasers (which is in any case unavoidable), but reveals that the central laser lags in the dynamics with respect to the outer ones. This was found by a careful inspection of the experimental times series and corroborated by numerical simulations of identical coupled lasers (Vicente, Mirasso, and Fischer, 2007). The lagging of the middle element is related to the fact that it receives more input than the outer ones. In the opposite situation, the middle element can lead the dynamics. In both cases, since the coupling induces the instability, as well as the

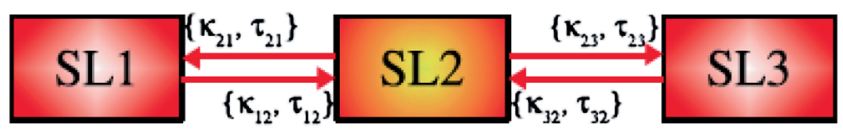

FIG. 23 (color online). Chain of three mutually coupled semiconductor lasers interacting bidirectionally with a time delay. 

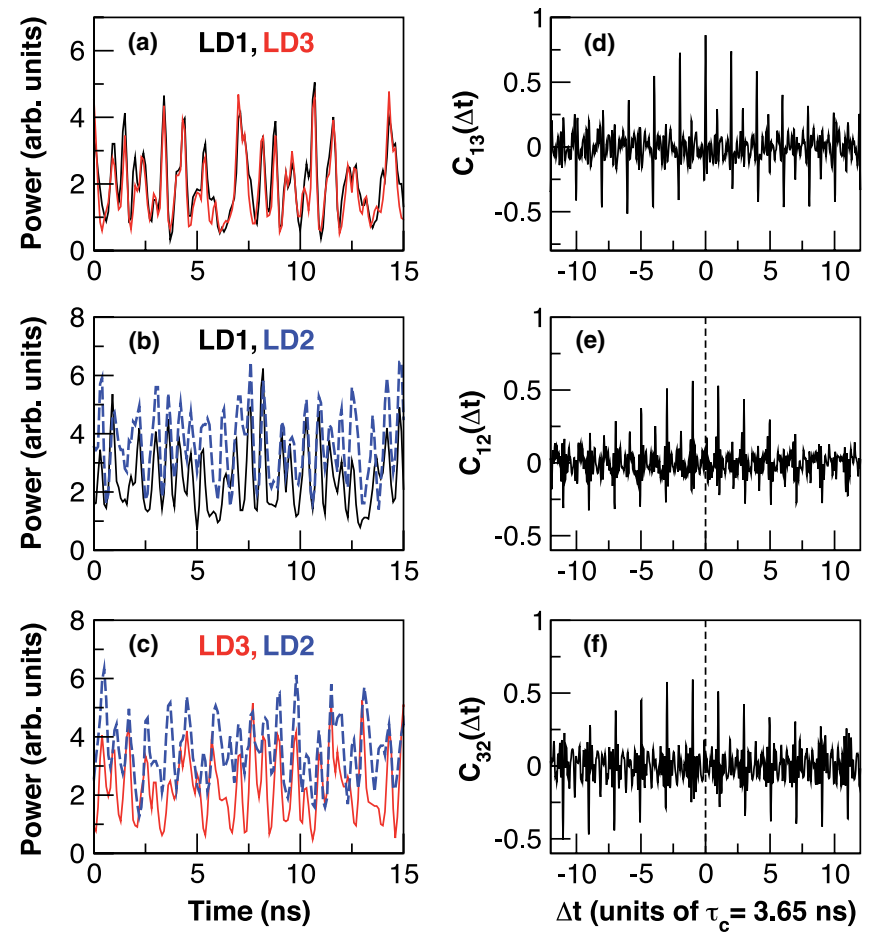

FIG. 24 (color online). (a)-(c) Time series (in pairs) of the output intensity of the lasers; (d)-(f) cross-correlation functions of the corresponding time series. The time series of the central laser have been shifted by the coupling delay to allow an easier comparison. Adapted from Fischer et al., 2006.

synchronization, a truly collective behavior, that cannot be explained by analyzing the individual elements, is established. This collective behavior emerges under involvement of the relay element that plays a crucial role; it feeds part of the light received from the outer lasers symmetrically to both outer lasers.

Besides the configuration described previously, it is also possible to achieve identical synchronization between two spatially separated semiconductor lasers by relaying their dynamics via a passive element such as a semitransparent mirror (Vicente, Mirasso, and Fischer, 2007). Using numerical simulation based on a modified version of the Lang-Kobayashi equations, it was reported that identical synchronization can be obtained, even if the two branches connecting the outer lasers have different lengths. In the latter case, a time shift according to the difference between the coupling delays in the two branches was found. Although the synchronization remains stable, the delay mismatch significantly affects the emerging dynamics (Hicke et al., 2011). In turn, a coupling mismatch deteriorates the stability of the synchronized solution, but does not affect the synchronized dynamics much.

The identically synchronized solution is very sensitive to the optical phases of the light in the cavities and consequently they have to be carefully adjusted (Ruiz-Oliveras et al., 2011; Flunkert and Schöll, 2012). This limitation can be overcome by using scalar feedback and coupling that can be generated, e.g., using electro-optical devices. Recent experiments considered mutually delay-coupled electro-optic oscillators for which robust identical synchronization was obtained for both symmetric and strongly asymmetric timing of the mutual coupling (Peil, Larger, and Fischer, 2007). In Sec. III.E, we discuss the stability of the identical synchronization solution.

\section{E. Stability analysis of the identical (zero-lag) solution}

Extensive numerical simulations were carried out to study the robustness of the identical synchronized solutions of three mutually delay-coupled lasers in terms of different laser parameters and operating conditions (Vicente et al., 2008). In general, it is found that while the matching between outer elements (in terms of injection currents, emission frequency, and laser parameters) is very important, the synchronization is very robust against mismatch with respect to the central element. The stability of the identical synchronized solution was proven analytically in terms of the local dynamics of each laser (Landsman and Schwartz, 2007). Ideas from generalized synchronization were used to explain the complete synchronization in the presence of long coupling delays. The results reported by Landsman and Schwartz (2007) explain and predict the dependence of the synchronized solution on various parameters, such as coupling delays, coupling strengths, etc.

To gain insight into the properties of the synchronization of small networks or network motifs, delay-coupled StuartLandau oscillators, as generic limit-cycle oscillators, were considered (D'Huys et al., 2010). The role played by both amplitude and phase instabilities in producing symmetrybreaking or symmetry-restoring transitions were analyzed using analytical and numerical methods. When two oscillators are mutually coupled with delay, in-phase and antiphase modes are possible under weak coupling. When the amplitude oscillations occur, however, only one type of oscillation is possible. When adding feedback to the two oscillators, either by adding extra feedback loops or by placing a relay element between them, the zero-lag solution can be stabilized, if the feedback and coupling delays coincide.

Recent theoretical studies (Flunkert et al., 2009) showed, however, that the synchronized solution can suffer from short intervals of desynchronization in the presence of relay elements, either passive or active. Depending on the coupling parameters the system exhibits bubbling, i.e., noise-induced desynchronization, or on-off intermittency. These episodes of desynchronization dynamics, that can occur in both CC and LFF regimes, were found to be related to the transverse instability of some of the compound cavity antimodes (Flunkert et al., 2009). The instability related to on-off intermittency is illustrated via the transverse Lyapunov exponent, as depicted in Fig. 25. Bubbling even occurs in regimes in which the maximum transverse Lyapunov exponent is negative.

Recent experimental results, using two semiconductor lasers mutually coupled via a passive relay loop, confirmed these findings. While the system exhibited identical chaos synchronization, it was found that the synchronized solution could suffer short periods of synchronization losses (see Fig. 26) associated with bubbling events, the frequency of which increases with increasing pump current (Tiana-Alsina et al., 2012).

The stability of the synchronized solution in delay-coupled networks of identical units was also investigated in terms of 


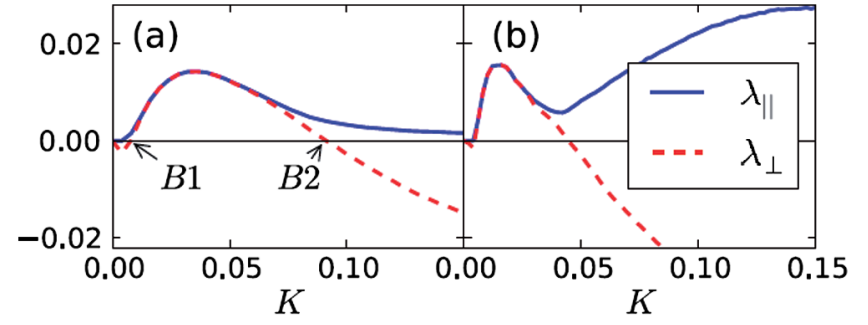

FIG. 25 (color online). Maximum transversal (dashed) and parallel (solid) Lyapunov exponents as a function of the feedback strength $\kappa$ for (a) a passive relay and (b) an active relay. At the two blowout bifurcations $B 1$ and $B 2$ the maximum transversal Lyapunov exponent of the chaotic attractor changes sign. From Flunkert et al., 2009.

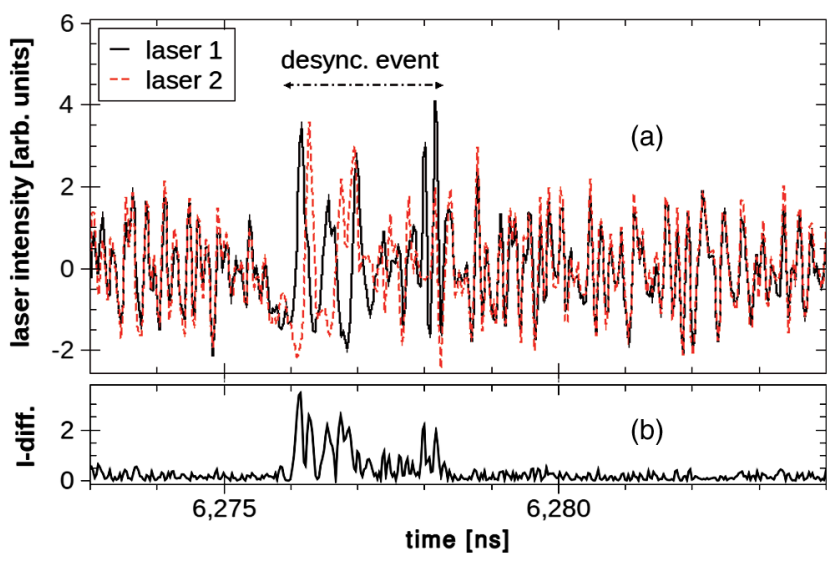

FIG. 26 (color online). (a) Experimental time series of synchronized fast intensity dynamics in the coherence collapse regime. A short desynchronization event is highlighted. The pump current corresponds to 1.25 , the threshold current of the solitary laser. (b) Corresponding normalized intensity difference (synchronization error). From Tiana-Alsina et al., 2012.

the master stability function (Flunkert et al., 2010) that will be discussed in more detail in Sec. IV.D. Using this method, the synchronizability of the network can, for large coupling delays, be directly related to the spectral properties of the network topology itself. For two bidirectionally coupled systems it can be shown that zero-lag identical chaos synchronization is not possible, when the coupling delay is large (larger than the characteristic time scale of the system), while for the relay topologies chaos synchronization is possible.

\section{F. Ordering role of noise}

We have seen in Sec. II.G.2 that noise can have an ordering and potentially even constructive role in the dynamics of nonlinear systems, in particular, in semiconductor lasers with feedback. Another potentially constructive effect of noise is to induce the synchronization of coupled systems (Maritan and Banavar, 1994; Sánchez, Matías, and PérezMuñuzuri, 1997; Toral et al., 2001; Zhou and Kurths, 2002) by which a certain amount of common noise leads coupled systems to collapse onto the same trajectory. This property can be used to achieve the zero-lag synchronization solution in a system of two uncoupled lasers with a common external optical white Gaussian-noise signal (Wieczorek, 2009) or two bidirectionally coupled semiconductor lasers, when the pump currents of the two lasers are subject to a common source (Gonzalez et al., 2011). In the latter and for small noise levels, the lasers exhibit the standard leader-laggard synchronization regime, as described in Sec. III.C. For large enough noise intensity, however, the lasers reach a common output without lag between them, stabilizing the identical (zero-lag) solution.

This zero-lag synchronized state is, nevertheless, different from the intrinsic dynamics of the lasers. In particular, the cross correlation of the signals showed a noticeable broadening of its maximum peak at zero lag, in comparison with the peaks (at nonzero lag) that exist in the absence of noise. The origin of this broadening can be established by analyzing numerical simulations of the system. This allows for an arbitrarily large temporal resolution of the dynamics and an infinite bandwidth of the noise being added to the lasers' pump currents. The simulations showed that it is the nonzero correlation time of the noise that causes the differences between the dynamics of the noise-driven and noise-free coupled lasers. For large noise correlation time, the systemreacts only to the fluctuations in its slow dynamics, whereas, in the limit of very small noise correlation time, both the slow and fast dynamics can respond (Gonzalez et al., 2011).

A second example of the ordering influence of noise in coupled laser systems can be found when studying the response of two mutually injected lasers to an external signal. Early experiments showed that coupling substantially enhances the response of the lasers to an external modulation (Buldu et al., 2002), in comparison with the response of a single laser with feedback (Sukow and Gauthier, 2000). A similar effect can be observed experimentally when the input signal affecting the lasers is complex, i.e., it consists of two harmonics of a missing fundamental. As in the case of a single laser with feedback, discussed in Sec. II.G.2, coupling mediates the response of the laser to the missing fundamental. This represents another instance of ghost stochastic resonance (Buldú et al., 2005). This happens even when the two input frequencies are applied separately to the two lasers (González, Buldú et al., 2007). Again, noise is necessary for the phenomenon to occur and is provided by the internal dynamics of the lasers. This observation constitutes a basic example of how coupled systems can process complex distributed information.

\section{NETWORK MOTIFS AND SMALL NETWORKS OF DELAY-COUPLED SEMICONDUCTOR LASERS}

So far we have examined the dynamical properties, in particular, the synchronization characteristics, of two coupled lasers connected either directly or via a third relay element. As seen, this situation leads to a rich variety of complex phenomena, including coupling-induced instabilities, symmetry breaking, zero-lag synchronization, and bubbling. From a different angle, recent advances in complexity science have highlighted the importance of networks, containing a relatively large number of coupled elements, as sources of complex phenomena, first from the point of view of their topology (Strogatz, 2001) and later from a dynamical 
perspective (Boccaletti et al., 2006). Networks are ubiquitous in both natural systems (gene regulation, cell signaling, neuronal tissue, ecosystems, social interactions) and technology (electric grid, Internet), and their behavior can be addressed with the methods of statistical physics (Albert and Barabási, 2002). A complementary, reductionist approach is based on decomposing the network into modules consisting of a small number of coupled elements in order to reach an understanding of the system's behavior from the bottom up. In this section, we overview the efforts made so far toward the comprehension of the dynamics of laser networks, including not only module-based but also global approaches. These studies reveal important correlates between coupling topology and dynamical behavior and bring to light synchronization mechanisms that are qualitatively different from the case of two coupled lasers.

\section{A. Laser motifs}

In many situations, complex networks can be decomposed into communities of nodes which are more closely related to each other, either structurally or functionally, than to the rest of the nodes in the network (Girvan and Newman, 2002). Given their relative isolation, one can expect that studying the dynamics of those modules should help in reaching an understanding of the behavior of the complete network. Figure 27 shows, as an example, the complete set of potential modules composed of three nodes, distinguishing between unidirectionally and bidirectional links. Six out of the 13 different modules correspond to linear chains, while the other seven are ring structures. In Sec. III.D we analyzed the behavior of linear structures, mainly the bidirectional chain of three coupled lasers corresponding to module 4, and observed that in the presence of sufficient structural symmetry this module leads in a natural way to zero-lag synchronization (Fischer et al., 2006). We also showed that this dynamical
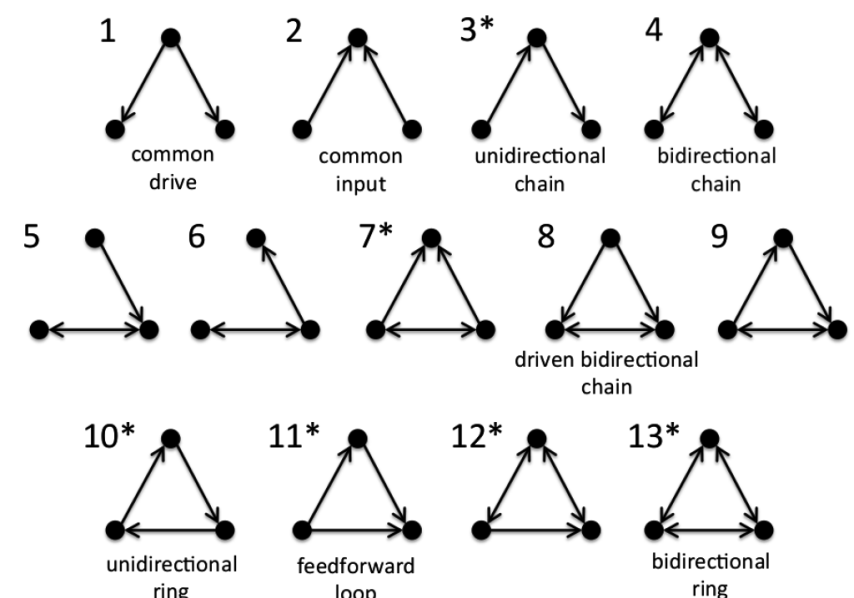

FIG. 27. Scheme of all possible modules containing three nodes. Standard modules and those most frequently discussed in this review are named. An asterisk denotes that the module is a motif, namely, that it occurs more frequently than randomly in selected real-life networks, as calculated by Milo et al. (2002): module 3 is a motif in food webs, 7, 12, and 13 in the Internet, 10 in electronic circuits, and 11 in transcriptional, neuronal, and electronic networks. regime can be stabilized as well by an architecture such as the one of module 8 , in which two bidirectionally coupled oscillators are driven by a common element (Zhou and Roy, 2007).

The relevance of the modularity hypothesis is reflected in the fact that some modules are found in real networks much more frequently than given by chance. These overrepresented modules were termed network motifs by Milo et al. (2002) and are marked with an asterisk in Fig. 27. Note that five out of the seven circular modules are motifs in at least one particular type of natural network (see figure caption for details). In particular, the unidirectional and bidirectional ring structures (modules 10 and 13, respectively) have been identified as motifs. In fact, the dynamics of these structures in the case of semiconductor lasers being the nodes has been studied in detail, both theoretically and experimentally, as discussed in Sec. IV.B.

\section{B. Synchronization of semiconductor laser rings}

In previous sections it was shown that feedback and bidirectional coupling lead, each on their own, to instabilities in the dynamics of semiconductor lasers. Numerical simulations of a modified LK model have shown that three lasers coupled unidirectionally in a ring structure (module 10 in Fig. 27) exhibit a similar coupling-induced instability (Buldú, Torrent, and Garcia-Ojalvo, 2007). In particular, this coupling configuration leads to synchronized low-frequency fluctuations with leader-laggard dynamics. This dynamics is characterized by power dropouts occurring in all three lasers almost simultaneously. The dropouts are separated by time intervals equal to the coupling time between the corresponding laser pair. Which laser exhibits the first dropout changes randomly. When the coupling between neighboring lasers is bidirectional, zero-lag synchronization arises, provided the parameter mismatch is not too large (Buldú, Torrent, and Garcia-Ojalvo, 2007).

In order to understand this behavior, D'Huys et al. (2011) performed a linear stability analysis on a set of Stuart-Landau oscillators coupled in a ring, both unidirectionally and bidirectionally. This system exhibits a Hopf bifurcation (Pikovsky, Rosemblum, and Kurths, 2001; Guo and Huang, 2007) as the coupling strength increases, even in the case of instantaneous coupling. This bifurcation results from an instability transverse to the synchronization manifold and thus leads to outof-phase behavior. The delay causes an additional instability parallel to the synchronization manifold, which leads to inphase oscillations. Interestingly, while in the unidirectional case the parallel instability occurs after the transverse one, and thus identical synchronization is not observed (D'Huys et al., 2010); the opposite may happen in the bidirectional case for sufficiently long time delay, therefore leading to inphase oscillations (which can be interpreted as zero-lag synchronization). Consequently, coupling delays in a bidirectional ring of three oscillators enhance symmetric behavior in the dynamics, something that was also reported in simpler network models of Kuramoto oscillators (D'Huys et al., 2008).

The analytical study of D'Huys et al. (2011) qualitatively agrees with the numerical observations of Buldú, Torrent, and Garcia-Ojalvo (2007). It can therefore be expected that the 
(a)
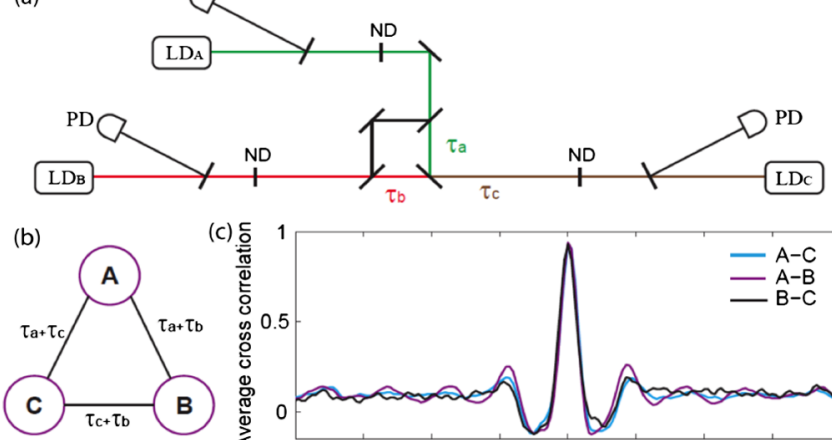

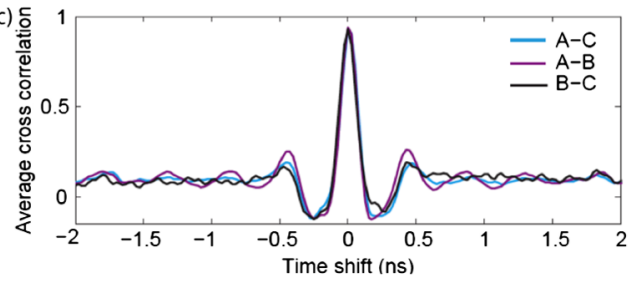

FIG. 28 (color online). Experimental observation of zero-lag synchronization in a bidirectional ring of three lasers. (a) Experimental setup, (b) scheme of the resulting laser motif, and (c) average crosscorrelation function of the three laser pairs. From Aviad et al., 2012.

behavior reported above (in particular, the zero-lag identical synchronization in bidirectional rings) should be observed experimentally in rings of coupled semiconductor lasers. Recent experiments by Aviad et al. (2012) confirm this expectation. A diagram of the experimental setup is shown in Fig. 28(a). Three Fabry-Perot semiconductor lasers, operating in the visible range, are coupled optically via a set of beam splitters arranged to allow for mutual injection between all pairs of lasers. The scheme of the resulting laser motif is shown in Fig. 28(b). For the experimental conditions chosen, the lasers operate in the LFF regime. When the three coupling delays are equal, $\tau_{a}=\tau_{b}=\tau_{c}$, zero-lag synchronization is observed among the three lasers, as shown in Fig. 28(c). The plot shows the cross-correlation functions of the three laser pairs, which exhibit a clear maximum close to unity at zero time lag. The correlation was computed using a time series that did not contain power dropouts, since the synchronization quality decreases considerably during the dropout events.

\section{Sublattice synchronization}

So far we have seen that modules composed of three bidirectionally coupled lasers, in both a linear chain and a ring, readily exhibit identical synchronization. When the number of lasers in the module is increased to four, the even character of the network allows for a new dynamical regime, in which the network can be decomposed into two identical sublattices (containing lasers that are not directly coupled), within which the lasers are identically synchronized at zero lag, while between elements in different sublattices generalized synchronization might occur. Furthermore, there is no well-defined leader or laggard sublattice; the cross correlation between them is symmetric. This behavior, termed sublattice synchronization, is a consequence of the symmetry properties of the network and was observed theoretically in coupled chaotic maps (Kestler, Kinzel, and Kanter, 2007), and experimentally in coupled semiconductor (Aviad et al., 2012) and solid-state (Nixon et al., 2011) lasers. The experimental observation in semiconductor lasers is shown in Fig. 29. As in the case of Fig. 28, four Fabry-Perot lasers operating in the visible wavelength regime are coupled via mutual injection of their respective optical fields [Fig. 29(a)], in such a way that they form a ring in which each laser is coupled to its two immediate neighbors [Fig. 29(b)]. Here the position of the beam splitters is such that the delays $\tau_{A}$ and $\tau_{B}$, directly associated to lasers A and B (which are not coupled directly), respectively, are almost identical, while the other two delays $\tau_{C}$ and $\tau_{D}$ are different from each other and from $\tau_{A} \simeq \tau_{B}$. The resulting dynamical regime corresponds to sublattice synchronization, in the sense that lasers $\mathrm{A}$ and $\mathrm{B}$, and lasers $\mathrm{C}$ and $\mathrm{D}$, which are not directly coupled to each other, are synchronized with each other. The former are synchronized at approximately zero lag since $\tau_{A} \simeq$ $\tau_{B}$. This can be seen in Fig. 29(c) in terms of the average cross correlation between the two laser pairs. Note that in both cases the maximum cross correlation is very close to 1 . Again, and as in the case of Fig. 28, the LFF regime was avoided to not get desynchronization events.

The concept of sublattice synchronization can be extended to larger number elements in the network than described so far and does not require a ring geometry. First experiments, not on chaos synchronization but on phase locking, were performed using Nd:YAG as gain medium, according to the setup shown in the top panel of Fig. 30. The lower panels of Fig. 30 depict experimental observations of Nixon et al. (2011) in a system of coupled solid-state lasers. A mask with $N$ apertures, placed close to one of the two end mirrors of the laser cavity, limits emission to $N$ independent laser beams within the crystal. These beams are then coupled via an external cavity through four coupling mirrors $R_{1-4}$, whose relative angular orientations allow the design of multiple coupling configurations. The phase locking between the lasers can be measured by means of the far-field intensity emitted by the array: in the absence of phase locking the
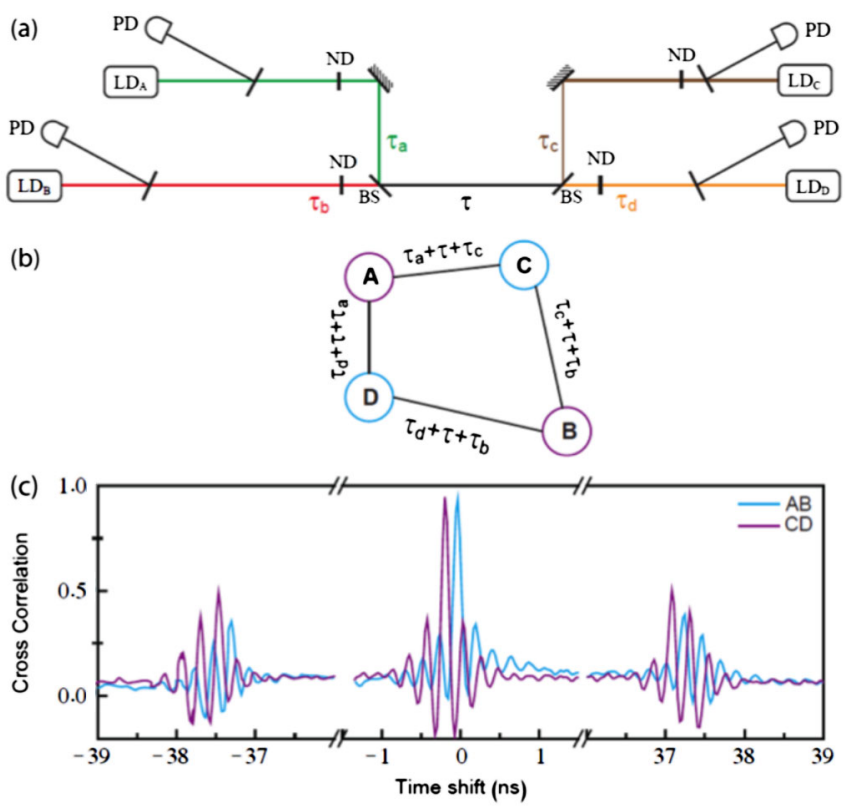

FIG. 29 (color online). Experimental observation of sublattice synchronization in a bidirectional ring of four lasers. (a) Experimental setup, (b) scheme of the resulting laser motif, and (c) average cross-correlation function of the two laser pairs. From Aviad et al., 2012. 

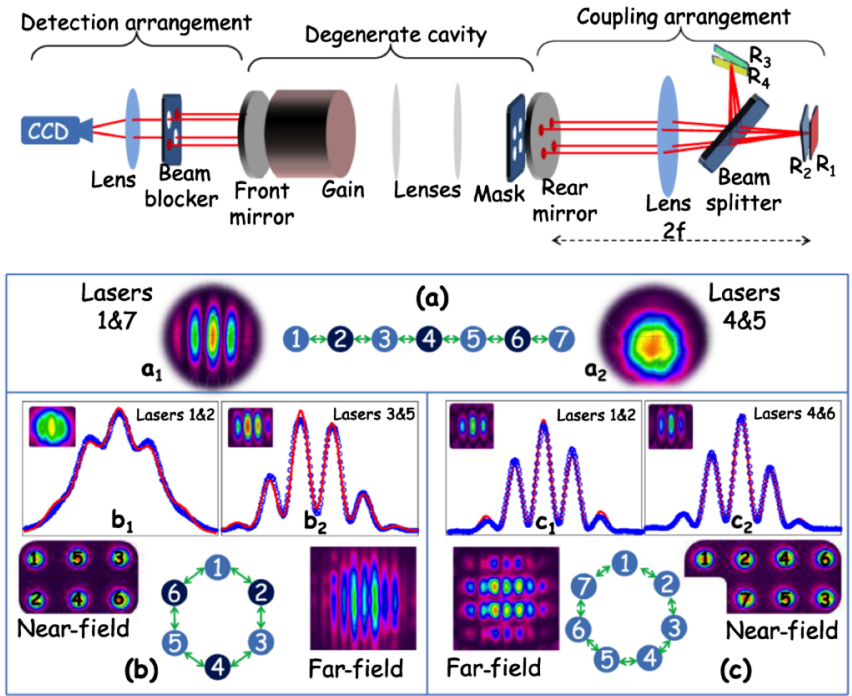

FIG. 30 (color online). Experimental observation of sublattice phase locking in different networks of delay-coupled solid-state lasers. Top panel: experimental setup. Bottom panel: (a) Linear chain of seven lasers (center), showing the far-field intensity profiles of lasers 1 and 7 (left) and 4 and 5 (right); (b) ring of six lasers; and (c) ring of seven lasers. Panels b1, b2, c1, and c2 show the cross section of the far-field intensity profiles of laser pairs (given in the plots). In all the network schemes, lasers with the same level of blue are phase locked with each other. From Nixon et al., 2011.

far-field emission is simply a sum of the intensity of all lasers in the array; in the presence of phase locking interference appears and a fringe pattern is observed (Fabiny et al., 1993; Ronen et al., 2008).

This experimental setup allows for a highly flexible design of the coupling configuration. Figure 30(a) shows the behavior of a chain of seven bidirectionally delay-coupled lasers. Similar to the case of the three lasers discussed above, the outer lasers 1 and 7 are phase locked as revealed by the fringe interference pattern shown at the left of the plot. In contrast, the two neighboring lasers such as 4 and 5 do not show phase locking between each other, with the far-field pattern being unstructured (plot at the right of the panel). This has been considered as another example of sublattice synchronization (Nixon et al., 2011), this time in a linear bidirectional chain, resulting from the symmetry of the coupling topology. This type of dynamics was previously conjectured, on the basis of simulations of the LK model, by Yanchuk, Stefanski et al. (2006). In the case of a ring, the symmetry considerations are different depending on the number of lasers. When the ring is formed by six lasers (bottom left panel in Fig. 30), the network can be decomposed into two effectively identical sublattices (formed by lasers 1-3-5 and 2-4-6, respectively) which identically synchronize within a sublattice, but not across sublattices, as revealed by the cross sections of the far-field intensity patterns of non-neighboring lasers 3 and 5 (panel b2), and neighboring lasers 1 and 2 (panel b1), respectively. Thus, sublattice synchronization in a ring arises for an even number of lasers. This behavior has also been reported in generic models of delay-coupled limit-cycle oscillators, in the form of rotating waves that persist for a large number of nodes in the ring (Dodla, Sen, and Johnston, 2004; Perlikowski et al., 2010). These rotating waves have also been observed experimentally in rings of coupled biological oscillators by Takamatsu et al. (2001). Returning to the experimental situation of Fig. 30, when the number of lasers in the ring is odd (bottom right panel of the figure), phase locking arises between all lasers. This is illustrated by the fringe patterns, appearing in the far field for all laser pairs (examples are shown for non-neighboring and neighboring lasers in plots $\mathrm{c} 1$ and $\mathrm{c} 2$ of that panel).

So far we have not considered the existence of selffeedback in the laser modules discussed in this section. The bifurcation behavior of rings of bidirectionally delay-coupled oscillators with feedback has been studied extensively from an analytical perspective by Yuan and Campbell (2004) and Bungay and Campbell (2007). Sublattice synchronization, on the basis of simulations of networks of chaotic maps, was found to exist in rings of four oscillators subject to feedback (Kestler et al., 2008). An experimental study by González, Masoller et al. (2007) revealed a route to synchronization via clustering in an array of three semiconductor lasers with feedback and all-to-all coupling, with nonhomogeneous coupling, feedback strengths, and delay times.

\section{Toward large laser networks}

The dynamical behaviors observed in the small laser modules can, in some cases, serve as building blocks that help in the understanding of the phenomena existing in larger networks. Sublattice synchronization, for instance, has been reported in triangular networks of locally and bidirectionally coupled chaotic maps, which can be decomposed into three identical sublattices (Kestler et al., 2008). Even when the connectivity topology throughout the network is heterogeneous (in terms of the node of the links and the coupling directionality), Kestler et al. (2008) reported that such clustering dynamics can be propagated through arbitrarily large lattices, in the form of what they called chaotic spreading motifs.

Applying the concept of sublattice synchronization, arbitrarily large networks can be decomposed into subsets of elements that receive the same input. This allows an approach toward synchronization of coupled networks of generic chaotic elements with multiple loops, in what constitutes an example of a nonlocal mechanism of synchronization (Kanter, Kopelowitz et al., 2011). The number of clusters is determined by the greatest common divisor (GCD) of the lengths of different directed loops in the corresponding graphs and can be much larger than 2 (Kanter, Zigzag et al., 2011). If the GCD is 1, identical synchronization of the whole network is possible. The minimum number of nodes that can illustrate this concept is 3 for heterogeneous delays and 4 in the case of homogeneous delays (Kanter, Zigzag et al., 2011). The GCD concept was first introduced for neuronal networks, as described in detail in Sec. V.E, and up to now has mostly been studied numerically. It is important to note that the GCD concept introduces a necessary, not a sufficient, condition for synchronization. Subsequently, analytical studies of networks of Bernoulli maps have been performed, restricted to non-negative coupling matrices with 
constant row sum and dynamics in the weak chaos regime (Englert et al., 2011). In this case a more rigorous condition for identical chaos synchronization has been derived which was later proven to hold also for continuous dynamical systems in the limit of long delays (Heiligenthal et al., 2011). It relates the synchronizability of the network to the gap between the first two eigenvalues of the coupling matrix. Comparing this condition with numerical simulations of networks of semiconductor lasers, following a LK-equationsbased approach, a reasonably good agreement was found (Englert et al., 2011). While experimental demonstrations of the GCD concept for chaos synchronization of semiconductor lasers are lacking, recent experimental work (Nixon et al., 2012) verified it for phase locking of up to 16 solidstate lasers using the configuration as depicted in Fig. 30.

Arrays of semiconductor lasers globally coupled with delay via an external mirror were seen to exhibit chaos synchronization for large enough feedback strength (GarciaOjalvo et al., 1999). A theoretical analysis of this system showed that the time delay induces in-phase synchronization of all the lasers for different types of local dynamics, including steady, periodic, and chaotic dynamics (Kozyreff, Vladimirov, and Mandel, 2000). In the case of an odd number of lasers, local extinction of the dynamics of one of the lasers is observed (Kozyreff, Vladimirov, and Mandel, 2001).

A recent approach toward the synchronization of a large number of semiconductor lasers involved the use of a starcoupling configuration, in which $M-1$ lasers are coupled bidirectionally to a central (hub) laser. Numerical simulations of this network, using a generalized LK model, revealed that the lasers undergo destabilization of the steady dynamics due to the coupling, and that this dynamics can be synchronized among all the outer lasers provided the number of coupled lasers is sufficiently large (Zamora-Munt et al., 2010), and the power of the hub can be scaled. This type of crowd synchrony was previously reported in civil engineering (Strogatz et al., 2005) and nonlinear chemistry (Taylor, et al., 2009). The plots in Fig. 31 show the coherent intensity $\langle I\rangle$ of the laser array, normalized to the number of lasers $M$, as $M$ increases. The coherent intensity is defined as the intensity of the total electric field of all lasers and is equal to zero in the unsynchronized case. The figure shows a transition from unsynchronized to identically synchronized behavior as the number of lasers in the network increases, for different values
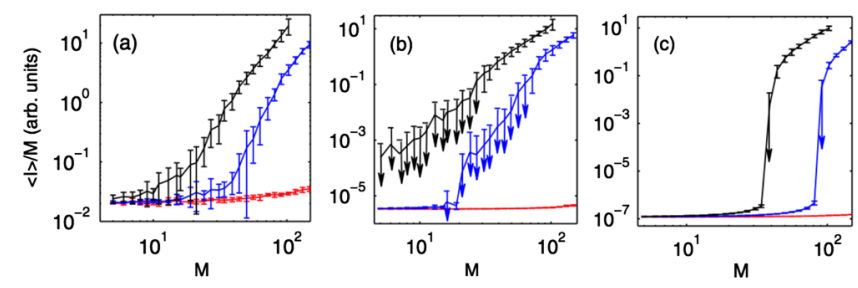

FIG. 31 (color online). Crowd synchronization in a star-coupled laser network. The normalized pump current of the hub laser is fixed to 0.4 in all three plots ( 1 corresponding to the threshold current in the solitary case), while that of the outer lasers decreases from left to right: (a) 1.02, (b) 0.99 , and (c) 0.7. Three values of the coupling strength (assumed equal for all links) are considered: $10 \mathrm{~ns}^{-1}$ is represented in light gray, $20 \mathrm{~ns}^{-1}$ in dark gray, and $30 \mathrm{~ns}^{-1}$ in black. From Zamora-Munt et al., 2010. of the pump current of the outer lasers, and for various coupling strengths. Two types of transitions occur depending on the value of the pump current of the outer lasers: when these lasers are pumped above their solitary threshold [Fig. 31(a)], the synchronization transition is gradual. In contrast, when the outer lasers are pumped below their solitary threshold, the transition is sharp. Similar zero-lag synchronization between the outer elements in a star configuration was reported in coupled fiber lasers, on the basis of numerical simulations, by Lindley and Schwartz (2011). A recent extension to multilayer laser networks was also introduced (Cohen, Rosenbluh, and Kanter, 2012).

Synchronization can also organize networks so that different dynamical behaviors coexist. This is the case of group synchronization, in which the network nodes are distributed in groups, with synchronous dynamics emerging within each group, while the different groups exhibit different dynamics (Sorrentino and Ott, 2007; Dahms, Lehnert, and Schöll, 2012). In that sense, group synchronization is more general than cluster or sublattice synchronization and can emerge under very general network topologies.

A different concept for the realization of complex laser networks was proposed by Amann et al. (2008), based on two-mode Fabry-Perot lasers (Osborne et al., 2007). By spatially varying the refractive index of a Fabry-Perot laser diode, Osborne et al. (2007) could tailor the spectrum of the laser diode to realize two-color lasers with predetermined lasing modes. Using a simple physical all-to-all coupling of many of such lasers with precisely tuned mode spacing, it would then in principle be possible to realize arbitrarily complex coupling topologies. In this case, the network nodes would correspond to lasing frequencies. A laser that operates on two frequencies then corresponds to a link between these nodes. The realizability and possible applications of such networks are under investigation.

\section{E. Stability of laser network synchronization}

So far we have seen that synchronized states can arise in laser networks even in the presence of, or even thanks to, the existence of delay in the coupling. In order to establish the limiting values of the delay for which the identically synchronized state exists, it is necessary to perform a stability analysis of that state. A technique that has been commonly used in that respect is the so-called master stability function. The method was initially introduced by Pecora and Carroll (1998) in networks of identical elements with instantaneous coupling, and in recent years has been successfully extended to delay-coupled systems (Dhamala, Jirsa, and Ding, 2004; Choe et al., 2010).

Consider a network of $N$ identical elements described by the state vector $x_{i}(t)$, with $i=1, \ldots, N$ :

$$
\dot{x}_{i}(t)=f\left[x_{i}(t)\right]+\sum_{j=1}^{N} A_{i j} g\left[x_{j}(t-\tau)\right]
$$

The function $f$ represents the intrinsic dynamics of the nodes, and $g$ is the coupling function. Note that the delay time is assumed equal for all connections. The coupling matrix $A$ defines the connectivity of the network, with $A_{i j}$ representing 
the connection strength from node $j$ to node $i$. An invariant identical synchronization manifold exists if the row sum of the coupling matrix $\sigma_{j}=\sum_{i=1}^{N} A_{i j}$ is independent of the row (the value of $\sigma$ can then be interpreted as the coupling strength). The stability of a trajectory $x(t)$ along the synchronization manifold can be quantified by means of the variational equation

$$
\dot{\xi}(t)=D f[x(t)] \xi(t)+\gamma D g[x(t-\tau)] \xi(t-\tau),
$$

where $\xi(t)$ represents a perturbation away from $x(t)$. The factor $\gamma$ is a complex parameter that will take the values of the eigenvectors of the coupling matrix $A$. The master stability function is then defined as the maximum Lyapunov exponent of the variational equation (7) as a function of $\gamma$ and is usually denoted as $\lambda_{\max }(\gamma)$. The synchronized state is stable provided the master stability function is negative for all transverse eigenvalues of the coupling matrix (i.e., all eigenvalues excluding the one associated with perturbations within the synchronization manifold $\sigma$ ). Using this method, Kinzel et al. (2009) conjectured that the synchronized state becomes necessarily unstable for sufficiently large delay. The delay term in the variational equation (7) is not able to compensate for the destabilizing effect of the instantaneous term. Their work was supported by calculations on several specific chaotic maps and flows, but holding for any network with zero row sum, i.e., networks where the synchronized dynamics corresponds to the dynamics of an uncoupled unit. This conjecture was later confirmed and generalized by Flunkert et al. (2010), who showed that in the limit of large coupling delays the master stability function has two simple characteristics: it is symmetric with respect to $\gamma$ around the origin of the complex plane, and it increases monotonically with the radius in that plane, becoming positive beyond a critical radius $r_{0}$. This allows one to establish the general conditions for which identical synchronization is stable, on the basis of the properties of the coupling matrix and the dynamics around the synchronization manifold [as given by the variational equation (7)]. In particular, when the dynamics in the synchronization manifold is chaotic (which happens, provided $r_{0}$ is smaller than $|\sigma|$ ), the synchronized state is stable only if the largest eigenvalue of the coupling matrix is also smaller than $|\sigma|$ (and smaller than $r_{0}$, so that the master stability function is negative for all eigenvalues of the coupling matrix). Figure 32 shows the contour line of the master stability function $\lambda_{\max }=0$, computed numerically for a modified LK model of delay-coupled semiconductor lasers with increasing delays. The parameters of the model are such that the period of the relaxation oscillations is $T_{\mathrm{RO}} \approx 28$ in dimensionless units. The figure shows that for a coupling delay of order $T_{\mathrm{RO}}(\tau=20$, dot-dashed line) the contour line $\lambda_{\max }=0$ starts to become approximately circular, with the (chaotic) synchronized state being stable provided all eigenvalues of the coupling matrix fall within that circle. This provides a straightforward criterion to identify the stability of a laser network with a given coupling architecture, provided the network is homogeneous. The case of heterogeneous networks was recently analyzed by Nixon et al. (2012). In the case of a bipartite underlying graph, a master stability function reduction can be obtained for group synchronization (Sorrentino and Ott, 2007). In that way the stability problem

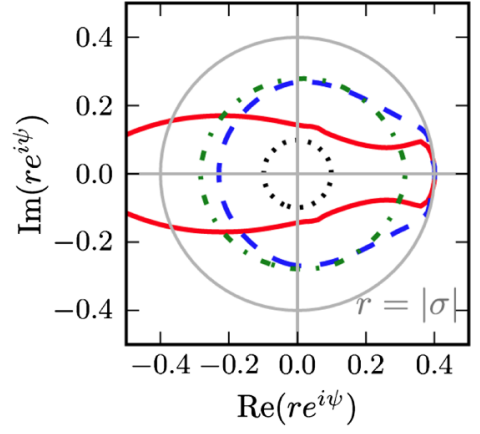

FIG. 32 (color online). Contour line of the master stability function, corresponding to a modified LK model of delaycoupled semiconductor lasers for increasing delay times: $\tau=1$ (solid line), $\tau=8$ (dashed line), $\tau=20$ (dot-dashed line), and $\tau=1000$ (dotted line). The light gray circle denotes the line $|\gamma| \equiv$ $r=\sigma$, which separates regions of different dynamics in the synchronization manifold: chaotic when the critical radius $r_{0}$ is inside the circle, and periodic or stationary otherwise. From Flunkert et al., 2010.

can be reduced to a low-dimensional form, independent of the choice of the (different) dynamical functions at the network nodes.

\section{F. Beyond stability}

The master stability function approach described in Sec. IV.E shows that the stability of the identically synchronized state in a laser network is completely determined by the eigenvalues of the coupling matrix. But even among networks with equal stability of the identically synchronous states, other properties of that regime can vary depending on the architecture of the network. Nishikawa and Motter (2010) showed that the coupling cost and the dynamical robustness depend in a nonmonotonic manner on the number of links of the network. An experimental investigation of this aspect was performed by Ravoori et al. (2011) and is shown in Fig. 33. They implemented a configurable network of four coupled optoelectronic oscillators. Each of these oscillators consisted of a semiconductor laser whose emitted light feeds a nonlinear loop formed by a Mach-Zehnder electro-optic intensity modulator and a digital signal processing board. The setup allowed them to test, in a systematic manner, the effect of the network topology on the different synchronization properties of the system. The top panel of Fig. 33 shows a collection of networks for which one connection is removed at a time, going from $m=12$ to $m=3$ links. The identically synchronized regime is stable for all these networks, provided the coupling strength lies in the correct synchronization range. However, the rate of convergence to the synchronous state (which is defined as the dynamical robustness of the network) changes noticeably as the number of links varies (bottom panel), with certain specific networks being optimal (denoted as such in the top panel), in the sense that all transverse eigenvalues of the coupling matrix are equal. For those networks the synchronization range and dynamical robustness are maximal, and the coupling cost is minimal (Nishikawa and Motter, 2010).

So far the network behavior seems to depend only on the eigenvalue spectrum of the coupling matrix. But even within 


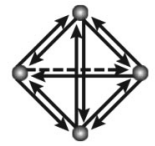

$m=12$

$\Lambda=\{0,4,4,4\} \quad \Lambda=\{0,3,4,4\}$ (optimal network)
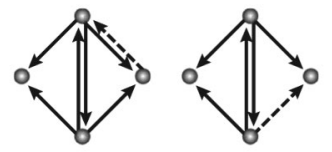

$m=7 \quad m=6$

$\Lambda=\{0,2,2,3\} \quad \Lambda=\{0,2,2,2\}$

(optimal network)
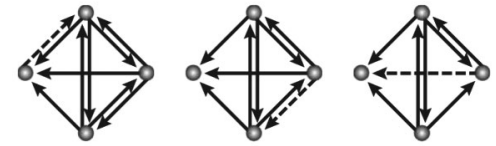

$m=10$

$m=9$

$m=8$

$\Lambda=\{0,3,3,3\} \quad \Lambda$
(optimal network)
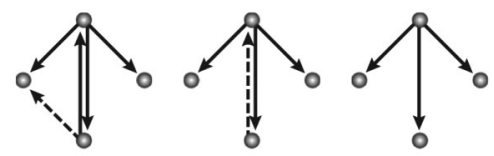

$m=5 \quad m=4 \quad m=3$

$\Lambda=\{0,1,2,2\} \quad \Lambda=\{0,1,1,2\} \quad \Lambda=\{0,1,1,1\}$

(optimal network)

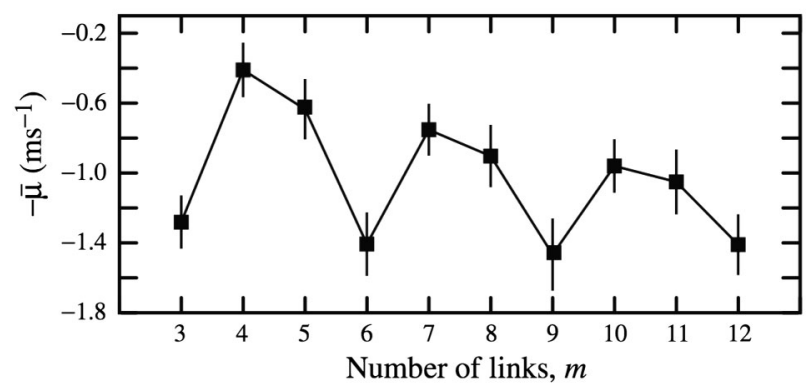

FIG. 33. Average convergence time (here denoted by $\bar{\mu}$, bottom panel) as a function of the number of links in a four-node network of optoelectronic oscillators. The top panel shows the different modules labeled with $m$, which denotes the number of links in each case. From Ravoori et al., 2011.

networks with identical eigenvalue spectra, substantial differences between their dynamical convergence to synchronization exist. In particular, Ravoori et al. (2011) distinguished two types of networks, which they call sensitive and nonsensitive, depending on the degeneracy of the eigenvectors of the coupling matrix. Networks whose coupling matrix has degenerate eigenvectors are sensitive, in the sense that they are more susceptible to the noise and mismatches that typically exist in real-life situations (in contrast to the ideal conditions of numerical simulations). Experimental realizations of both sensitive and nonsensitive networks were implemented by Ravoori et al. (2011) in the optoelectronic oscillator system. A comparison of the convergence to identical synchronization for the two network types is shown in Fig. 34. The networks are classified according to their geometric degeneracy $g_{d}$, defined as the largest number of different eigenvalues of the coupling matrix associated with the same eigenvector. The top panel of Fig. 34 shows four different networks with the same stability behavior (in fact they are all optimal, as given by their eigenvalue spectra) but different eigenvector degeneracy, measured by $g_{d}$. The bottom plot shows a comparison of the two networks with smallest and largest degeneracies. The plot shows that the nonsensitive networks have a much faster convergence to identical synchronization (given by a synchronization error that has reached the floor value, corresponding to experimental mismatches). Additionally, these networks exhibit the smallest degree of dynamical heterogeneity, since the time evolution of the synchronization error is very similar in all realizations of the dynamics. The situation contrasts strongly with the case of sensitive networks, which have a much
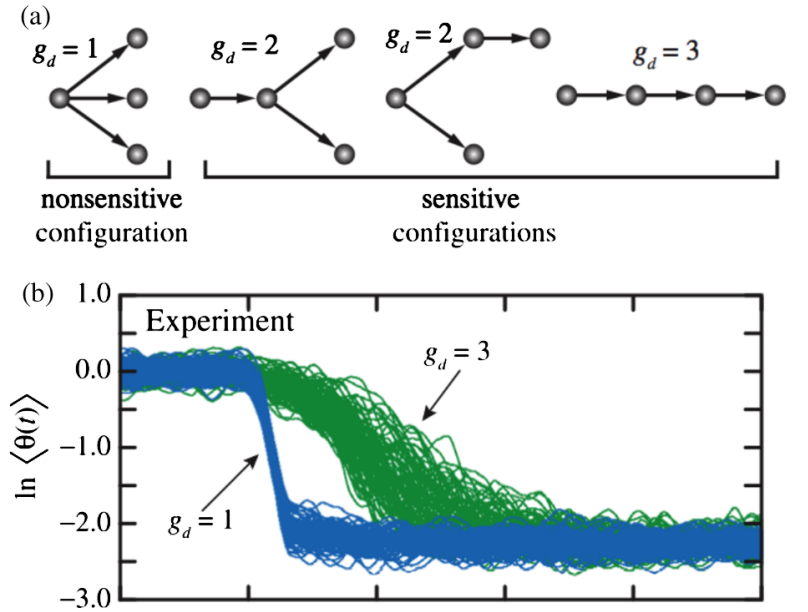

FIG. 34 (color online). Time evolution of the synchronization error [here denoted by $\theta(t)$, bottom panel] for two networks of four elements with different geometric degeneracy $g_{d}$. Dark gray lines correspond to nonsensitive networks with nondegenerate eigenvectors $\left(g_{d}=1\right)$, and light gray lines correspond to sensitive networks with $g_{d}=3$. The topologies of those two network motifs and of two other sensitive configurations are given in the top panel. From Ravoori et al., 2011.

slower and variable convergence to the identically synchronous state.

\section{G. Generalized synchronization and synchronization without correlation}

In this section, we go beyond the identical synchronization properties and address some aspects of generalized synchronization. As mentioned in Sec. III.A, the proof of generalized synchronization usually requires the auxiliary system approach. Nevertheless, correlation measurements are often being employed. Cross-correlation and mutual information quantifiers are standard methods to unveil connectivity information in complex networks. This information is crucial in many fields such as neuroscience, climate modeling, and ecological modeling, among others. The fundamental question of whether these two quantifiers are sufficient to detect the existence of generalized synchronization or not is still open. To tackle this problem, Van der Sande et al. (2008) studied a ring of $N$ unidirectionally delay-coupled lasers (see Fig. 35), as a paradigmatic example. Their study was based on numerical simulations of a modified version of the LK equations. The lasers, considered identical, were coupled via their emitted electromagnetic (EM) field. The total delay time $\tau_{c}$ was kept fixed independent of $N$, such that the coupling time between two consecutive lasers was, for a given $N$, $\tau_{c, N}=\tau_{c} / N$. Although the total delay was distributed among the $N$ elements, the same results hold for the case that all but one laser are coupled without delay and a single coupling delay $\tau_{c}$ between the first and last element.

From the time traces of the optical intensity of the lasers, no qualitative changes of the unstable dynamics is observed, when the number of lasers $N$ is increased. However, the power spectra and normalized intensity autocorrelation functions (AC) change significantly. In particular, the typical 


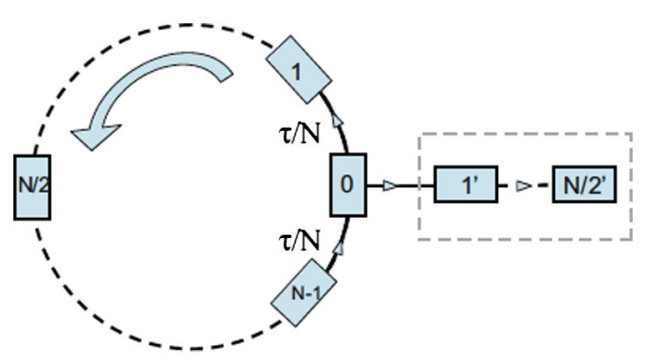

FIG. 35 (color online). A ring configuration of $N$ unidirectionally delay-coupled lasers which can optionally be coupled to a chain of lasers. From Van der Sande et al., 2008.

peaks in the $\mathrm{AC}$ at the delay time, and its harmonics, start to disappear as $N$ increases (Van der Sande et al., 2008) yielding a flat AC, except for the narrow peak located at $t=0$. Interestingly, it was found that the peak that appears at $t=N \tau_{c}$ in the AC of a single laser subject to delayed feedback appears at $t=\tau_{c}$ in the $\mathrm{AC}$ of any element in the ring of $N$ delay-coupled lasers. This means that the entire AC and power spectrum of a unidirectional ring of $N$ identical lasers can be deduced solely from the AC of the single laser with delayed optical feedback. Since the heights of the peaks at $t=N \tau_{c}$ in the AC of a single laser with delayed feedback exponentially decay as $N$ increases, the AC peak at $t=\tau_{c}$ in a ring of unidirectionally coupled lasers eventually disappears for large enough $N$. In the same manner, any signature of the delay time disappears from the power spectrum. Additionally, the cross-correlation function between elements $N / 2$ and 0 also decays (exponentially) as the number of lasers increases, as illustrated by the squares in Fig. 36. The same occurs for the mutual information in generic motifs of delay-coupled elements (Soriano et al., 2012).

The negligible correlation (and mutual information) observed between the most distant elements in the ring raises the question of whether these elements are still generally synchronized or not. To answer this, the auxiliary system approach (described in Sec. III.A) can be implemented (Abarbanel, Rulkov, and Sushchik, 1996). To this end, a chain, identical to the one that mediates between element 0 to $N / 2$, is attached to element 0 (see Fig. 35). When comparing the time traces of elements $N / 2$ and $N^{\prime} / 2$ they turn out to be identical, i.e., identical synchronization is established between the two semiconductor lasers. Figure 36 shows the maximum of the cross-correlation function between lasers $N / 2$ and 0 and $N / 2$ and $N^{\prime} / 2$. It is worth highlighting that the identical synchronization between $N / 2$ and $N^{\prime} / 2$, reached through an uncorrelated mediating signal, necessarily implies that the coupling signal is generally synchronized to the original one.

The existence of a generally synchronized state with negligible correlation (or mutual information) measures has been recently extended to more general configurations in the framework of simple network motifs (Soriano et al., 2012).

In conclusion, we have seen in this section that laser networks provide a rich test bed for complex dynamics. Zero-lag and sublattice synchronization have been observed in laser systems, and the conditions for the existence of such regimes have been studied. In the case of small networks (or motifs), the coupling symmetries strongly influence the collective

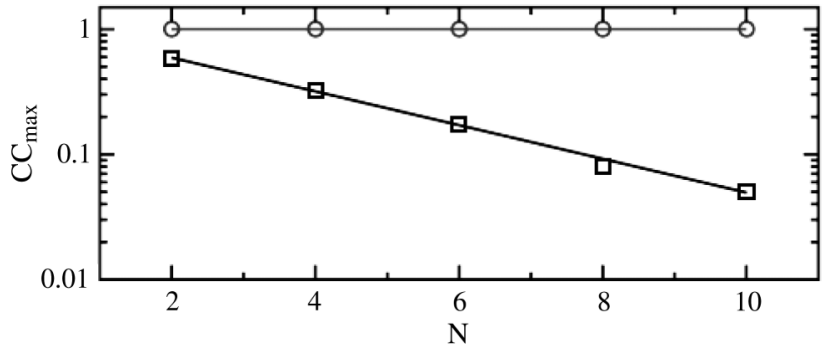

FIG. 36. Maximum value of the cross correlation (CCmax) between the semiconductor laser $N / 2$ and 0 (squares) and between $N / 2$ and $N^{\prime} / 2$ (circles) vs the number of lasers $N$. The solid line is an exponential fit. From Van der Sande et al., 2008.

behavior of the system. In the case of larger networks, the influence of the coupling architecture enters the system via both the spectrum of eigenvalues of the coupling matrix and the degeneracy of its eigenvectors. The former determines in a unique manner the stability of the synchronized state through the master stability approach. The latter determines the sensitivity of the network to external imperfections, and the convergence to the synchronized state, even when the stability properties of the networks are identical to each other. We also described a scenario for the emergence of generalized synchronization in the absence of correlation between the network elements.

\section{APPLICATIONS OF DELAY-COUPLED LASERS}

In recent years, laser instabilities are in many cases considered less as a nuisance that have to be suppressed or controlled but instead are seen as a valuable resource that can be exploited. In semiconductor lasers, these instabilities often arise as a result of external perturbations (Krauskopf and Lenstra, 2000). As introduced in Sec. II, delayed selffeedback gives rise to a rich scenario of instabilities and complex dynamical behavior. Such behavior has been employed in novel and unexpected photonic applications. In particular, photonic integrated sources promise significant contributions to the practical use of complex dynamics (Yousefi et al., 2007; Argyris et al., 2008).

A single laser with feedback can be employed as a versatile broadband source in a wide variety of photonic applications [see, e.g., Ohtsubo (2008) and In, Longhini, and Palacios (2009)]. In this section, we first cover the utilization of a laser with delayed feedback as a key element in the generation of laser light with tunable coherence length (Peil, Fischer, and Elsäßer, 2006), chaotic LIDAR applications (Lin and Liu, 2004), and the generation of random bits at $\mathrm{GHz}$ speed (Uchida et al., 2008).

Several of these applications rely on the generation of chaotic broadband spectra due to the presence of a relatively strong delayed self-feedback. Laser chaos exhibits several noiselike features, such as broadband spectra and a rather unpredictable output. However, chaos has a deterministic origin and, as such, it is fundamentally different to stochastic dynamics. Importantly, this deterministic nature of laser chaos allows for the synchronization of two or more delaycoupled semiconductor lasers (Mirasso, Colet, and GarciaFernandez, 1996). 
The synchronization of delay-coupled semiconductor lasers has paved the way for the implementation of successful photonic applications (Donati and Mirasso, 2002). A prominent example is the laser-based chaos communication field [see, e.g., Argyris et al. (2005)]. The main goal of this research field is to provide an extra level of security at the physical layer to the communication protocol.

Additionally, it is worth emphasizing a novel aspect of laser-based chaos communications, namely, public key exchange. Most cryptographic methods rely on the exchange of a private key prior to communication. Therefore, methods to generate a secret key over a public channel are desired. Recently, the synchronization of mutually coupled lasers was suggested as a way to generate a private key in the two agents of a communication link in the framework of public channel cryptography (Klein, Gross, Kopelowitz et al., 2006; Kinzel and Kanter, 2008; Vicente, Mirasso, and Fischer, 2007).

Finally, we highlight the intriguing similarities between delay-coupled lasers and neuronal systems with respect to the emergence of collective dynamics and synchronization phenomena (Fischer et al., 2006). These similarities have also initiated the development of bioinspired photonic information processing applications (Appeltant et al., 2011; Larger et al., 2012). Thus, fruitful cross fertilization between photonics and neuroscience is possible and first success is already visible.

\section{A. Laser source with tunable coherence length}

The generation of highly coherent light is mandatory for several applications such as coherent optical fiber communications (Yamamoto and Kimura, 1981) and optical metrology (Kowarschik, Tschudi, and Wang, 2001). Semiconductor lasers are ubiquitous in these applications, due to their attractive properties including small dimensions and high wall-plug efficiency. In some applications, however, incoherent light is required to avoid interference effects of coherent light. Examples of such applications are rainbow refractometry (Peil et al., 2006), fiber-optic gyroscopes (Donati, 2004), and coherence tomography (Huang et al., 1991).

As described in Sec. II.D.1, semiconductor lasers subject to delayed optical feedback exhibit interesting properties. For instance, their stability properties allow for two kinds of opposite behavior, depending on the feedback strength (Tkach and Chraplyvy, 1986). Lasers operate in a stable single mode in the limit of very weak or strong feedback. In contrast, the lasers typically emit in several longitudinal and/or external-cavity modes for intermediate feedback strengths. Consequently, the optical linewidth of the laser very much depends on the operating conditions. In the $\mathrm{CC}$ regime, the optical linewidth can be broadened beyond $10 \mathrm{GHz}$. An example of the different optical spectra that can be obtained in a setup comprising a semiconductor laser subject to delayed optical feedback is given in Fig. 37. The linewidth of the solitary laser is in the $\mathrm{MHz}$ range, while the linewidth in the case of intermediate feedback lies in the multi-GHz range.

The coherence length of the laser quantifies the optical path difference over which self-interference fringes can be obtained. It was shown that the nonlinear dynamical properties of semiconductor lasers subject to delayed feedback allow for controlled adjustment of the coherence length in a

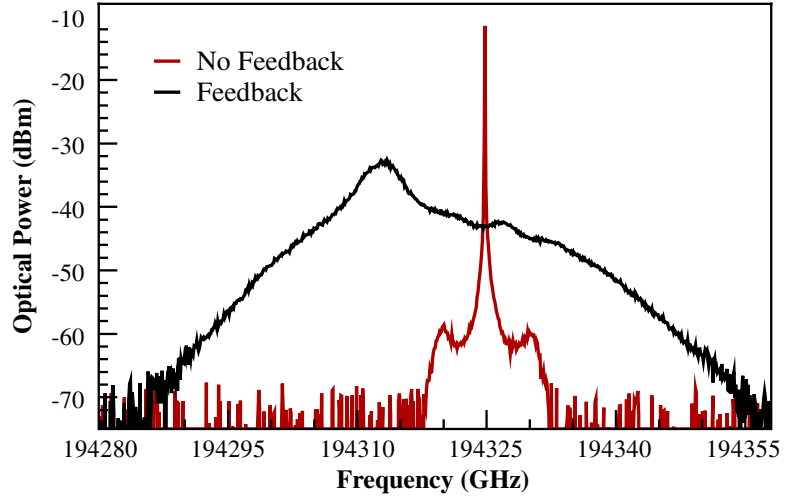

FIG. 37 (color online). Measured optical spectra of a semiconductor laser (light gray) without feedback and (black) with intermediate feedback strength. Details of the experimental setup can be found in Soriano, Zunino et al. (2011).

wide tuning range (Peil, Fischer, and Elsäßer, 2006). Importantly, the coherence length can be tuned by changing a single feedback parameter, e.g., the feedback phase or the feedback strength. In particular, a smooth change in the feedback phase allows for a complete span of the coherence length in a short-cavity configuration, thus operating in the SDR (Peil, Fischer, and Elsäßer, 2006). At the same time low intensity noise in the low-frequency region can be achieved with this setup. In a setup operating in the LDR, the feedback strength is the tuning parameter (Wang et al., 2009).

The visibility obtained from a Michelson interferometer is often used to quantitatively characterize the coherence properties of the laser subject to delayed feedback. The coherence length of the laser is defined as the distance at which the visibility function has decayed to $1 / e$ of its maximum value. The tunability of the coherence length previously reported is in the range of $\sim 130 \mu \mathrm{m}$ to $\sim 8 \mathrm{~m}$ in the short-cavity setup, when the feedback phase is varied via piezoelectric elements (Peil, Fischer, and Elsäßer, 2006), and in the range $\sim 100 \mu \mathrm{m}$ to several meters (out of measurement range) in the longcavity setup when the feedback strength is varied via a halfwave plate (Wang et al., 2009). The dramatic decrease of the coherence length for increasing feedback strength is depicted in Fig. 38, in which the different temporal dynamics associated with selected feedback strengths are shown in the insets. An increase in the complexity of the temporal waveform is linked to a decrease in the coherence length of the laser.

A semiconductor laser with tunable coherence length is a versatile tool that can be helpful in a large variety of applications. A first proof of principle was introduced in a rainbow refractometry experiment (Peil et al., 2006). In this realization, the size of a droplet was determined by analysis of the primary rainbow scattering. For coherent illumination, unwanted interference ripples were detected, while for incoherent illumination a smooth distribution was obtained. Thus, the accuracy of the measurement is significantly improved by the use of incoherent laser light, highlighting the potential of this simple setup.

\section{B. Chaotic LIDAR and remote sensing applications}

Light detection and ranging is an optical remote sensing technique that allows one to measure the distance to a target 


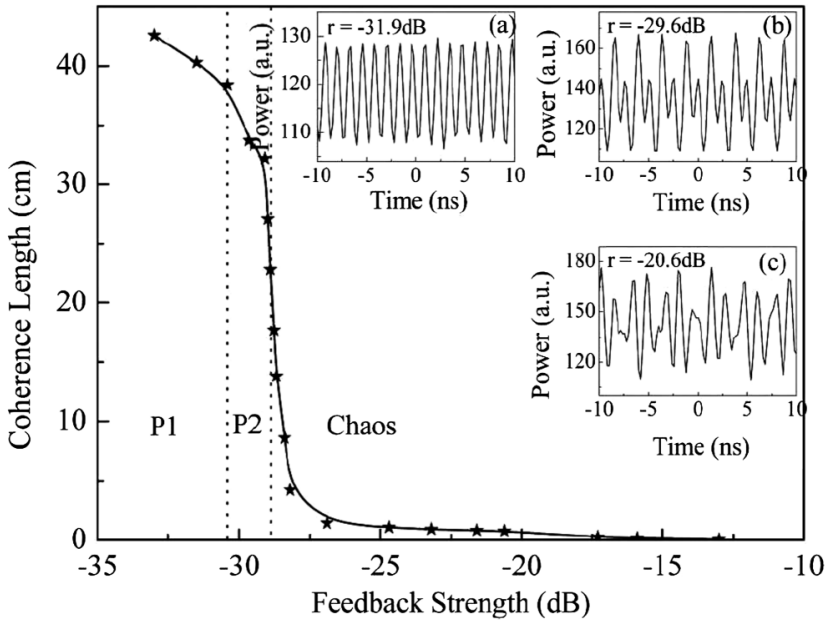

FIG. 38. Coherence of a semiconductor laser with delayed optical feedback as a function of the feedback strength. From Wang et al., 2009.

by detecting the back reflections from it. It was demonstrated that nonlinear dynamical properties of semiconductor lasers can be exploited for remote sensing applications. A LIDAR system that uses a chaotic laser as a source was proposed and studied by Lin and Liu (2004).

Laser LIDAR systems are mostly based on two types of approaches to properly measure the distance to a target. They use either pulsed lasers or modulated continuous-wave lasers. In the pulse technique, the distance is measured directly from the delayed back reflections of the pulses yielding a typical resolution on the order of meters (Pearson et al., 2002). The resolution of the continuous-wave LIDARs is determined by the bandwidth of the modulated source. The resolution of a randomly modulated $\mathrm{cw}$ LIDAR is of the order of tens of meters (Takeuchi et al., 1983).

A LIDAR system that utilizes the output of a chaotic laser as a source has a higher resolution than its standard counterparts. With the bandwidth of a chaotic laser easily being broader than $15 \mathrm{GHz}$, centimeter resolution can be achieved. Such large bandwidths can be obtained when a semiconductor laser is subject to external perturbations such as optical injection (Wieczorek, Krauskopf, and Lenstra, 1999), optical feedback (Lenstra, Verbeek, and den Boef, 1985), or optoelectronic feedback (Tang and Liu, 2001b). Numerical and experimental studies suggest that chaotic LIDAR needs to be operated in a regime of a flat and smooth spectrum in order to show good performance (Lin and Liu, 2004). A similar chaotic broadband source was also suggested to improve the resolution of optical time domain reflectometry systems (Wang, Wang, and Wang, 2008).

The working principle of chaotic LIDAR is sketched in Fig. 39, in which a distant target is illuminated by the output emission of a laser operating in the chaotic regime. The relative distance to the object can be measured by calculating the cross correlation between the light emitted by the laser and the light reflected from the target. An example of target detection is shown in Fig. 40 for an experiment in which an object was displaced from its original position by about $50 \mathrm{~cm}$. The displacement is properly

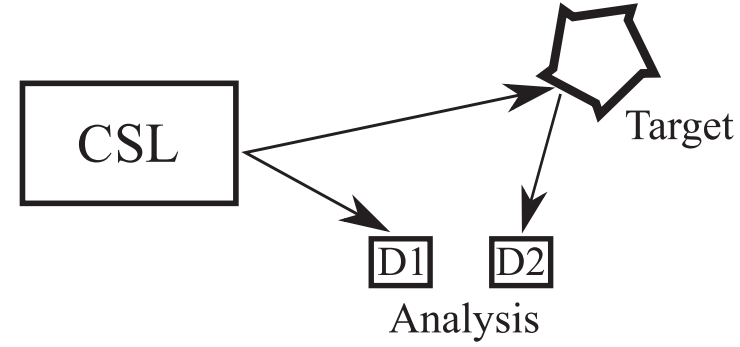

FIG. 39. Schematic setup of the LIDAR system with a chaotic semicondutor laser (CSL) as a source. D1 and D2 denote the photodetectors.

captured by the measurement system providing a resolution of $3 \mathrm{~cm}$ which is limited by the detection apparatus.

One limitation of resolution is determined by channel noise. Therefore, the influence of channel noise on the detection performance of chaotic LIDARs was investigated by $\mathrm{Wu}$, Liao, and Lin (2010). Channel noise in a real environment is a combination of atmosphere disturbances, which can be modeled as additive white Gaussian noise and random phase noise sources on the amplitude and phase of the signal waveforms. The influence of undesired noise can be minimized by further exploiting the chaotic properties of the emitted light. A system with two synchronized chaotic LIDARs was proposed in which one of the lasers acts as a transmitter and the other one as a receiver. The noise-contaminated reflected chaotic signal can be coupled into the receiver laser, achieving synchronization of the chaotic light. The receiver laser then reproduces the original chaotic waveform from the transmitter laser, filtering to a large extent the channel noise, an effect called chaos-pass filtering (Fischer, Liu, and Davis, 2000). Employing an open-loop configuration, generalized synchronization is established. For an optimized coupling strength, that depends on the noise level, optimal performance was obtained (Wu, Liao, and Lin, 2010).

Delayed optical feedback to semiconductor lasers has also been successfully applied in a variety of remote sensing

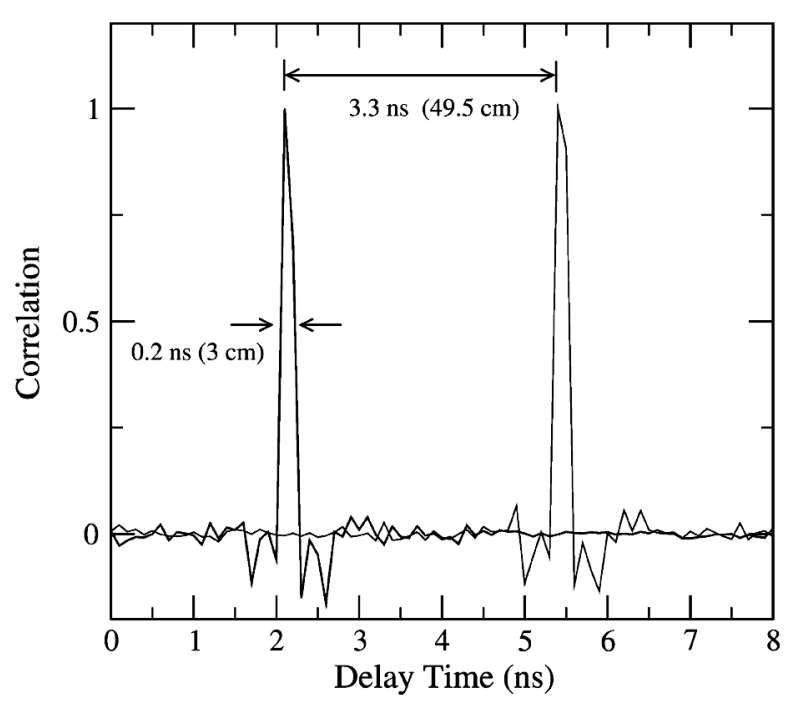

FIG. 40. Cross-correlation traces of a target moving $50.0 \mathrm{~cm}$ in the line of sight. From Lin and Liu, 2004. 


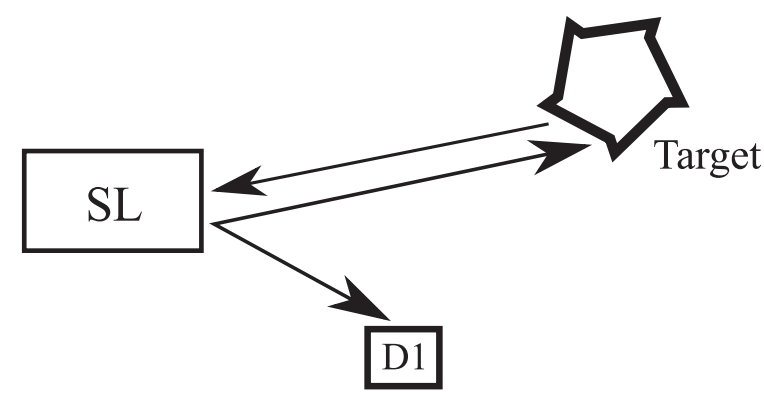

FIG. 41. Schematic setup of a remote sensing system, exploiting the self-mixing effect of a semicondutor laser (SL). D1 denotes the photodetector.

applications, exploiting the self-mixing effect (Giuliani et al., 2002). In the self-mixing effect, illustrated in Fig. 41, the reflection from the remote target is fed back into the laser, modulating its output power. Thus, the system operates as an interferometer, capable of measuring displacements without ambiguity (Donati, Giuliani, and Merlo, 1995), velocities (Shinohara et al., 1986; Albert et al., 2004), or vibrations (Scalise et al., 2004). It was shown that the self-mixing technique is a powerful tool, based on a simple setup.

A nonlinear feedback loop incorporating a wave chaotic system was recently employed for a position sensing system (Cohen et al., 2011). In a wave chaotic cavity, EM waves fill the entire cavity through many multipath reflections. The combination of nonlinear delayed-feedback systems and wave chaotic cavities gives rise to high sensitivity and subwavelength accuracy, overcoming even the diffraction limit. So far the effect was demonstrated with radio-frequency waves, with a clear potential to be extended into the photonics domain.

\section{Random bit sequence generation}

The generation of random bit sequences is required in several key digital technologies, including authentication and encryption protocols, on-line gaming, lotteries, optical communications, and Monte Carlo simulations (Ferguson, Schneier, and Kohno, 2010; Uchida and Atsushi, 2012). Random bit generators (RBGs) are either built upon physical entropy sources or use a deterministic algorithm based on a random seed, the so-called pseudorandom bit generators. Various physical processes, such as electronic noise or photonic noise (Holman, Connelly, and Dowlatabadi, 1997; Williams et al., 2010), were suggested as a source to generate random bits. In addition, quantum RBGs promise truly random bit sequences (Gabriel et al., 2010). However, the bit rate provided by quantum physical sources does not meet the requirements of modern data rates yet. In turn, pseudorandom

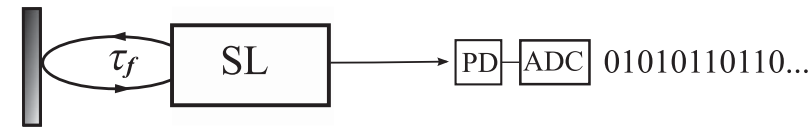

FIG. 42. Schematic representation of the generation of random bits (RBG) with a semicondutor laser (SL) subject to delayed feedback. PD and ADC denote the photodetector and the analogto-digital converter, respectively. bit generators have a high bit rate, but are vulnerable if the original seed can be guessed. A number of photonic implementations based on chaotic semiconductor lasers were recently proposed to overcome these limitations. Uchida et al. (2008) were the first to announce 1.7 gigabits per second $(\mathrm{Gb} / \mathrm{s})$ RBG based on two chaotic semiconductor lasers, whereas Reidler et al. (2009) were the first to announce $12.5 \mathrm{~Gb} / \mathrm{s}$ RBG based on a single chaotic laser.

As illustrated in Fig. 42, the idea behind the laser-based implementations is to digitize a chaotic analog signal (Murphy and Roy, 2008; Uchida et al., 2008; Reidler et al., 2009), taking advantage of the inherent noise in combination with chaos-induced decorrelation of the trajectory as the basis for the generation of independent bits (Mikami et al., 2012). Semiconductor lasers subject to delayed optical feedback can produce strongly diverging chaotic trajectories, thus making high bit rates possible (Argyris, Deligiannidis et al., 2010; Hirano et al., 2010; Kanter, Aviad et al., 2010; Oliver et al., 2011). The chaotic signals typically consist of irregular picosecond pulses (Fischer, van Tartwijk et al., 1996).

Digitization procedures are necessary to achieve a sequence of random bits from an analog dynamical system. These procedures include a variety of postprocessing methods, which are ideally kept to a minimum, not only to harness most of the original information, but also to avoid a slowdown of the process when implemented in real time. The pioneering work of Uchida et al. (2008) reported real-time generation of random bits at a rate up to $1.7 \mathrm{~Gb} / \mathrm{s}$. This was achieved by sampling the fluctuating optical output of two chaotic laser systems with one-bit analog-to-digital converters (ADC). The generation rate was at that time an order of magnitude faster than for previously reported RBGs. In the work of Uchida et al. (2008) the only required digital postprocessing was the combination of the binary signals by a logical exclusive-OR (XOR) operation.

Subsequent works tackled the ongoing challenge of increasing the bandwidth of laser-based RBGs and the development of advanced postprocessing techniques to maximize the bit generation capacity. A number of studies focused on the enhancement of the original bandwidth of the chaotic signal by using optically coupled semiconductor lasers (Hirano et al., 2010). Several studies concentrated on the optimization of the digital postprocessing techniques (Reidler et al., 2009; Kanter, Aviad et al., 2010). Often the chaotic dynamics of the semiconductor lasers is sampled with 8-bit ADC and postprocessed off-line to generate random bit sequences. Specifically, Reidler et al. (2009) achieved a potential $12.5 \mathrm{~Gb} / \mathrm{s}$ generation rate by computing the first order derivative of the acquired 8-bit samples and keeping the 5 least significant bits of the chaotic fluctuations of a single laser with feedback. Even faster bit rates of $300 \mathrm{~Gb} / \mathrm{s}$ were reported by computing higher order derivatives (Kanter, Aviad et al., 2010). Using bandwidth enhanced chaotic lasers, the potential for a random bit rate of $75 \mathrm{~Gb} / \mathrm{s}$ was demonstrated by performing a bitwise XOR operation of the acquired samples (8-bit resolution) and keeping the 6 least significant bits (Hirano et al., 2010). The importance of discarding the most significant bits is illustrated in Fig. 43 for an experiment reported by Oliver et al. (2011). The 

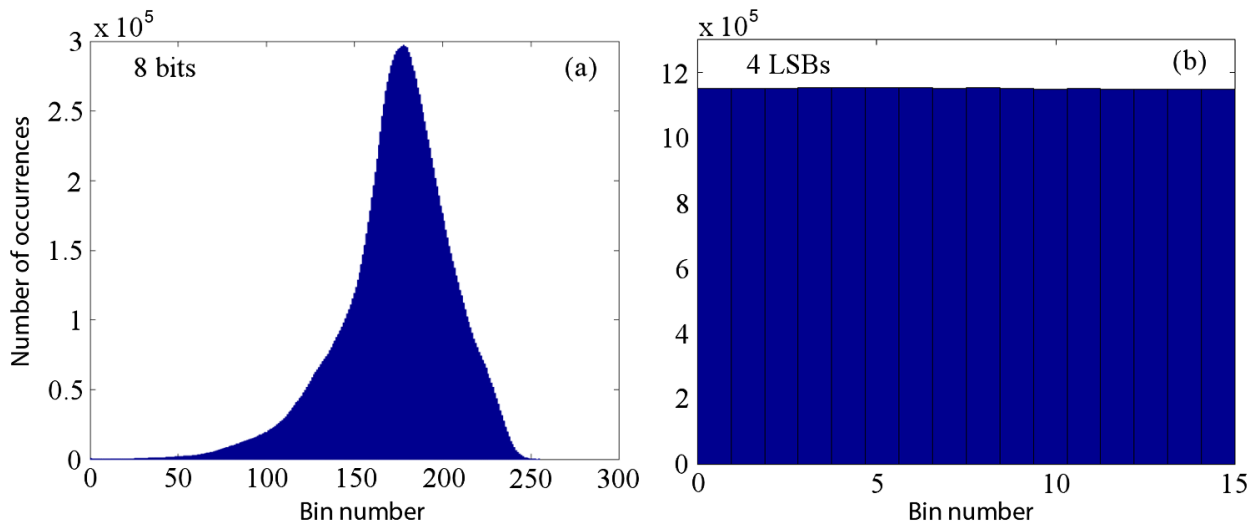

FIG. 43 (color online). Bin distribution of the amplitude fluctuations of a semiconductor laser with optical feedback with (a) 8 bits ADC resolution and (b) keeping the four least significant bits (LSBs). Details of the experimental setup can be found in Oliver et al. (2011).

histogram of the acquired chaotic dynamics is plotted with the full 8-bit ADC resolution, Fig. 43(a), and with the 4 least significant bits, Fig. 43(b). The distribution of the fully resolved chaotic signal is inhomogeneous, while the histogram of the data with only the 4 least significant bits is flat and hence more suitable for the generation of random bits.

In the system of a laser with optical feedback, the externalcavity length, the injection current, and the feedback strength are three crucial system parameters that need to be carefully adjusted. Therefore, RBGs based on a thresholding principle might suffer from undesired experimental drifts (Uchida et al., 2008). In these cases, a real-time adaptive control of the ratio between 0's and 1's in the sequences of generated random bits turns out to be a practical way to maintain long term randomness (Uchida and Atsushi, 2012). In contrast, the influence of small experimental drifts can be eliminated by using more elaborate postprocessing methods, e.g., by computing the derivate of the intensity distribution (Reidler et al., 2009).

Robust and compact RBGs are desirable for practical implementations. This was realized using a semiconductor laser subject to polarization-rotated optical feedback, based on standard, fiber-based telecommunication components (Oliver et al., 2011). To be compatible with real-time operation, postprocessing requirements were minimized. A systematic study of the influence of the underlying dynamics in the generation of random bits was performed (Oliver et al., 2011). In addition, an integrated compact real-time random bit generator was reported with a rate of $140 \mathrm{~Gb} / \mathrm{s}$ (Argyris, Deligiannidis et al., 2010).

Parallel to the efforts invested at improving RBGs based on semiconductor lasers with feedback, significant advances on RBGs based on amplified spontaneous emission noise sources were recently reported (Williams et al., 2010). In Williams et al. (2010), the amplified spontaneous emission produced in a fiber amplifier was processed using only threshold detection and XOR decorrelation techniques to achieve a rate of $12.5 \mathrm{~Gb} / \mathrm{s}$ random number generation.

The randomness of the generated bit sequences is usually tested using standard statistical test suites that are publicly available. In particular, the National Institute of Standards and Technologies (NIST) provides such a suite for random and pseudorandom number generators for cryptographic applications (Rukhin et al., 2010). It consists of 15 tests that are recommended to be run with 1000 sequences of length of 1 Mbit each. A detailed description of these tests and a guide to use the software can be found in Rukhin et al. (2010). It is worth mentioning that tests cannot ensure randomness with finite length sequences. Complementary test suites include Diehard and Dieharder, providing further statistical tests for determining the quality of RBGs (Marsaglia, 1995).

Finally, an interesting issue is to what extent random bit generators based on delay-coupled chaotic lasers can be synchronized and therefore simultaneously generated at different sites. Recently, a numerical method to generate random bits in a synchronized manner was proposed (Kanter, Butkovski et al., 2010). A first experimental implementation was reported by Yoshimura et al. (2012) with bit rates of $2 \mathrm{Mb} / \mathrm{s}$. By using lasers with short feedback loops and reaching fast resynchronization times, the bit generation rate might be increased beyond $1 \mathrm{~Gb} / \mathrm{s}$.

\section{Chaos communication and chaos key distribution}

The seminal papers of Pecora and Carroll (1990) and Winful and Rahman (1990) created strong interest in the study of the synchronization of chaotic dynamical systems. This field started to develop in the early 1990s and has been of great relevance since then. The first experimental demonstration of chaos synchronization was performed using electronic circuits (Pecora and Carroll, 1990, 1991). The potential of synchronized coupled chaotic lasers was soon recognized and first experimental demonstrations appeared for $\mathrm{CO}_{2}$ and solid-state lasers (Roy and Thornburg, 1994; Sugawara, Tachikawa, and Tsukamoto, 1994), and later for semiconductor lasers with optical feedback (Sivaprakasam and Shore, 1999).

The possibility of using synchronization of chaos in encrypted communication was suggested by Cuomo, Oppenheim, and Strogatz (1993). Soon after, AnnovazziLodi, Donati, and Sciré (1996) and Mirasso, Colet, and Garcia-Fernandez (1996) transferred the concept to generalized synchronization of chaotic semiconductor lasers and demonstrated its potential using numerical simulations. The first practical application of chaotic lasers to encrypted 
communication was demonstrated using erbium-doped fiber ring lasers (Van Wiggeren and Roy, 1998) and semiconductor lasers (Goedgebuer, Larger, and Porte, 1998; Fischer, Liu, and Davis, 2000; Sivaprakasam, 2000; Liu et al., 2001; Kusumoto and Ohtsubo, 2002). The large bandwidth of semiconductor lasers and its versatility lie at the heart of the success of encrypted communications using these lasers. Many details on this application can be found in Uchida et al. (2005), Ohtsubo (2008), and Uchida and Atsushi (2012).

After about ten years of research (Uchida et al., 2005), a field experiment in the metropolitan area network of Athens demonstrated chaos-based optical encryption in a standard network infrastructure (Argyris et al., 2005). This demonstration was a real breakthrough for a number of reasons, including propagation over long distances (more than $100 \mathrm{~km})$, high transmission rates $(>1 \mathrm{~Gb} / \mathrm{s})$, and low BERs $\left(<10^{-6}\right)$.

As discussed in Sec. III, different architectures have been employed in the synchronization of two chaotic semiconductor lasers. In most studies of semiconductor lasers used in chaos communications, the coupling between the lasers, and thus the information transmission, has been unidirectional. The message is then encoded in one of the lasers, being known as the transmitter or drive laser, and recovered in the respective other one, being known as the receiver or response laser. The typical schematic arrangement of two unidirectionally coupled semiconductor lasers is shown in Fig. 44. The output of the drive laser that operates in a chaotic regime due to delayed feedback is injected into the response laser. If the response laser is not subject to delay feedback itself, the scheme is called an open loop. In contrast, if the response laser is also subject to feedback it is referred to as a closedloop scheme (Vicente, Perez, and Mirasso, 2002).

The message can be encoded in different ways into the chaotic carrier. For instance, it can be encoded by directly modulating the injection current of the laser or by externally modulating the laser output. The encoded message, which represents a perturbation to the receiver laser, can then be recovered by comparing the incoming signal and the response waveform of the receiver (Fischer, Liu, and Davis, 2000; Sivaprakasam, 2000; Abarbanel et al., 2001). This is known as the chaos-pass filtering technique (Fischer, Liu, and Davis, 2000). It is important to note that although in principle also identical synchronization might be employed (Vicente, Perez, and Mirasso, 2002; Li, Liao, and Wong, 2004), it turned out that generalized synchronization is more robust and therefore has been used in almost all demonstrations based on chaotic semiconductor lasers. Nevertheless, subtraction of incoming signal and response waveform can be used for message extraction, since the generalized synchronization between transmitter and receiver exhibits sufficiently high correlation. Interestingly, a scheme that would benefit from the combination of generalized and identical synchronization is, in theory, possible (Pisarchik and Ruiz-Oliveras, 2010).

The complete process of message encoding and decoding is illustrated in Fig. 44, where the message is encrypted by modulating the output emission of the transmitter laser and recovered at the receiver side by reconstructing only the chaotic waveform of the incoming signal. In the case of the closed-loop scheme, the message could also be encoded by

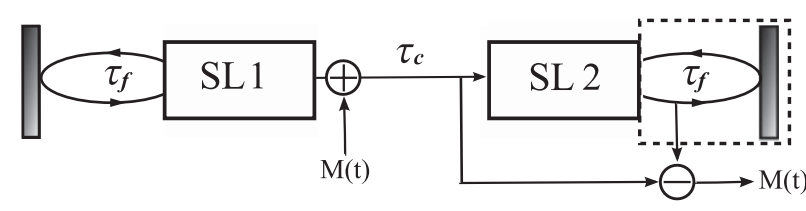

FIG. 44. Schematic arrangement of chaos communication in a setup with two unidirectionally coupled semiconductor lasers. The external cavity of the receiver laser is enclosed by a dotted box to distinguish between the open-loop scheme (no external cavity) and the closed-loop scheme (with external cavity for the receiver laser). The message $M(t)$ is encoded into the chaotic carrier at the transmitter and recovered at the receiver side.

varying the optical phase of the external cavity in the transmitter laser. It was observed that the receiver laser only synchronizes to the transmitter laser if the phases of the two external cavities are matched (Heil et al., 2002). The information can then be recovered as two different states of synchronization, i.e., high correlation or low correlation between transmitter and receiver laser.

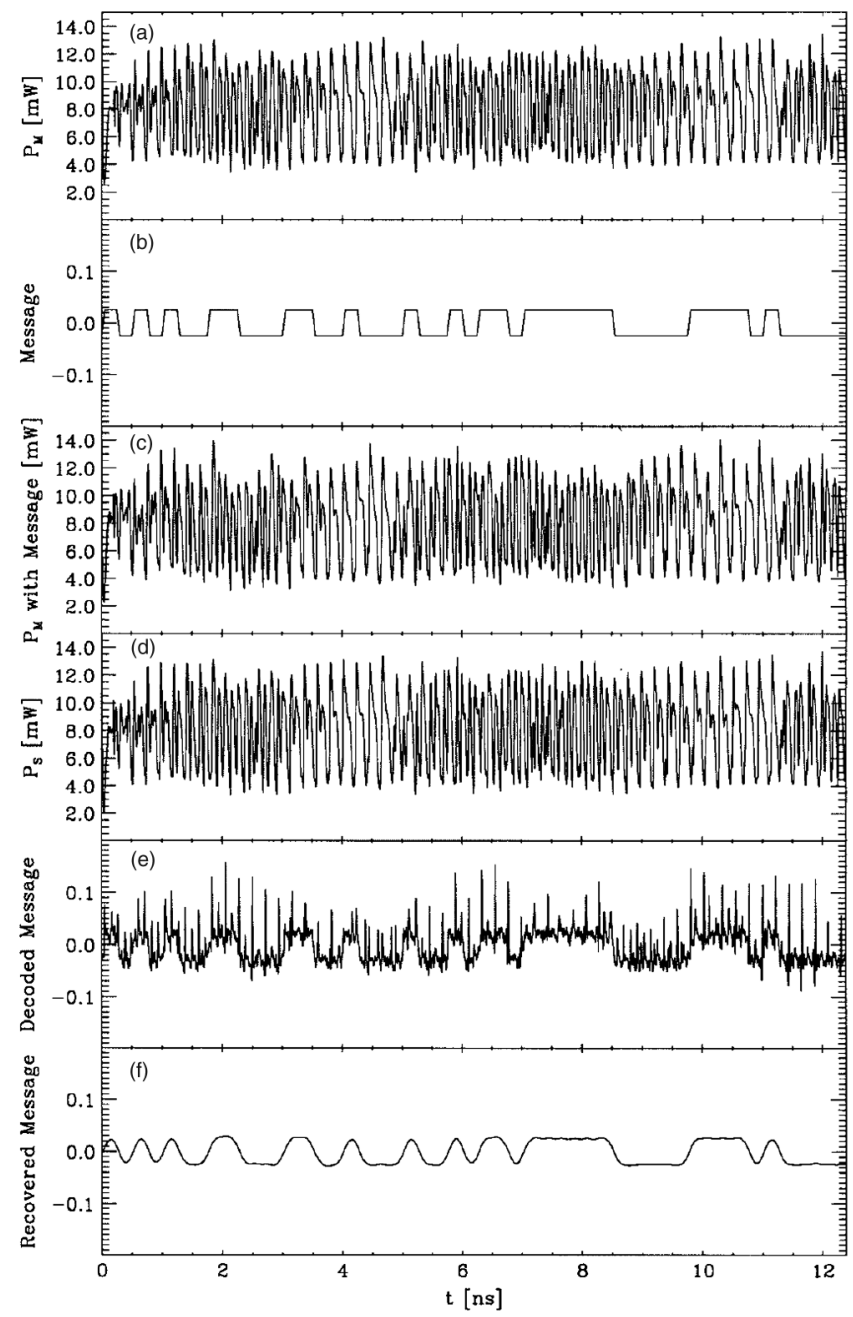

FIG. 45. Dynamical evolution of the output intensities of unidirectionally coupled transmitter and receiver lasers. (a) Typical laser output from the transmitter, (b) encoded digital message at a bit rate of $4 \mathrm{~Gb} / \mathrm{s}$, (c) transmitter output after encoding the digital signal, (d) output of the receiver, (e) decoded message, and (f) decoded message after filtering. From Sanchez-Diaz et al., 1999. 
An example of a chaos-based message transmission is demonstrated via numerical simulations in Fig. 45. A digital message is hidden into the chaotic carrier by modulating the intensity of the transmitter laser. The message is recovered at the receiver via the chaos-pass filtering technique. The signal-to-noise ratio of the recovered message can usually be improved by low-pass filtering after reconstruction; see Figs. 45(e) and 45(f).

The polarization state of the light fed back into the laser also plays a significant role in the dynamics of semiconductor lasers subject to feedback. A secure communication scheme that exploits the particular properties of polarization-rotated feedback was suggested by Rogister et al. (2001), in which they concluded that this scheme does not require fine-tuning of the laser optical frequencies if the polarization-rotated feedback can be treated as an incoherent effect. However, this conclusion is no longer valid when the polarizationrotated feedback has to be treated as a coherent effect (Heil, Uchida et al., 2003). The polarization state of light also plays an important part in the properties of VCSELs, and thus light polarization must be taken into account in chaos communication schemes using these types of lasers (Lee, Hong, and Shore, 2004). Interestingly, the rich polarization properties of VCSELs can also be used in high-speed chaos-based communications (Scire et al., 2003; Hong et al., 2009).

Most studies consider the synchronization of two coupled identical semiconductor lasers. However, in practice, the manufacturing process of these lasers is not fully reproducible, resulting in mismatches of the expected laser parameter values. High-quality synchronization requires the coupled lasers to be equal within a tolerance range typically smaller than a few percent in their corresponding parameter values (Sanchez-Diaz et al., 1999). The cross-correlation measure between the coupled lasers degrades rapidly for larger parameter mismatches (Vicente, Perez, and Mirasso, 2002). Identical synchronization is, however, a lot more sensitive to parameter mismatch than generalized synchronization. The encoded message cannot be properly recovered at the receiver laser side, if chaos synchronization is lost. Some practical realizations have overcome some of these problems by using a relatively large amplitude of the message. However, the security of the communication link is compromised, if the amplitude of the encoded message is too large (Soriano, Colet, and Mirasso, 2009). The strong dependence of the synchronization on the laser parameters is in turn an advantage from the security point of view, since an eventual eavesdropper would need very particular hardware to extract a message hidden within the chaotic carrier.

Semiconductor lasers have been preferably used in chaos communication schemes due to their large modulation bandwidth. The maximum transmission bit rate is mainly limited by the relaxation oscillation frequency, which is typically in the GHz range. Besides the optical feedback schemes, optoelectronic feedback schemes have also turned out to be attractive systems for chaos communication (Goedgebuer, Larger, and Porte, 1998; Abarbanel et al., 2001; Liu, Chen, and Tang, 2002), achieving bit rates up to $10 \mathrm{~Gb} / \mathrm{s}$ (Lavrov, Jacquot, and Larger, 2010).

In the schemes discussed so far, the information carriers are purely temporal signals. However, several schemes also exploit the spatial or spectral dimension to encode information. Optical devices exhibiting spatiotemporal or spectral-temporal chaos are the basis for constructing communication systems capable of transmitting information in space (or frequency) and time (White and Moloney, 1999; Garcia-Ojalvo and Roy, 2001).

An important advance in laser-based communication systems is the extension to novel schemes in which the information is exchanged simultaneously by two or more communication agents. Two bidirectionally delay-coupled semiconductor lasers cannot be employed for bidirectional communication due to spontaneous symmetry breaking (Heil, Fischer, Elsäßer, Mulet, and Mirasso, 2001). A possibility to overcome this limitation is to use extremely asymmetrical mutual injections, in a one-to-many and many-to-one communication scheme (Zhang et al., 2008). As discussed in Sec. III.D, a way to achieve stable identical synchronization between two delay-coupled oscillators is to implement common driving by a third chaotic laser. The feasibility of this approach in mutually coupled semiconductor lasers was explored via numerical simulations by Jiang et al. (2010), in which successful simultaneous message transmission was demonstrated with a bit rate higher than $10 \mathrm{~Gb} / \mathrm{s}$.

The security of most communication schemes presented so far in this section relies on the use of identical hardware with equal parameters of emitter and receiver. These parameters must be agreed on beforehand by the communication parties via a private channel. An eventual eavesdropper cannot decode an encrypted message as long as she or he does not have access to the private hardware and/or parameters. In contrast, there exists a separate family of communication schemes, which are still based on chaos synchronization, that can be used in the secure exchange of a private key through a public channel. This subset of communication schemes requires stable synchronization of two or more semiconductor lasers with symmetric information exchange. As mentioned in Sec. III.D, stable synchronization can be found in a scheme with two semiconductor lasers coupled via a relay laser (Fischer et al., 2006) or using self-feedback and mutual coupling (Klein, Gross, Rosenbluh et al., 2006; Vicente, Mirasso, and Fischer, 2007). In these schemes, all the parameters of the system can be of public knowledge and the private message can be recovered by a mutual chaos-pass filter principle (Klein, Gross, Rosenbluh et al., 2006). The working principle of the key exchange process is as follows: the two lasers synchronize only if the operating conditions (including the injection current) are the same in both lasers. However, if two different random bit streams modulate the injection current of each laser, the synchronization degree changes according to the bits encoded in each of the lasers. The modulation bit streams are generated locally in emitter and receiver and are, of course, different. The key exchange process is illustrated in Fig. 46, in which the injection currents of the lasers are simultaneously modulated with different pseudorandom bit sequences of small amplitude. When both parties of the communication send the same bit by modulating the bias current, the difference between the emission of the two lasers is zero. In that case, an eavesdropper cannot recognize whether a bit 0 or a bit 1 was sent. Thus, this type of mutually synchronized configuration can be used to 

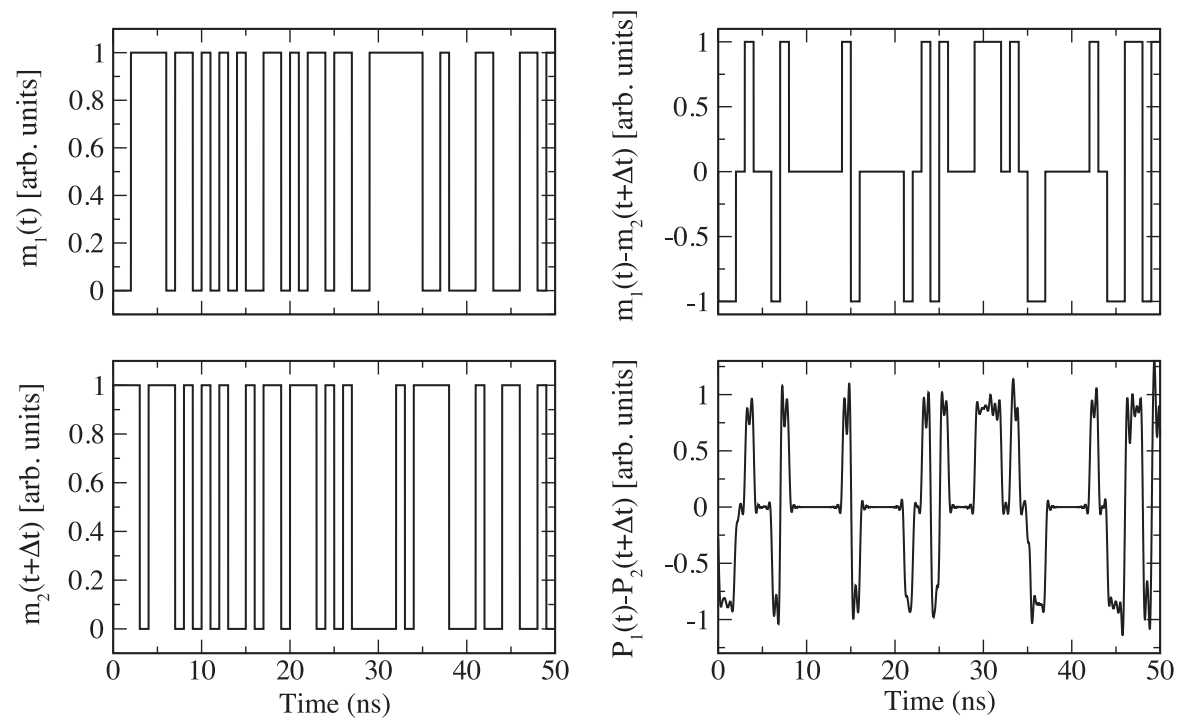

FIG. 46. Illustration of the key exchange process in a scheme with two mutually delay-coupled lasers mediated via a semitransparent mirror. $m_{1,2}$ are the bit sequences encoded by $\mathrm{SL}_{1,2}, m_{1}(t)-m_{2}(t+\Delta t)$ is the subtraction of the bit sequences with a time lag $\Delta t$. The synchronization error $P_{1}(t)-P_{2}(t+\Delta t)$, with $P_{1}-P_{2}$ being the output intensities of the corresponding delay-coupled lasers, provides information about the modulated sequences. The synchronization error was low-pass filtered to improve the signal-to-noise ratio. Details of the numerical simulations can be found in Vicente, Mirasso, and Fischer (2007).

simultaneously negotiate a key. The partners can agree to discard those bits that are different from each other while accepting those that coincide with each other. However, stability of the synchronization and bubbling effects need to be considered for practical implementations (Tiana-Alsina et al., 2012).

The synchronization process of two mutually delay-coupled deterministic chaotic maps was demonstrated both analytically and numerically by Kanter, Kopelowitz, and Kinzel (2008). The mutually transmitted signals were concealed by two commutative private filters, a convolution of the truncated time-delayed output signals or some powers of the delayed output signals. The task of a passive attacker was proven to be in the class of NP-complete problems, making this scheme attractive for public channel cryptography. Importantly, an eventual eavesdropper is not able to synchronize to any of the coupled lasers by tapping the communication channel, since the eavesdropper cannot engage into a bidirectional interaction with them without disturbing the synchronization.

\section{E. From lasers to the brain}

Laser and neuronal systems share some similar properties concerning the emergence of collective dynamics (Meucci et al., 2002) and synchronization phenomena (Fischer et al., 2006). In particular, propagation delays are common in both neural populations and coupled semiconductor lasers. Information transmission between brain regions typically takes significantly longer times (tens of ms) than the internal time scales of the neurons (of the order of $1 \mathrm{~ms}$ ). Initially, it was considered remarkable that two distant dynamical elements, being either neurons, neuron populations, or lasers, can synchronize at zero lag even in the presence of substantial delays in the information they exchange (Roelfsema et al., 1997; Fischer et al., 2006; Klein, Gross, Rosenbluh et al.,
2006; Vicente et al., 2008). These findings are important for applications in which the precise global timing is of relevance, since zero-lag collective synchronization can overcome the limitation of finite propagation delays.

As noted by Flunkert et al. (2010), zero-lag synchronization appears as a consequence of the network topology. The relay topology that inherently allows for zero-lag synchronization is a candidate to explain the phenomenology of long-range synchronization observed in the brain. Zero-lag synchronization was observed across several species with different brain sizes and at different stages of the developmental growth of brain structures. It is discussed as an important mechanism for coherent perception (Singer, 1999). As introduced by Vicente et al. (2008), two distant populations of neurons can exhibit zero-lag synchrony via dynamical relaying in a wide range of propagation delays. Figure 47 shows the results obtained when two large neuron populations are connected via a relaying population. In this case, the outer populations 1 and 3 are synchronized at zero lag [see Fig. 47(e)], while the middle population is synchronized with a time shift. Simple models considering the thalamus (Gollo, Mirasso, and Villa, 2010) or the hippocampus (Gollo et al., 2011) as relay elements have shown that zerolag long-range synchronization can be achieved under certain conditions.

The existence of zero-lag synchronization between distant neuron populations can also be understood in terms of the directed network loops in the coupling topology. The number of synchronized clusters of neuron activity matches the GCD of all existing network loops (Kanter, Kopelowitz et al., 2011). The GCD principle is illustrated in Fig. 48 (left column), in which different network topologies yield different numbers of synchronized clusters according to the different number of network loops in each configuration. Clusters with synchronized activity are encoded with a common color. 

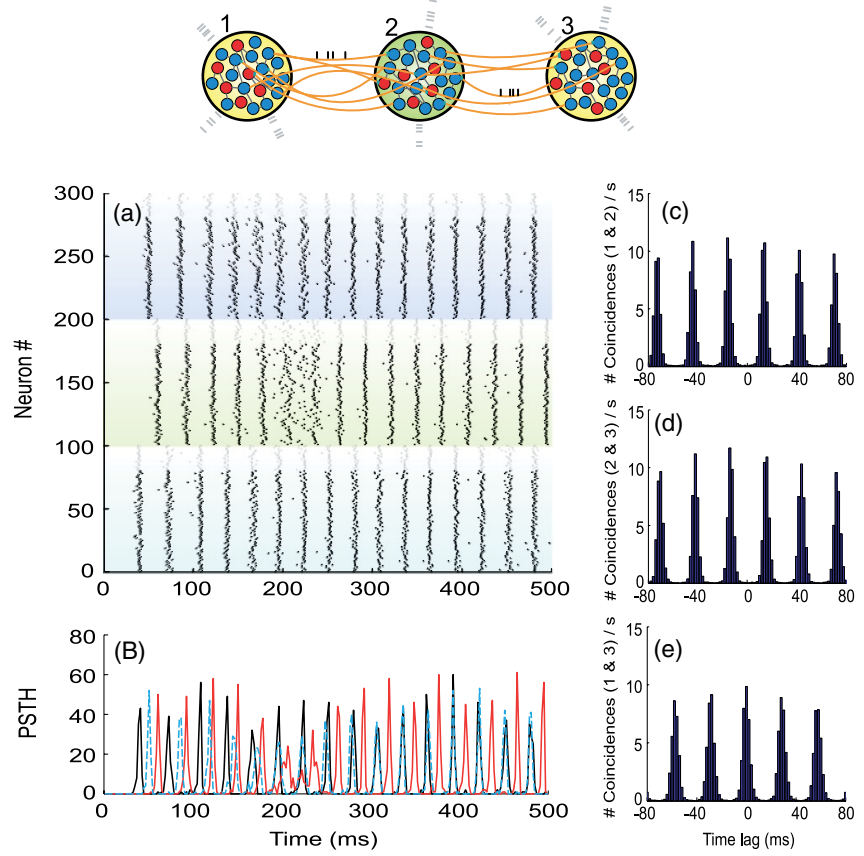

FIG. 47 (color online). Three neuron populations (top panel) interacting through dynamical relaying. (a) Raster plots of a selection of 300 neurons from each population, 1-3 from bottom to top. (b) Firing histogram of 100 neurons from each population; black, solid gray, and dashed gray for populations 1,2, and 3, respectively. (c) Cross correlogram between neurons of populations 1 and 2; (d) populations 2 and 3; and (e) populations 1 and 3. Adapted from Vicente et al., 2008.

The synchronization of neural activity has been confirmed by numerical simulations and controlled in vitro experiments (Kanter, Kopelowitz et al., 2011; Vardi et al., 2012). Figure 48 (right column) presents the appearance of synchronized clusters in different neuronal circuits. The temporal patterns of reverberating synchronized neural electrical activity are generated by sequential stimulation of a circuit of neurons embedded within a large scale of in vitro cortical cells (Vardi et al., 2012).

For neuronal systems, delay, diversity, and the role of topology play an important role. For example, information processing in the brain takes a certain time that depends, among other characteristics, on the modality of the sensory input. Visual stimuli take tens to hundreds of milliseconds to reach the visual cortex, while auditory stimuli take 1 to $10 \mathrm{~ms}$ to reach the auditory cortex. Besides the cortex itself, circuits involving cortical areas and subcortical structures are of major relevance and it is essential to understand their behavior and the effects on possible failures. The thalamus (the main relay element in the brain), the hippocampus, and other subcortical structures play a crucial role in communicating and distributing information to and from the cortex. Many pathologies such as epilepsy, Alzheimer's, or schizophrenia are due to an excess or loss of activity in these networks, often related to synchronization. The search for simple circuits that account for such states in terms of alteration of the coupling strengths, coupling delays, and changes in the dynamics of the neurons could contribute to shedding light onto such challenging issues. (a)<smiles>C[Al](C)O[Al]</smiles>

(b)

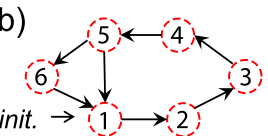

(c)

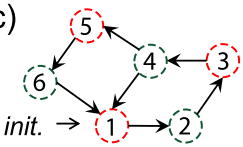

(d)

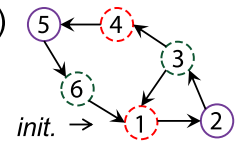

(e)
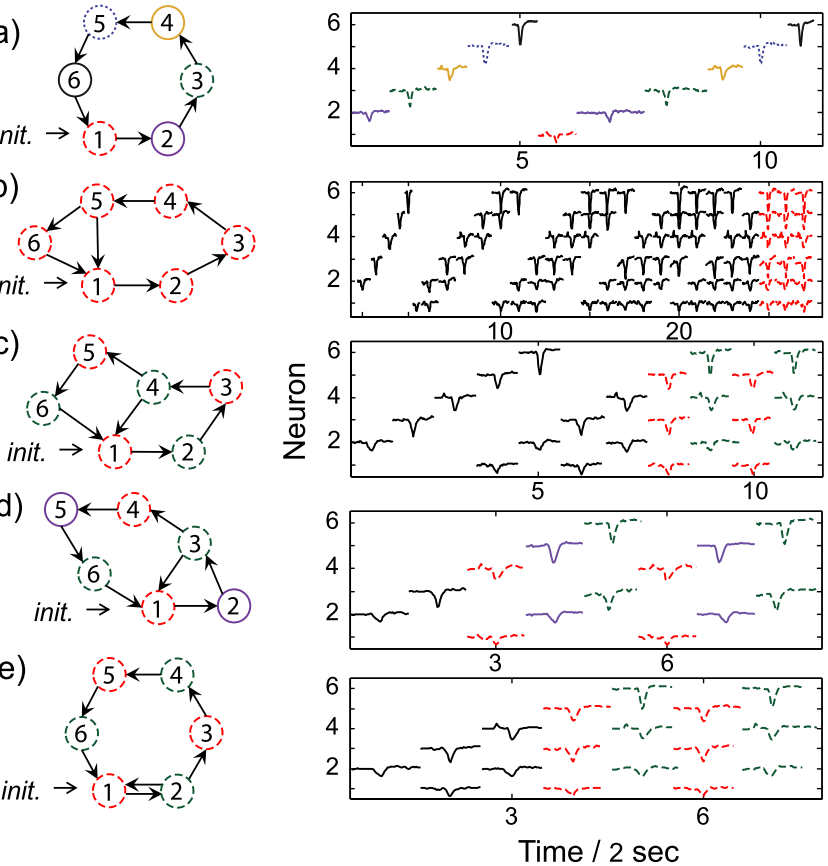

FIG. 48 (color online). Transients and reverberating activity patterns in six neuron circuits, using a conditioned stimulation protocol following the circuit connectivity (arrows in the left column). The right column shows spike trains of the six recording electrodes. (a) A directed loop with six activity groups of one neuron each. (b) $\operatorname{GCD}(5,6)=1$ group of reverberating activity. (c) $\operatorname{GCD}(4,6)=2$ groups of reverberating activity. (d) $\operatorname{GCD}(3,6)=3$ groups of reverberating activity. (e) A single directed loop with one bidirectional coupling forming a loop of size $2, \operatorname{GCD}(2,6)=2$ groups of reverberating activity as in (c). Adapted from Vardi et al., 2012.

Neuronal circuits involving the thalamus as a relay element are essential, since virtually all information reaching the cortex must first pass through the thalamus. In Fig. 49 we show as an example a first order thalamic relay (left) that represents the first relay of subcortical information of a particular type to a primary cortical area. A higher order relay (right) relays information from layer 5 of one cortical area to another cortical area. Similar kinds of circuits can be implemented in fiber-based semiconductor laser setups. The possibility of injecting information via optical or electrical inputs into the lasers could mimic different sensory modalities. The freedom to couple different elements in different configurations and to choose their coupling strengths and delays will allow one to analyze a large variety of regimes and to characterize different possible emergent behaviors and failures. Even simple circuits based on delay-coupled semiconductor lasers could contribute to the understanding of the dynamical properties of neuronal networks.

The similarities between the dynamical properties of coupled neural populations and simpler delay-coupled nonlinear dynamical elements have also inspired the development of a novel information processing concept. It was shown that a single nonlinear element with delay is capable of performing complex classification tasks by using its transient response when its own dynamics are mixed with an external information stream (Appeltant et al., 2011). The 


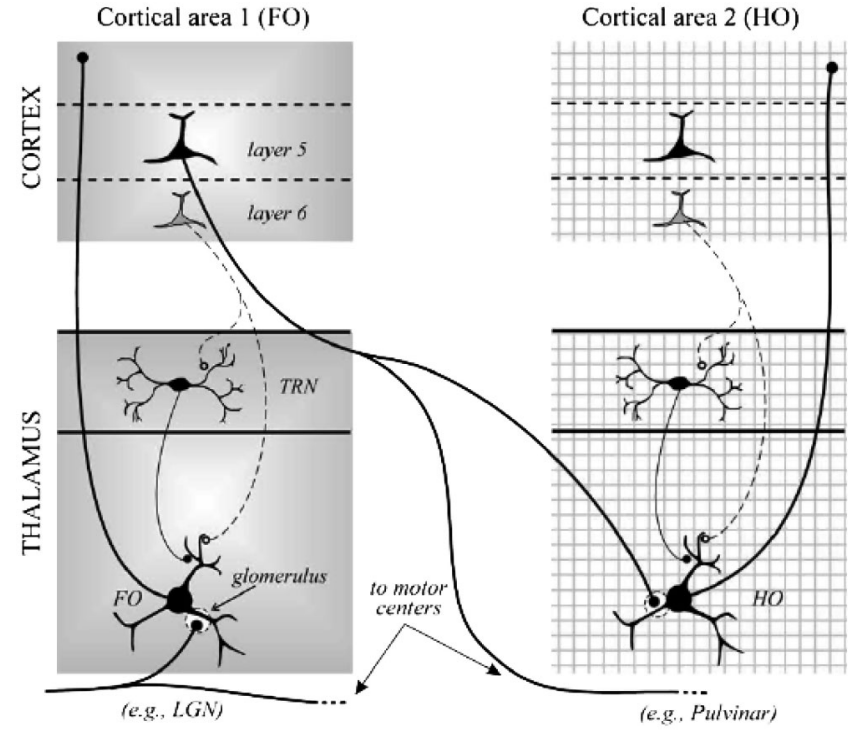

FIG. 49. Role of higher order thalamic relays in corticocortical communication. The suggested route of much of this communication involves a projection from layer 5 of the cortex to a higher order thalamic relay to another cortical area. In question is the function, driver or modulator, of the direct corticocortical projections. From Sherman, 2005.

implementation of this information processing concept was recently extended to photonics by using an optoelectronic oscillator (Larger et al., 2012; Paquot et al., 2012). As noted by Larger et al. (2012), the concept is rather independent of the precise nonlinear element used, even if the overall performance of the system depends significantly on the operating conditions. The first successful extensions of this paradigm to all-optical information processing applications using semiconductor lasers or semiconductor optical amplifiers with delayed feedback have been reported (Duport et al., 2012; Brunner, Soriano et al., 2013). Bioinspired photonic information processing is further discussed in Sec. VI.B.

\section{CONCLUSIONS AND FUTURE PERSPECTIVES}

\section{A. Conclusions}

As detailed previously, semiconductor lasers with delayed coupling have proven to be versatile and well-controlled dynamical systems. Meanwhile they have gained the maturity to be employed in applications. Several applications have already been implemented, based on delayed-feedback lasers or small motifs of coupled lasers, harnessing their complex dynamics. These applications include encrypted communication with chaotic carriers, classical key exchange, rainbow refractometry, sensing, and fast random bit sequence generation. Nevertheless, their full capabilities will, in our opinion, only be exploited in the future. The fundamental insights gained in recent years on delay-coupled network configurations, the availability of new mathematical tools to tackle delay systems, in combination with novel devices and technologies that have been developed, open research perspectives, from which further applications are emerging. One promising area is related to unconventional and brain-inspired computational techniques. Delay-coupled semiconductor laser systems are promising candidates to implement bioinspired information processing concepts. This perspective will be elaborated on in Sec. VI.B. From a technological viewpoint, recent advances in active-passive integration and realization of PIC open perspectives for integration of these concepts and might even lead to scalability. These perspectives will be discussed in Sec. VI.C. Finally, we will comment on possible cross fertilizations between the studies of networks of delay-coupled lasers with other areas of science.

\section{B. Bioinspired photonic information processing}

Only now are complex behavior, the influence of delayed coupling, and its functional role being recognized and addressed in the brain, in social interactions, and in dynamical systems in general. A key objective is the better understanding of how in such systems information is encoded, integrated, propagated, processed, and stored. From a fundamental point of view, Crutchfield, Ditto, and Sinha (2010) emphasized that every dynamical process represents an intrinsic processing of information. The difficulty is to employ dynamical systems for a certain task and to extract the processed information efficiently. But more and more, standard methods of computation are being challenged by alternative, unconventional computational paradigms. The hope related to these approaches, besides the fundamental understanding, is for faster and more energy efficient computing, with not too demanding hardware.

Many different concepts have been introduced to mimic information processing in the brain, including neural network methods, support vector machines, and reservoir computing. They can indeed perform certain tasks efficiently that are usually deemed computationally hard.

Reservoir computing (Jaeger and Haas, 2004; Buonomano and Maass, 2009) is a recently introduced machine-learning paradigm that exhibits state-of-the-art performance for processing empirical data. The main inspiration underlying reservoir computing is the insight that neural systems can process information by generating patterns of transient activity which are excited by input sensory signals (Rabinovich, Huerta, and Laurent, 2008). It is based on a reservoir, usually comprising a large complex recurrent network and an input and output layer. The input layer connects different input nodes randomly to the nodes of the reservoir. The connectivity in the reservoir is left unchanged and only the connection weights to the output layer are trained. Computationally hard tasks, such as chaotic time series prediction (Jaeger and Haas, 2004) or speech recognition (Verstraeten et al., 2005), can be addressed, yielding high performance.

Many of these unconventional computation methods require a complex network and its emerging complex response. Traditionally, recurrent networks of pulse-coupled spiking elements such as neurons have been used. Computations emerge from the properties of the individual coupled elements and the inherent network dynamics. In most cases, this complex network is simulated on a computer, therefore having to be serialized again. Hardware implementations of complex networks as computational systems are rather scarce. 
The particular features of delay systems enable even a single delayed-feedback system to replace a complex network and perform brain-inspired analog computing. An analog computation concept based on a delayed-feedback system has been shown to perform certain tasks, such as spoken digit recognition and time series prediction, as well as other types of machine-learning approaches or even outperforming them (Appeltant et al., 2011; Duport et al., 2012; Larger et al., 2012; Paquot et al., 2012; Brunner, Soriano et al., 2013). Utilizing delay, the approach benefits from a radical simplification of the required hardware from hundreds or thousands of nodes to one or very few analog hardware nodes. Therefore, analog computation using delay-coupled systems has the major advantage of a possible implementation in photonics hardware. Photonics provides some advantages, although a number of challenges needs to be faced. Because of the delayed feedback, the dynamical response of the system is determined by both the input drive and the past activity of the processor. Thus, via the delayed-feedback loop the past activity is being introduced into the system. The central computational concept is then based on the mapping of a dynamical input state onto a high-dimensional state space. For this, the input streams are converted into spatiotemporally distributed states in the feedback line. Specific computations can then be realized by a classification of these spatiotemporally distributed states into output classes based on a trained linear readout. Therefore, these analog computations based on dynamical systems are composed of two steps: first the nonlinear transformation of the input via the processor activity that serves as a dimensionality expansion, and second a classification of the processor states that makes the computation meaningful and problem oriented.

With motifs or small networks of delay-coupled lasers, a compromise between computational speed and flexibility on the one side and hardware efficiency on the other side might be found.

\section{Toward integrated complex photonics}

From a technological viewpoint, recent advances in active-passive integration and realization of PIC provide perspectives for integrated complex photonic devices, which implement the concepts discussed in this review and promise scalability.

\section{Integrated lasers with delayed feedback}

In Sec. II.D.2, we discussed some integrated laser structures with delayed feedback. Ushakov et al. (2004) introduced a device with delayed feedback, originating from an integrated passive section of $200 \mu \mathrm{m}$ length, exhibiting delayed-feedback-induced self-pulsations. In addition, even amplified feedback schemes have been implemented (Bauer et al., 2004), for which phase and strength of the feedback can be separately tuned. Such lasers showed different kinds of self-pulsations and chaotic behavior. Even monolithic integrated laser structures based on delayed feedback that were particularly designed to exhibit chaotic emission have been realized (Argyris et al., 2008). They consist of a distributed feedback laser, a passive resonator, and active elements that control the optical feedback properties. A different kind of
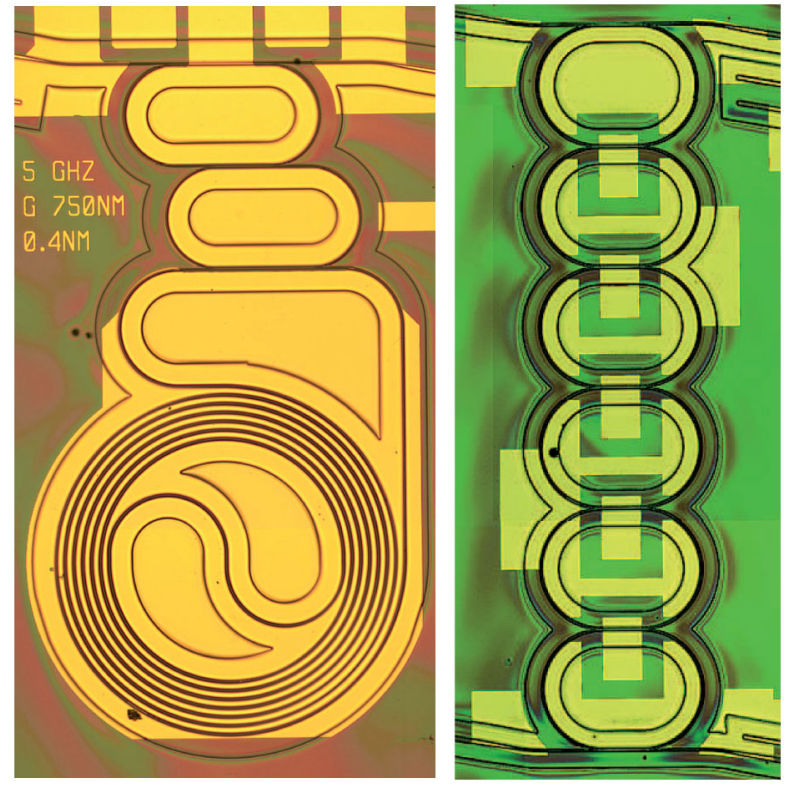

FIG. 50 (color online). Left panel: Chip of semiconductor ring lasers, emitting at $\lambda=1.55 \mu \mathrm{m}$. The top ring is connected to a bus waveguide and the bottom ring to a spiral, implementing delayed feedback. Right panel: Chip of six coupled ring lasers (cavity length $1250 \mu \mathrm{m}$ ), illustrating the integration perspectives for coupled lasers. Courtesy of Marc Sorel, University of Glasgow.

integrated chaotic emitter was reported by Yousefi et al. (2007), based on a colliding-pulse mode-locked structure. Originally built for optical short-pulse generation, they concluded that, based on their observations, integrated active photonic devices intrinsically exhibit nonlinear dynamics.

Sunada et al. (2011) recently reported on a novel compact chaos laser device design. In order to achieve strong feedback for broadband chaos generation, the structure consists of a ring-type delayed optical feedback configuration using a passive ring waveguide monolithically integrated with a single-mode DFB laser, two semiconductor optical amplifiers, and a fast photodiode. Optical feedback strengths of about $10 \%$ are possible, resulting in broadband chaotic signals with spectra that are up to $10 \mathrm{GHz}$. Such structures further illustrate the possibilities of realizing compact and robust devices for complex photonics. They provide a combination of performance, small size, and low cost, opening perspectives for applications in sensing, communication, and signal processing applications.

How significant delays can be integrated on a chip is illustrated in the left panel of Fig. 50, depicting integrated ring lasers, one of which is coupled to a long delay via a spiral-shaped waveguide.

The above-mentioned structures illustrate the advances in active-passive integration. Such techniques offer further perspectives for the realization of integrated delay-coupled laser structures with different functional elements.

\section{Integrated coupled lasers and photonic integrated circuits}

Delay-coupled laser structures have already been implemented as well. Wünsche et al. (2005) reported on an integrated tandem device in which two DFB lasers are arranged on a single chip separated by a $300 \mu \mathrm{m}$ long passive 
waveguide section. Despite the short coupling path, this laser shows typical delay-induced phenomena. Further photonic integrated circuits comprising coupled laser devices have already been implemented, e.g., to enhance the relaxation oscillation frequency (Tauke-Pedretti et al., 2011). In addition to approaches using linear cavity configurations, integrated ring lasers have recently gained much attention. They might allow for larger scale integrated coupled laser structures. The right panel in Fig. 50 shows a chip of six evanescently coupled semiconductor ring lasers, illustrating the integration capabilities.

An important general trend in photonics technology is to move toward large-scale photonic integration of components with complex functionalities. Networks of monolithically coupled devices have come into reach. Such photonic integrated devices allow one to realize and control complex dynamical behavior in a reliable manner, which can then be exploited in various applications.

Besides integrated structures, intermediate solutions might be adopted, based on arrays of lasers that can be coupled via an external-cavity configuration.

\section{Final remarks}

In addition to the photonics-oriented aspects, it is worth mentioning that networks of delay-coupled elements are of relevance in other areas as well. As interactions in coupled systems are mediated by signals which travel at finite speed, many complex dynamical systems are subjected to time delay. Laser systems, with their well-controlled parameter conditions, might again serve as test-bed systems in order to study phenomena such as the emergence of collective behavior, the role of topology, the role of diversity, robustness of networks against various types of perturbations, the appearance of critical events, or the identification of their precursors. The results obtained with these models would have relevance in areas as diverse as neuroscience, critical infrastructure, communication networks, and even in medicine and economics.

Altogether, the availability of high-quality telecommunication components, advances in technology, and the cross fertilization of photonics with other fields of science in which delay-coupled networks play a role, offer qualitatively new chances. Novel applications have already been implemented, so far appearing rather as isolated solutions to particular problems. With these new perspectives, a whole infrastructure of applications can be developed that might contribute to solving major issues in today's communication and IT systems, including privacy, computational efficiency, or power consumption. Ultimately, a new paradigm of functional complex photonics could emerge.

\section{ACKNOWLEDGMENTS}

We acknowledge valuable discussions with and helpful comments from many colleagues, including Daniel Brunner, Pere Colet, Valentin Flunkert, Konstantin Hicke, Thomas Jüngling, Wolfgang Kinzel, Cristina Masoller, Valerie Moliere, Neus Oliver, Xavier Porte, Eckehard Schöll, and Carme Torrent. We particularly thank Laurent Larger for the careful reading of the manuscript and for providing many valuable comments. Furthermore, we acknowledge the following organizations and projects: Ministerio de Ciencia e Innovacion, Comunitat Autònoma de les Illes Balears and FEDER (Spain) under Contracts No. TEC2009-14101 (DeCoDicA), No. TEC2012-36335 (TRIPHOP), No. FIS2009-13360, No. FIS2007-60327 (FISICOS), and No. FIS2012-30634 (Intense@cosyp), Grups Competitius, the European Commission for EC FP7 Project PHOCUS under Grant No. 240763, and Institució Catalana de Recerca i Estudis Avançats (ICREA) under its Academia program.

\section{REFERENCES}

Abarbanel, H. D. I., M. B. Kennel, L. Illing, S. Tang, H. F. Chen, and J.M. Liu, 2001, "Synchronization and communication using semiconductor lasers with optoelectronic feedback," IEEE J. Quantum Electron. 37, 1301.

Abarbanel, H.D.I., N.F. Rulkov, and M. M. Sushchik, 1996, "Generalized synchronization of chaos: the auxiliary system approach," Phys. Rev. E 53, 4528.

Agrawal, Govind P., 2010, Fiber-Optic Communication Systems, edited by Kai Chang, Wiley Series in Microwave and Optical Engineering (Wiley, New York), 4th ed.

Ahlers, V., U. Parlitz, and W. Lauterborn, 1998, "Hyperchaotic dynamics and synchronization of external-cavity semiconductor lasers," Phys. Rev. E 58, 7208.

Albert, Ferdinand, Caspar Hopfmann, Stephan Reitzenstein, Christian Schneider, Sven Höfling, Lukas Worschech, Martin Kamp, Wolfgang Kinzel, Alfred Forchel, and Ido Kanter, 2011, "Observing chaos for quantum-dot microlasers with external feedback," Nat. Commun. 2, article no. 366.

Albert, J., M. C. Soriano, I. Veretennicoff, K. Panajotov, J. Danckaert, P. A. Porta, D. P. Curtin, and J. G. McInerney, 2004, "Laser Doppler velocimetry with polarization-bistable VCSELs," IEEE J. Quantum Electron. 10, 1006.

Albert, Réka, and Albert-László Barabási, 2002, "Statistical mechanics of complex networks," Rev. Mod. Phys. 74, 47.

Alferov, Zhores I., 2001, "Nobel Lecture : The double heterostructure concept and its applications in physics, electronics, and technology," Rev. Mod. Phys. 73, 767.

Amann, A., A. Pokrovskiy, S. Osborne, and S. O'Brien, 2008, "Complex Networks based on Discrete-mode Lasers," J. Phys. Conf. Ser. 138, 012001.

Andersen, O. K., A. P. A. Fischer, I. C. Lane, E. Louvergneaux, S. Stolte, and D. Lenstra, 1999, "Experimental stability diagram of a diode laser subject to weak phase-conjugate feedback from a rubidium vapor cell,' IEEE J. Quantum Electron. 35, 577.

Annovazzi-Lodi, V., S. Donati, and A. Sciré, 1996, "Synchronization of chaotic injected-laser systems and its application to optical cryptography," IEEE J. Quantum Electron. 32, 953.

Appeltant, L., M. C. Soriano, G. Van der Sande, J. Danckaert, S. Massar, J. Dambre, B. Schrauwen, C. R. Mirasso, and I. Fischer, 2011, "Information processing using a single dynamical node as complex system," Nat. Commun. 2, 468.

Argyris, A., S. Deligiannidis, E. Pikasis, A. Bogris, and D. Syvridis, 2010, "Implementation of $140 \mathrm{~Gb} / \mathrm{s}$ true random bit generator based on a chaotic photonic integrated circuit," Opt. Express 18, 18763.

Argyris, A., M. Hamacher, K. Chlouverakis, A. Bogris, and D. Syvridis, 2008, "Photonic Integrated Device for Chaos Applications in Communications," Phys. Rev. Lett. 100, 194101. 
Argyris, Apostolos, Evangellos Grivas, Michael Hamacher, Adonis Bogris, and Dimitris Syvridis, 2010, "Chaos-on-a-chip secures data transmission in optical fiber links." Opt. Express 18, 5188.

Argyris, Apostolos, Dimitris Syvridis, Laurent Larger, V. Annovazzi-Lodi, Pere Colet, Ingo Fischer, J. Garcia-Ojalvo, C.R. Mirasso, Luis Pesquera, and K. A. Shore, 2005, "Chaosbased communications at high bit rates using commercial fibreoptic links," Nature (London) 438, 343.

Aviad, Y., I. Reidler, M. Zigzag, M. Rosenbluh, and I. Kanter, 2012, "Synchronization in small networks of time-delay coupled chaotic diode lasers," Opt. Express 20, 4352.

Azouigui, S., et al., 2007, "Coherence collapse and low-frequency fluctuations in quantum-dash based lasers emitting at $1.57 \mu \mathrm{m}$," Opt. Express 15, 14155.

Bachmann, F., P. Loosen, and R. Poprawe, 2010, Eds., High Power Diode Lasers: Technology and Applications, Springer Series in Optical Sciences (Springer, New York).

Balachandran, B., T. Kamr-Nagy, and D. Gilsinn, 2009, Delay Differential Equations, Recent Advances and New Directions (Springer, New York).

Balle, Salvador, 1998, "Simple analytical approximations for the gain and refractive index spectra in quantum-well lasers," Phys. Rev. A 57, 1304.

Barchanski, A., T. Gensty, C. Degen, I. Fischer, and W. Elsässer, 2003, "Picosecond emission dynamics of vertical-cavity surfaceemitting lasers: Spatial, spectral, and polarization-resolved characterization," IEEE J. Quantum Electron. 39, 850.

Bauer, S., O. Brox, J. Kreissl, B. Sartorius, M. Radziunas, J. Sieber, H.-J. Wünsche, and F. Henneberger, 2004, "Nonlinear dynamics of semiconductor lasers with active optical feedback," Phys. Rev. E 69, 163901.

Bellman, R. E., and J. M. Danskin, 1954, A Survey of the Mathematical Theory of Time Lag, Retarded Control, and Hereditary Processes, Rand Corporation Technical Report No. R-256.

Boccaletti, S., J. Kurths, G. Osipov, D. L. Valladares, and C.S. Zhou, 2002, "The synchronization of chaotic systems," Phys. Rep. 366, 1.

Boccaletti, S., V. Latora, Y. Moreno, M. Chavez, and D.-U. Hwang, 2006, "Complex networks: Structure and dynamics," Phys. Rep. 424, 175.

Bogatov, A. P., P. G. Eliseev, L. P. Ivanov, A. S. Logginov, A. Manko, and K. Ya. Senatorov, 1973, "Study of the Single-Mode Injection Laser," IEEE J. Quantum Electron. 9, 392.

Botez, D., and D.E. Ackey, 1986, "Phase-locked arrays of semiconductor diode-lasers," IEEE Circuits Devices Mag. 2, 8.

Botez, D., and L.J. Mawst, 1996, "Phase-locked laser arrays revisited," IEEE Circuits Devices Mag. 12, 25.

Broom, R., E. Mohn, and C. Risch, 1970, "Microwave selfmodulation of a diode laser coupled to an external cavity," IEEE J. Quantum Electron. 6, 328.

Broom, R. F., 1969, "Self modulation at gigahertz frequencies of a diode laser coupled to an external cavity," Electron. Lett. 5, 571.

Brunner, Daniel, Miguel C. Soriano, Claudio R. Mirasso, and Ingo Fischer, 2013, "Parallel photonic information processing at gigabyte per second data rates using transient states," Nat. Commun. 4, 1364.

Brunner, Daniel, Xavier Porte, Miguel C. Soriano, and Ingo Fischer, 2012, "Real-time frequency dynamics and high-resolution spectra of a semiconductor laser with delayed feedback," Sci. Rep. 2, 732.

Buldu, J. M., Raul Vicente, Toni Perez, Claudio R. Mirasso, M. C. Torrent, and J. Garcia-Ojalvo, 2002, "Periodic entrainment of power dropouts in mutually coupled semiconductor lasers," Appl. Phys. Lett. 81, 5105.

Buldú, J., J. García-Ojalvo, Claudio Mirasso, M. Torrent, and J. Sancho, 2001, "Effect of external noise correlation in optical coherence resonance,” Phys. Rev. E 64, 051109.
Buldú, J. M., D. R. Chialvo C.R. Mirasso M.C. Torrent, and J. García-Ojalvo, 2003, "Ghost resonance in a semiconductor laser with optical feedback," Europhys. Lett. 64, 178.

Buldú, J. M., J. García-Ojalvo, C.R. Mirasso, and M. C. Torrent, 2002, "Stochastic entrainment of optical power dropouts," Phys. Rev. E 66, 021106.

Buldú, J. M., J. García-Ojalvo, and M. C. Torrent, 2004, "Delayinduced resonances in an optical system with feedback," Phys. Rev. E 69, 046207.

Buldú, J.M., M.C. Torrent, and J. Garcia-Ojalvo, 2007, "Synchronization in Semiconductor Laser Rings," J. Lightwave Technol. 25, 1549.

Buldú, Javier. M., C. M. González, J. Trull, M. C. Torrent, and J. García-Ojalvo, 2005, "Coupling-mediated ghost resonance in mutually injected lasers," Chaos 15, 013103.

Bungay, Sharene D., and Sue Ann Campbell, 2007, "Patterns of Oscillation in a Ring of Identical Cells with Delayed Coupling," Int. J. Bifurcation Chaos Appl. Sci. Eng. 17, 3109.

Bünner, M. J., M. Ciofini, A. Giaquinta, R. Hegger, H. Kantz, R. Meucci, and A. Politi, 2000, "Reconstruction of systems with delayed feedback: II. Application," Eur. Phys. J. D 10, 177.

Buonomano, Dean. V., and Wolfgang Maass, 2009, "Statedependent computations: spatiotemporal processing in cortical networks," Nat. Rev. Neurosci. 10, 113.

Buric, N., and D. Todorovic, 2003, "Synchronization of hyperchaotic systems with delayed bidirectional coupling," Phys. Rev. E 68, 066218.

Carroll, Olwen, Ian O'Driscoll, Stephen P. Hegarty, Guillaume Huyet, John Houlihan, Evgeny Viktorov, and Paul Mandel, 2006, "Feedback induced instabilities in a quantum dot semiconductor laser," Opt. Express 14, 10831.

Chi, Mingjun, Birgitte Thestrup, and Paul Michael Petersen, 2005, "Self-injection locking of an extraordinarily wide broad-area diode laser with a 1000-microm-wide emitter," Opt. Lett. 30, 1147.

Chiang, M.C., H.F. Chen, and J.M. Liu, 2005, "Experimental synchronization of mutually coupled semiconductor lasers with optoelectronic feedback," IEEE J. Quantum Electron. 41, 1333.

Choe, Chol-Ung, Thomas Dahms, Philipp Hövel, and Eckehard Schöll, 2010, "Controlling synchrony by delay coupling in networks: From in-phase to splay and cluster states," Phys. Rev. E 81, 025205.

Chow, W., M. Sargent, and S. Koch, 1997, Semiconductor Laser Physics (Springer, Berlin/Heidelberg).

Cohen, E., M. Rosenbluh, and I. Kanter, 2012, "Phase transition in crowd synchrony of delay-coupled multilayer laser networks," arXiv:1204.3712.

Cohen, Seth. D., Hugo L.D. de S. Cavalcante, and Daniel J. Gauthier, 2011, "Subwavelength position sensing using nonlinear feedback and wave chaos," Phys. Rev. Lett. 107, 254103.

Colet, P., and R. Roy, 1994, "Digital communication with synchronized chaotic lasers," Opt. Lett. 19, 2056.

Crutchfield, James. P., William L. Ditto, and Sudeshna Sinha, 2010, "Introduction to focus issue: intrinsic and designed computation: information processing in dynamical systems-beyond the digital hegemony," Chaos 20, 037101.

Cuomo, K. M, A. V. Oppenheim, and S.H. Strogatz, 1993, "Synchronization of Lorenz-based chaotic circuits with applications to communications," IEEE Trans. Circuits Syst. II 40, 626.

Dahms, T., P. Hövel, and E. Schöll, 2008, "Stabilizing continuouswave output in semiconductor lasers by time-delayed feedback," Phys. Rev. E 78, 056213.

Dahms, T., J. Lehnert, and E. Schöll, 2012, "Cluster and group synchronization in delay-coupled networks," arXiv:1203.4916. 
DeFreez, R. K., D. J. Bossert, N. Yu, K. Hartnett, R. A. Elliott, and H. G. Winful, 1988, "Spectral and picosecond temporal properties of flared guide $Y$-coupled phase-locked laser arrays," Appl. Phys. Lett. 53, 2380.

Deng, T., G.-Q. Xia, Z.-M. Wu, X.-D. Lin, and J.-G. Wu, 2011, "Chaos synchronization in mutually coupled semiconductor lasers with asymmetrical bias currents," Opt. Express 19, 8762.

Detoma, E, B. Tromborg, and I. Montrosset, 2005, "The complex way to laser diode spectra: example of an external cavity laser strong optical feedback," IEEE J. Quantum Electron. 41, 171.

Dhamala, Mukeshwar, Viktor Jirsa, and Mingzhou Ding, 2004, "Enhancement of Neural Synchrony by Time Delay," Phys. Rev. Lett. 92, 074101.

D’Huys, O., I. Fischer, J. Danckaert, and R. Vicente, 2011, "Role of delay for the symmetry in the dynamics of networks," Phys. Rev. E 83, 046223.

D'Huys, O., R. Vicente, J. Danckaert, and I. Fischer, 2010, "Amplitude and phase effects on the synchronization of delaycoupled oscillators," Chaos 20, 043127.

D'Huys, O., R. Vicente, T. Erneux, J. Danckaert, and I. Fischer, 2008, "Synchronization properties of network motifs: influence of coupling delay and symmetry," Chaos 18, 037116.

Dodla, Ramana, Abhijit Sen, and George Johnston, 2004, "Phaselocked patterns and amplitude death in a ring of delay-coupled limit cycle oscillators," Phys. Rev. E 69, 056217.

Donati, S., 2004, Electro-optical Instrumentation: Sensing and Measuring with Lasers (Prentice-Hall, Englewood Cliffs, NJ).

Donati, S., G. Giuliani, and S. Merlo, 1995, "Laser diode feedback interferometer for measurement of displacements without ambiguity," IEEE J. Quantum Electron. 31, 113.

Donati, S., and C.R. Mirasso, 2002, "Introduction to the feature section on optical chaos and applications to cryptography," IEEE J. Quantum Electron. 38, 1138.

Duport, François, Bendix Schneider, Anteo Smerieri, Marc Haelterman, and Serge Massar, 2012, "All-optical reservoir computing," Opt. Express 20, 22783.

Elowitz, Michael. B., and Stanislas Leibler, 2000, "A synthetic oscillatory network of transcriptional regulators," Nature (London) 403, 335.

Engelborghs, K., T. Luzyanina, and D. Roose, 2002, “Numerical bifurcation analysis of delay differential equations using ddebiftool," ACM Trans. Math. Softw. 28, 1.

Englert, A., S. Heiligenthal, W. Kinzel, and I. Kanter, 2011, "Synchronization of chaotic networks with time-delayed couplings: An analytic study," Phys. Rev. E 83, 046222.

Englert, A., W. Kinzel, Y. Aviad, M. Butkovski, I. Reidler, M. Zigzag, I. Kanter, and M. Rosenbluh, 2010, "Zero lag synchronization of chaotic systems with time delayed couplings," Phys. Rev. Lett. 104, 114102.

Erneux, Thomas, 2009, Applied Delay Differential Equations, Surveys and Tutorials in the Applied Mathematical Sciences (Springer, New York).

Erneux, Thomas, and Pierre Glorieux, 2010, Laser Dynamics (Cambridge University Press, Cambridge, UK).

Erzgräber, H., B. Krauskopf, and D. Lenstra, 2005, “Mode structure of delay-coupled semiconductor lasers: influence of the pump current," J. Opt. B 7, 361.

Erzgräber, H., B. Krauskopf, D. Lenstra, A. Fischer, and G. Vemuri, 2006, "Frequency versus relaxation oscillations in a semiconductor laser with coherent filtered optical feedback," Phys. Rev. E 73, 055201.

Erzgräber, H., D. Lenstra, B. Krauskopf, E. Wille, M. Peil, I. Fischer, and W. Elsäßer, 2005, "Mutually delay-coupled semiconductor lasers: Mode bifurcation scenarios," Opt. Commun. 255, 286.
Fabiny, L., P. Colet, R. Roy, and D. Lenstra, 1993, "Coherence and phase dynamics of spatially coupled solid-state lasers," Phys. Rev. A 47, 4287.

Feinberg, Jack, 1982, "Self-pumped, continuous-wave phase conjugator using internal reflection," Opt. Lett. 7, 486.

Ferguson, N., B. Schneier, and T. Kohno, 2010, Cryptography Engineering: Design Principles and Practical Applications (Wiley Publishing, New York).

Fischer, Alexis, Mirvais Yousefi, Daan Lenstra, Michael Carter, and Gautam Vemuri, 2004, "Filtered Optical Feedback Induced Frequency Dynamics in Semiconductor Lasers," Phys. Rev. Lett. 92, 023901.

Fischer, I., O. Hess, W. Elsäßer, and E. Göbel, 1996, “Complex spatio-temporal dynamics in the near-field of a broad-area semiconductor laser," Europhys. Lett. 35, 579.

Fischer, I., G. H. van Tartwijk, A. M. Levine, W. Elsäßer, E. Göbel, and D. Lenstra, 1996, "Fast pulsing and chaotic itinerancy with a drift in the coherence collapse of semiconductor lasers," Phys. Rev. Lett. 76, 220.

Fischer, Ingo, Yun Liu, and Peter Davis, 2000, "Synchronization of chaotic semiconductor laser dynamics on subnanosecond time scales and its potential for chaos communication," Phys. Rev. A 62, 011801(R).

Fischer, Ingo, Raúl Vicente, Javier M. Buldú, Michael Peil, Claudio R. Mirasso, M. C. Torrent, and Jordi García-Ojalvo, 2006, "Zerolag long-range synchronization via dynamical relaying," Phys. Rev. Lett. 97, 123902.

Flunkert, V., O. D’Huys, J. Danckaert, I. Fischer, and E. Schöll, 2009, "Bubbling in delay-coupled lasers," Phys. Rev. E 79, 065201.

Flunkert, V., and E. Schöll, 2007, "Suppressing noise-induced intensity pulsations in semiconductor lasers by means of timedelayed feedback," Phys. Rev. E 76, 066202.

Flunkert, V., and E. Schöll, 2012, "Chaos synchronization in networks of delay-coupled lasers: role of the coupling phases," New J. Phys. 14, 033039.

Flunkert, V., S. Yanchuk, T. Dahms, and E. Schöll, 2010, "Synchronizing distant nodes: a universal classification of networks," Phys. Rev. Lett. 105, 254101.

Fujino, H., and J. Ohtsubo, 2001, "Synchronization of chaotic oscillations in mutually coupled semiconductor lasers," Opt. Rev. 8, 351.

Fujiwara, Natsuki, Yoshiro Takiguchi, and Junji Ohtsubo, 2003, "Observation of the synchronization of chaos in mutually injected vertical-cavity surface-emitting semiconductor lasers.," Opt. Lett. 28, 1677.

Gabriel, C., C. Wittmann, D. Sych, R. Dong, W. Mauerer, U. L. Andersen, C. Marquardt, and G. Leuchs, 2010, "A generator for unique quantum random numbers based on vacuum states," Nat. Photonics 4, 711.

Gammaitoni, Luca, Peter Hänggi, Peter Jung, and Fabio Marchesoni, 1998, "Stochastic resonance," Rev. Mod. Phys. 70, 223.

Gang, Hu, T. Ditzinger, C.Z. Ning, and H. Haken, 1993, "Stochastic resonance without external periodic force," Phys. Rev. Lett. 71, 807.

Garcia-Ojalvo, J., J. Casademont, M. C. Torrent, C. R. Mirasso, and J. M. Sancho, 1999, "Coherence and synchronization in diodelaser arrays with delayed global coupling," Int. J. Bifurcation Chaos Appl. Sci. Eng. 09, 2225.

Garcia-Ojalvo, J., and R. Roy, 2001, "Spatiotemporal communication with synchronized optical chaos," Phys. Rev. Lett. 86, 5204.

Gavrielides, Athanasios, David W. Sukow, Guinevere Burner, Taylor McLachlan, John Miller, and Jake Amonette, 2010, 
"Simple and complex square waves in an edge-emitting diode laser with polarization-rotated optical feedback," Phys. Rev. E 81, 056209 .

Giacomelli, G., M. Giudici, S. Balle, and J. R. Tredicce, 2000, "Experimental evidence of coherence resonance in an optical system," Phys. Rev. Lett. 84, 3298.

Giacomelli, G., and A. Politi, 1996, "Relationship between delayed and spatially extended dynamical systems," Phys. Rev. Lett. 76, 2686.

Girvan, M., and M.E. J. Newman, 2002, "Community structure in social and biological networks," Proc. Natl. Acad. Sci. U.S.A. 99, 7821.

Giuliani, G., M. Norgia, S. Donati, and T. Bosch, 2002, "Laser diode self-mixing technique for sensing applications,” J. Opt. A 4, S283.

Goedgebuer, J. P., Laurent Larger, and Henri Porte, 1998, “Optical cryptosystem based on synchronization of hyperchaos generated by a delayed feedback tunable laser diode," Phys. Rev. Lett. 80, 2249.

Goedgebuer, J.-P., L. Larger, H. Porte, and F. Delorme, 1998, "Chaos in wavelength with a feedback tunable laser diode," Phys. Rev. E 57, 2795.

Gollo, Leonardo L., Claudio Mirasso, and Alessandro E. P. Villa, 2010, "Dynamic control for synchronization of separated cortical areas through thalamic relay," NeuroImage 52, 947.

Gollo, Leonardo L., Claudio R. Mirasso, Mercedes Atienza, Maite Crespo-Garcia, and Jose L. Cantero, 2011, "Theta band zero-lag long-range cortical synchronization via hippocampal dynamical relaying," PLoS ONE 6, e17756.

Gonzalez, C. M., M. C. Soriano, M. C. Torrent, J. Garcia-Ojalvo, and I. Fischer, 2011, "Dynamical and Synchronization Properties of Delay-Coupled Lasers," Nonlinear Laser Dynamics: From Quantum Dots to Cryptography (Wiley-VCH Verlag $\mathrm{GmbH} \&$ Co. KGaA, Weinheim), p. 217.

González, C. M., J. M. Buldú, M. C. Torrent, and J. García-Ojalvo, 2007, "Processing distributed inputs in coupled excitable lasers," Phys. Rev. A 76, 053824.

González, C. M., C. Masoller, M. C. Torrent, and J. García-Ojalvo, 2007, "Synchronization via clustering in a small delay-coupled laser network," Europhys. Lett. 79, 64003.

Gonzalez, M.C., M.C. Torrent, and J. García-Ojalvo, 2007, "Controlling the leader-laggard dynamics in delay-synchronized lasers," Chaos 17, 033122.

Gray, George. R., Andrew T. Ryan, Govind P. Agrawal, and Edward C. Gage, 1993, "Control of optical-feedback-induced laser intensity noise in optical data recording," Opt. Eng. 32, 739.

Green, Kirk, and Bernd Krauskopf, 2004, "Bifurcation analysis of a semiconductor laser subject to non-instantaneous phase-conjugate feedback," Opt. Commun. 231, 383.

Guo, Shang Jiang, and Li Hong Huang, 2007, "Pattern Formation and Continuation in a Trineuron Ring with Delays," Acta Math. Sin. 23, 799.

Haegeman, B., K. Engelborghs, D. Roose, D. Pieroux, and T. Erneux, 2002, "Stability and rupture of bifurcation bridges in semiconductor lasers subject to optical feedback," Phys. Rev. E 66, 046216.

Hall, R. N., G.E. Fenner, J.D. Kingsley, T. J. Soltys, and R. O. Carlson, 1962, "Coherent light emission from GaAs junctions," Phys. Rev. Lett. 9, 366.

Hegarty, S. P., D. Goulding, B. Kelleher, G. Huyet, M. T. Todaro, A. Salhi, A. Passaseo, and M. De Vittorio, 2007, "Phase-locked mutually coupled $1.3 \mu \mathrm{m}$ quantum-dot lasers," Opt. Lett. 32, 3245.

Hegarty, S.P., G. Huyet, and J. G. McInerney, 1999, "Pattern formation in the transverse section of a laser with a large Fresnel number," Phys. Rev. Lett. 82, 1434.

Heil, T., I. Fischer, W. Elsässer, J. Mulet, and C. R. Mirasso, 1999, "Statistical properties of low-frequency fluctuations during single-mode operation in distributed-feedback lasers: experiments and modeling," Opt. Lett. 24, 1275.

Heil, T., I. Fischer, and W. Elsäßer, 1999, "Influence of amplitudephase coupling on the dynamics of semiconductor lasers subject to optical feedback," Phys. Rev. A 60, 634.

Heil, T., I. Fischer, and W. Elsäßer, 2000, "Stabilization of feedbackinduced instabilities in semiconductor lasers," J. Opt. B 2, 413.

Heil, T., I. Fischer, W. Elsäßer, and A. Gavrielides, 2001, "Dynamics of Semiconductor Lasers Subject to Delayed Optical Feedback: The Short Cavity Regime,' Phys. Rev. Lett. 87, 243901.

Heil, T., I. Fischer, W. Elsäßer, B. Krauskopf, K. Green, and A. Gavrielides, 2003, "Delay dynamics of semiconductor lasers with short external cavities: Bifurcation scenarios and mechanisms," Phys. Rev. E 67, 066214.

Heil, T., J. Mulet, I. Fischer, C. R. Mirasso, M. Peil, P. Colet, and W. Elsässer, 2002, "ON/OFF phase shift keying for chaos-encrypted communication using external-cavity semiconductor lasers," IEEE J. Quantum Electron. 38, 1162.

Heil, T., A. Uchida, P. Davis, and T. Aida, 2003, "TE-TM dynamics in a semiconductor laser subject to polarization-rotated optical feedback," Phys. Rev. A 68, 033811.

Heil, Tilmann, Ingo Fischer, Wolfgang Elsäßer, Josep Mulet, and Claudio R. Mirasso, 2001, "Chaos synchronization and spontaneous symmetry-breaking in symmetrically delay-coupled semiconductor lasers," Phys. Rev. Lett. 86, 795.

Heiligenthal, Sven, Thomas Dahms, Serhiy Yanchuk, Thomas Jüngling, Valentin Flunkert, Ido Kanter, Eckehard Schöll, and Wolfgang Kinzel, 2011, "Strong and Weak Chaos in Nonlinear Networks with Time-Delayed Couplings," Phys. Rev. Lett. 107, 234102.

Henry, C., and R. Kazarinov, 1986, "Instability of semiconductor lasers due to optical feedback from distant reflectors," IEEE J. Quantum Electron. 22, 294.

Henry, C.H., 1982, "Theory of the linewidth of semiconductor lasers,” IEEE J. Quantum Electron. 18, 259.

Hess, O., S. W. Koch, and J. V. Moloney, 1995, "Filamentation and beam propagation in broad-area semiconductor lasers," IEEE J. Quantum Electron. 31, 35.

Hess, O., and T. Kuhn, 1996, "Maxwell-Bloch equations for spatially inhomogeneous semiconductor lasers. i: Theoretical description," Phys. Rev. A 54, 3347.

Hess, O., and E. Schöll, 1994, "Spatio-temporal dynamics in twinstripe semiconductor lasers," Physica (Amsterdam) 70D, 165.

Hicke, K., O. D’Huys, V. Flunkert, E. Schöll, J. Danckaert, and I. Fischer, 2011, "Mismatch and synchronization: Influence of asymmetries in systems of two delay-coupled lasers," Phys. Rev. E 83, 056211.

Hirano, K., T. Yamazaki, S. Morikatsu, H. Okumura, H. Aida, A. Uchida, S. Yoshimori, K. Yoshimura, T. Harayama, and P. Davis, 2010, "Fast random bit generation with bandwidth-enhanced chaos in semiconductor lasers," Opt. Express 18, 5512.

Hohl, A., T. Gavrielides, T. Erneux, and V. Kovanis, 1997, "Localized synchronization in two coupled nonidentical semiconductor lasers," Phys. Rev. Lett. 78, 4745.

Hohl, A., H. J. van der Linden, and R. Roy, 1995, "Determinism and stochasticity of power-dropout events in semiconductor lasers with optical feedback, “ Opt. Lett. 20, 2396.

Hohl, Angela, and Athanasios Gavrielides, 1999, "Bifurcation Cascade in a Semiconductor Laser Subject to Optical Feedback," Phys. Rev. Lett. 82, 1148.

Holman, W. T., J. A. Connelly, and A. B. Dowlatabadi, 1997, "An integrated analog/digital random noise source," IEEE Trans. Circuits Syst. I Fundam. Theory Appl. 44, 521. 
Hong, Y., M. W. Lee, J. Paul, P. S. Spencer, and K. A. Shore, 2009, " $\mathrm{GHz}$ bandwidth message transmission using chaotic verticalcavity surface-emitting lasers," J. Lightwave Technol. 27, 5099.

Huang, D., et al., 1991, "Optical coherence tomography," Science 254, 1178.

Huyet, G., 1998, "Low frequency fluctuations and multimode operation of a semiconductor laser with optical feedback," Opt. Commun. 149, 341.

Huyet, G., J. K. White, A. J. Kent, S. P. Hegarty, J. V. Moloney, and J. G. Mcinerney, 1999, "Dynamics of a semiconductor laser with optical feedback,” Phys. Rev. A 60, 1534.

Ikeda, K., and K. Matsumoto, 1987, "High-dimensional chaotic behavior in systems with time-delayed feedback," Physica (Amsterdam) 29D, 223.

Ikeda, K., K. Otsuka, and K. Matsumoto, 1989, "Maxwell-bloch turbulence," Prog. Theor. Phys. Suppl. 99, 295.

Ikeda, Kensuke, 1979, "Multiple-Valued Stationary State and its Instability of the Transmitted Light by a Ring Cavity System," Opt. Commun. 30, 257.

In, Visarath, Patrick Longhini, and Antonio Palacios, 2009, Eds., Applications of Nonlinear Dynamics: Model and Design of Complex Systems (Springer-Verlag, Berlin).

Jaeger, H., and H. Haas, 2004, "Harnessing nonlinearity: Predicting chaotic systems and saving energy in wireless communication," Science 304, 78.

Javaloyes, J., P. Mandel, and D. Pieroux, 2003, "Dynamical properties of lasers coupled face to face," Phys. Rev. E 67, 036201.

Jiang, N., W. Pan, L. Yan, B. Luo, W. Zhang, S. Xiang, L. Yang, and D. Zheng, 2010, "Chaos synchronization and communication in mutually coupled semiconductor lasers driven by a third laser," J. Lightwave Technol. 28, 1978.

Jiang, Shijun, Mario Dagenais, and R. A. Morgan, 1995, "Spectral characteristics of vertical-cavity surface-emitting lasers with strong external optical feedback," IEEE Photonics Technol. Lett. 7, 739.

Just, Wolfram, Axel Pelster, Michael Schanz, and Eckehard Schöll, 2010, “Theme issue 'delayed complex systems',' Phil. Trans. R. Soc. A 368, 303.

Kanter, I., M. Butkovski, Y. Peleg, M. Zigzag, Y. Aviad, I. Reidler, M. Rosenbluh, and W. Kinzel, 2010, "Synchronization of random bit generators based on coupled chaotic lasers and application to cryptography," Opt. Express 18, 18292.

Kanter, I., E. Kopelowitz, R. Vardi, M. Zigzag, W. Kinzel, M. Abeles, and D. Cohen, 2011, "Nonlocal mechanism for cluster synchronization in neural circuits," Europhys. Lett. 93, 66001.

Kanter, I., M. Zigzag, A. Englert, F. Geissler, and W. Kinzel, 2011, "Synchronization of unidirectional time delay chaotic networks and the greatest common divisor," Europhys. Lett. 93, 60003.

Kanter, Ido, Yaara Aviad, Igor Reidler, Elad Cohen, and Michael Rosenbluh, 2010, "An optical ultrafast random bit generator," Nat. Photonics 4, 58.

Kanter, Ido, Evi Kopelowitz, and Wolfgang Kinzel, 2008, "Public channel cryptography: Chaos synchronization and Hilbert's tenth problem," Phys. Rev. Lett. 101, 084102.

Kestler, Johannes, Wolfgang Kinzel, and Ido Kanter, 2007, "Sublattice synchronization of chaotic networks with delayed couplings,” Phys. Rev. E 76, 035202.

Kestler, Johannes, Evi Kopelowitz, Ido Kanter, and Wolfgang Kinzel, 2008, "Patterns of chaos synchronization," Phys. Rev. E 77, 046209.

Khaykovich, Lev, Tal Galfsky, Zav Shotan, and Noam Gross, 2009, "TE-TM coupled mode dynamics in a semiconductor laser subject to feedback with variably rotated polarization," Opt. Commun. 282, 2059.
Kim, M.-Y., R. Roy, J. L. Aron, and I. B. Carr, T. W. Schwartz, 2005, "Scaling behavior of laser population dynamics with time-delayed coupling: Theory and experiment," Phys. Rev. Lett. 94, 088101.

Kinzel, W., A. Englert, G. Reents, M. Zigzag, and I. Kanter, 2009, "Synchronization of networks of chaotic units with time-delayed couplings," Phys. Rev. E 79, 056207.

Kinzel, W., and I. Kanter, 2008, "Secure Communication with Chaos Synchronization," Handbook of Chaos Control (WileyVCH Verlag GmbH \& Co. KGaA, Weinheim), 2nd ed., p. 303.

Klein, Einat, Noam Gross, Evi Kopelowitz, Michael Rosenbluh, Lev Khaykovich, Wolfgang Kinzel, and Ido Kanter, 2006, "Publicchannel cryptography based on mutual chaos pass filters," Phys. Rev. E 74, 046201.

Klein, Einat, Noam Gross, Michael Rosenbluh, Wolfgang Kinzel, Lev Khaykovich, and Ido Kanter, 2006, "Stable isochronal synchronization of mutually coupled chaotic lasers," Phys. Rev. E 73, 066214.

Kolachevsky, N., J. Alnis, C. G. Parthey, A. Matveev, R. Landig, and T. W. Hänsch, 2011, "Low phase noise diode laser oscillator for 1S-2S spectroscopy in atomic hydrogen," Opt. Lett. 36, 4299.

Kosonocky, W., and R. Cornely, 1968, "GaAs laser amplifiers," IEEE J. Quantum Electron. 4, 125.

Kowarschik, R., T. Tschudi, and L. Wang, 2001, Coherent Optical Metrology (Wiley-VCH, Weinham).

Kozyreff, G., A. Vladimirov, and P. Mandel, 2000, "Global coupling with time delay in an array of semiconductor lasers," Phys. Rev. Lett. 85, 3809.

Kozyreff, G., A. Vladimirov, and Paul Mandel, 2001, "Dynamics of a semiconductor laser array with delayed global coupling," Phys. Rev. E 64, 016613.

Krauskopf, Bernd, George R Gray, and Daan Lenstra, 1998, "Semiconductor laser with phase-conjugate feedback: Dynamics and bifurcations," Phys. Rev. E 58, 7190.

Krauskopf, Bernd, and Daan Lenstra, 2000, Eds., Fundamental Issues of Nonlinear Laser Dynamics, AIP Conf. Proc. No. 548 (Melville, New York).

Kroemer, Herbert, 2001, "Nobel Lecture: Quasielectric fields and band offsets: teaching electrons new tricks," Rev. Mod. Phys. 73, 783.

Kürz, Peter, Ron Nagar, and Takaaki Mukai, 1996, "Highly efficient phase conjugation using spatially nondegenerate four-wave mixing in a broad-area laser diode," Appl. Phys. Lett. 68, 1180.

Kusumoto, K., and J. Ohtsubo, 2002, "1.5 GHz message transmission based on synchronization of chaos in semiconductor lasers," Opt. Lett. 27, 989.

Lakshmanan, M., and D.V. Senthilkumar, 2010, Dynamics of Nonlinear Time-Delay Systems, Springer Series in Synergetics (Springer, New York).

Landsman, A.S., and I. B. Schwartz, 2007, "Complete chaotic synchronization in mutually coupled time-delay systems," Phys. Rev. E 75, 026201.

Lang, R., and K. Kobayashi, 1980, "External optical feedback effects on semiconductor injection laser properties," IEEE J. Quantum Electron. 16, 347.

Larger, L., M. C. Soriano, D. Brunner, L. Appeltant, J. M. Gutierrez, L. Pesquera, C. R. Mirasso, and I. Fischer, 2012, "Photonic information processing beyond Turing: an optoelectronic implementation of reservoir computing," Opt. Express 20, 3241.

Larger, Laurent, and John M. Dudley, 2010, "Nonlinear dynamics: Optoelectronic chaos," Nature (London) 465, 41.

Lasher, G. J., 1964, "Analysis of a proposed bistable injection laser," Solid State Electron. 7, 707.

Lavrov, R., M. Jacquot, and L. Larger, 2010, "Nonlocal nonlinear electro-optic phase dynamics demonstrating $10 \mathrm{~Gb} / \mathrm{s}$ chaos communications,” IEEE J. Quantum Electron. 46, 1430. 
Lawrence, J., and D. Kane, 2001, "Contrasting conventional optical and phase-conjugate feedback in laser diodes," Phys. Rev. A 63, 033805.

Le Berre, M., E. Ressayre, A. Tallet, and H.M. Gibbs, 1987, "Conjecture on the dimensions of chaotic attractors of delayedfeedback dynamical systems," Phys. Rev. A 35, 4020.

Lee, M. W., Y. Hong, and K. A. Shore, 2004, "Experimental demonstration of VCSEL-based chaotic optical communications," IEEE Photonics Technol. Lett. 16, 2392.

Lenstra, D., B. H. Verbeek, and A. J. den Boef, 1985, "Coherence collapse in single-mode semiconductor lasers due to optical feedback," IEEE J. Quantum Electron. 21, 674.

Levine, A.M., G.H.M Van Tartwijk, D. Lenstra, and T. Erneux, 1995, "Diode lasers with optical feedback: Stability of the maximum gain mode," Phys. Rev. A 52, R3436.

Li, C., X. Liao, and K. Wong, 2004, "Chaotic lag synchronization of coupled time-delayed systems and its applications in secure communication," Physica (Amsterdam) 194D, 187.

Li, Hua, Angela Hohl, and Athanasios Gavrielides, 1998, "Stable polarization self-modulation in vertical-cavity surface-emitting lasers," Appl. Phys. Lett. 72, 2355.

Li, X.F., W. Pan, B. Luo, D. Ma, and W.L. Zhang, 2007, "Nonlinear dynamics and localized synchronization in mutually coupled VCSELs," Opt. Laser Technol. 39, 875.

Lin, F. Y., and J. M. Liu, 2004, "Chaotic lidar," IEEE J. Sel. Top. Quantum Electron. 10, 991.

Lin, Fan-Yi, and J.M. Liu, 2003, "Nonlinear dynamics of a semiconductor laser with delayed negative optoelectronic feedback," IEEE J. Quantum Electron. 39, 562.

Lindley, Brandon. S., and Ira B Schwartz, 2011, "Modeling synchronization in networks of delay-coupled fiber ring lasers.," Opt. Express 19, 24460.

Liu, J., H. Chen, and S. Tang, 2002, "Synchronized chaotic optical communications at high bit rates," IEEE J. Quantum Electron. 38, 1184.

Liu, Y., H.F. Chen, J.M. Liu, P. Davis, and T. Aida, 2001, "Communication using synchronization of optical-feedbackinduced chaos in semiconductor lasers," IEEE Trans. Circuits Syst. I, Fundam. Theory Appl.48, 1484.

Liu, Y., P. Davis, and Y. Takiguchi, 1999, "Recovery process of low-frequency fluctuations in laser diodes with external optical feedback, “ Phys. Rev. E 60, 6595.

Liu, Y., and J. Ohtsubo, 1991, "Observation of higher harmonic bifurcations in a chaotic system using a laser diode active interferometer," Opt. Commun. 85, 457.

Liu, Y., Y. Takiguchu, P. Davis, T. Aida, and S. Saito, 2002, "Experimental observation of complete chaos synchronization in semiconductor lasers," Appl. Phys. Lett. 80, 4306.

Locquet, A., C. Masoller, and C. R. Mirasso, 2002, "Synchronization regimes of optical-feedback-induced chaos in unidirectionally coupled semiconductor lasers," Phys. Rev. E 65, 056205.

Loh, W.H., Y. Ozeki, and C.L. Tang, 1990, "High-frequency polarization self-modulation and chaotic phenomena in external cavity semiconductor lasers," Appl. Phys. Lett. 56, 2613.

Lüdge, Kathy, 2011, Ed., Nonlinear Laser Dynamics: From Quantum Dots to Cryptography, Reviews of Nonlinear Dynamics and Complexity (Wiley-VCH Verlag $\mathrm{GmbH} \& \mathrm{Co}$. KGaA, Weinheim, Germany), Vol. 5.

Mackey, Michael. C., and Leon Glass, 1977, "Oscillations and chaos in physiological control systems," Science 197, 287.

Mandre, S., I. Fischer, and W. Elsäßer, 2005, "Spatiotemporal emission dynamics of a broad-area semiconductor laser in an external cavity: stabilization and feedback-induced instabilities," Opt. Commun. 244, 355.
Mandre, Shyam. K., Ingo Fischer, and Wolfgang Elsässer, 2003, "Control of the spatiotemporal emission of a broad-area semiconductor laser by spatially filtered feedback," Opt. Lett. 28, 1135.

Marchenko, Y. I., 1967, "Mutual synchronization of self-oscillating systems with consideration of the delay in interaction forces," Radiophys. Quantum Electron. 10, 861.

Marchenko, Yu I., and V.P. Rubanik, 1965, "Mutual Synchronization of Molecular Oscillators," Sov. Radiophys. 8, 481.

Marino, Francesco, Massimo Giudici, Stéphane Barland, and Salvador Balle, 2002, "Experimental Evidence of Stochastic Resonance in an Excitable Optical System," Phys. Rev. Lett. 88, 040601.

Maritan, Amos, and Jayanth R. Banavar, 1994, "Chaos, noise, and synchronization,” Phys. Rev. Lett. 72, 1451.

Marsaglia, G., 1995, "Diehard: A Battery of Tests of Randomness," http://www.stat.fsu.edu/pub/diehard/.

Martín-Regalado, J., G. H. M. van Tartwijk, S. Balle, and M. San Miguel, 1996, "Mode control and pattern stabilization in broadarea lasers by optical feedback," Phys. Rev. A 54, 5386.

Martinez Avila, Jhon F., and J. R. Rios Leite, 2009, "Time delays in the synchronization of chaotic coupled lasers with feedback," Opt. Express 17, 21442.

Martinez Avila, Jhon F., Hugo L. D. de S. Cavalcante, and J. Rios Leite, 2004, "Experimental Deterministic Coherence Resonance," Phys. Rev. Lett. 93, 144101.

Masoller, C., 2001, "Anticipation in the synchronization of chaotic semiconductor lasers with optical feedback," Phys. Rev. Lett. 86, 2782.

Masoller, C., 2002, "Noise-Induced Resonance in Delayed Feedback Systems,” Phys. Rev. Lett. 88, 034102.

Melnik, Sergey, Guillaume Huyet, and Alexander Uskov, 2006, "The linewidth enhancement factor alpha of quantum dot semiconductor lasers," Opt. Express 14, 2950.

Merbach, D., O. Hess, H. Herzel, and E. Schöll, 1995, "Injectioninduced bifurcations of transverse spatiotemporal patterns in semiconductor laser arrays," Phys. Rev. E 52, 1571.

Meucci, R., A. Di Garbo, E. Allaria, and F. T. Arecchi, 2002, "Autonomous bursting in a homoclinic system," Phys. Rev. Lett. 88, 144101.

Mikami, T., K. Kanno, K. Aoyama, A. Uchida, T. Ikeguchi, T. Harayama, S. Sunada, K. Arai, K. Yoshimura, and P. Davis, 2012, "Estimation of entropy rate in a fast physical random-bit generator using a chaotic semiconductor laser with intrinsic noise," Phys. Rev. E 85, 016211.

Miles, R. O., A. Dandridge, A.B. Tveten, T.G. Giallorenzi, and H.F. Taylor, 1981, "Low-frequency noise characteristics of channel substrate planar GaAlAs laser diodes," Appl. Phys. Lett. 38, 848.

Milo, R., S. Shen-Orr, S. Itzkovitz, N. Kashtan, D. Chklovskii, and U. Alon, 2002, "Network motifs: Simple building blocks of complex networks," Science 298, 824.

Mirasso, C. R., P. Colet, and P. Garcia-Fernandez, 1996, "Synchronization of chaotic semiconductor lasers: application to encoded communications," IEEE Photonics Technol. Lett. 8, 299.

Moloney, J. V., R. A. Indik, J. Hader, and S. W. Koch, 1999, "Modeling semiconductor amplifiers and lasers: from microscopic physics to device simulation,” J. Opt. Soc. Am. B 16, 2023.

Mørk, J., J. Mark, and B. Tromborg, 1990, "Route to chaos and competition between relaxation oscillations for a semiconductor laser with optical feedback," Phys. Rev. Lett. 65, 1999.

Mørk, J., B. Tromborg, and P.L. Christiansen, 1988, "Bistability and low-frequency fluctuations in semiconductor lasers with 
optical feedback: a theoretical analysis," IEEE J. Quantum Electron. 24, 123.

Mørk, J., B. Tromborg, and J. Mark, 1992, "Chaos in semiconductor lasers with optical feedback: theory and experiment," IEEE J. Quantum Electron. 28, 93.

Mulet, J., C. Massoller, and C.R. Mirasso, 2002, "Modeling bidirectionally coupled single-mode semiconductor lasers," Phys. Rev. A 65, 06385.

Mulet, J., C.R. Mirasso, T. Heil, and I. Fischer, 2004, "Synchronization scenario of two distant mutually coupled semiconductor lasers," J. Opt. B 6, 97.

Murakami, A., and J. Ohtsubo, 2002, "Synchronization of feedback-induced chaos in semiconductor lasers by optical injection," Phys. Rev. A 65, 033826.

Murakami, Atsushi, Junji Ohtsubo, and Yun Liu, 1997, "Stability analysis of semiconductor laser with phase-conjugate feedback," IEEE J. Quantum Electron. 33, 1825.

Murphy, T.E., and R. Roy, 2008, "Chaotic lasers: The world's fastest dice," Nat. Photonics 2, 714.

Nathan, M. I., W.P. Dumke, G. Burns, F. H. Dill, and G. Lasher, 1962, "Stimulated emission of radiation from GaAs p-n junctions," Appl. Phys. Lett. 1, 62.

Naumenko, Alexander, Pascal Besnard, and Natalia Loiko, 2003, "Characteristics of a semiconductor laser coupled with a fiber Bragg grating with arbitrary amount of feedback," IEEE J. Quantum Electron. 39, 1216.

Nishikawa, T., and A.E. Motter, 2010, "Network synchronization landscape reveals compensatory structures, quantization, and the positive effect of negative interactions," Proc. Natl. Acad. Sci. U.S.A. 107, 10342.

Nixon, Micha, Moti Fridman, Eitan Ronen, Asher A. Friesem, Nir Davidson, and Ido Kanter, 2012, "Controlling synchronization in large laser networks," Phys. Rev. Lett. 108, 214101.

Nixon, Micha, Moti Friedman, Eitan Ronen, Asher Friesem, Nir Davidson, and Ido Kanter, 2011, "Synchronized Cluster Formation in Coupled Laser Networks," Phys. Rev. Lett. 106, 223901.

Ohtsubo, Junji, 2008, Semiconductor Lasers: Stability, Instability and Chaos, edited by W.T. Rhodes, Springer Series in Optical Sciences (Springer-Verlag, Berlin/Heidelberg), 2nd ed.

Oliver, Neus, Miguel C. Soriano, David W. Sukow, and Ingo Fischer, 2011, "Dynamics of a semiconductor laser with polarization-rotated feedback and its utilization for random bit generation," Opt. Lett. 36, 4632.

Orosz, G., R. E. Wilson, and R. Szalai, 2009, "Exciting traffic jams: Nonlinear phenomena behind traffic jam formation on highways," Phys. Rev. E 80, 046205.

Osborne, S., S. O'Brien, K. Buckley, R. Fehse, A. Amann, J. Patchell, B. Kelly, D. R. Jones, J. O'Gorman, and E. P. O'Reilly, 2007, "Design of single-mode and two-color Fabry-Pérot lasers with patterned refractive index," IEEE J. Sel. Top. Quantum Electron. 13, 1157.

Otsuka, K., and J.L. Chern, 1991, "High-speed picosecond pulse generation in semiconductor lasers with incoherent optical feedback," Opt. Lett. 16, 1759.

Otsuka, Kenju, 2000, Nonlinear Dynamics in Optical Complex Systems, edited by Takeshi Kamiya, Advances in Opto-Electronics (Kluwer Academic Publishers, Dordrecht, The Netherlands).

Ozaki, M., H. Someya, T. Mihara, A. Uchida, S. Yoshimori, K. Panajotov, and M. Sciamanna, 2009, "Leader-laggard relationship of chaos synchronization in mutually coupled vertical-cavity surface-emitting lasers with time delay,” Phys. Rev. E 79, 026210.

Panajotov, K., M. Sciamanna, A. Tabaka, P. Mégret, M. Blondel, G. Giacomelli, F. Marin, H. Thienpont, and I. Veretennicoff, 2004,
"Residence time distribution and coherence resonance of opticalfeedback-induced polarization mode hopping in vertical-cavity surface-emitting lasers,” Phys. Rev. A 69, 011801(R).

Paquot, Y., F. Duport, A. Smerieri, J. Dambre, B. Schrauwen, M. Haelterman, and S. Massar, 2012, "Optoelectronic reservoir computing," Sci. Rep. 2, 287.

Pearson, G. N., P. J. Roberts, J.R. Eacock, and M. Harris, 2002, "Analysis of the performance of a coherent pulsed fiber lidar for aerosol backscatter applications," Appl. Opt. 41, 6442.

Pecora, L. M., and T. L. Carroll, 1990, "Synchronization in chaotic systems,” Phys. Rev. Lett. 64, 821.

Pecora, Louis M., and Thomas L. Carroll, 1991, "Driving Systems with Chaotic Signals," Phys. Rev. A 44, 2374.

Pecora, Louis M., and Thomas L. Carroll, 1998, "Master Stability Functions for Synchronized Coupled Systems," Phys. Rev. Lett. 80, 2109.

Peil, M., I. Fischer, and W. Elsäßer, 2006, "Spectral broadband dynamics of semiconductor lasers with resonant short cavities," Phys. Rev. A 73, 023805.

Peil, M., I. Fischer, W. Elsäßer, S. Bakic, N. Damaschke, C. Tropea, S. Stry, and J. Sacher, 2006, "Rainbow refractometry with a tailored incoherent semiconductor laser source," Appl. Phys. Lett. 89, 091106.

Peil, M., L. Larger, and I. Fischer, 2007, "Versatile and robust chaos synchronization phenomena imposed by delayed shared feedback coupling," Phys. Rev. E 76, 045201(R).

Perlikowski, P., S. Yanchuk, O. Popovych, and P. Tass, 2010, "Periodic patterns in a ring of delay-coupled oscillators," Phys. Rev. E 82, 036208.

Petermann, K., 1995, "External optical feedback phenomena in semiconductor lasers," IEEE J. Sel. Top. Quantum Electron. 1, 480.

Pikovsky, A., M. Rosemblum, and J. Kurths, 2001, Synchronization: A Universal Concept in Nonlinear Sciences, Cambridge Nonlinear Science Series (Cambridge University Press, Cambridge, England).

Pikovsky, Arkady, and Jürgen Kurths, 1997, "Coherence Resonance in a Noise-Driven Excitable System,” Phys. Rev. Lett. 78, 775.

Pisarchik, A. N., and F. R. Ruiz-Oliveras, 2010, "Optical chaotic communication using generalized and complete synchronization," IEEE J. Quantum Electron. 46, 279.

Quist, T. M., R. H. Rediker, R. J. Keyes, W.E. Krag, B. Lax, A. L. McWhorter, and H.J. Zeigler, 1962, "Semiconductor maser of GaAs," Appl. Phys. Lett. 1, 91.

Rabinovich, M., R. Huerta, and G. Laurent, 2008, "Transient dynamics for neural processing," Science 321, 48.

Ravoori, Bhargava, Adam Cohen, Jie Sun, Adilson Motter, Thomas Murphy, and Rajarshi Roy, 2011, "Robustness of Optimal Synchronization in Real Networks," Phys. Rev. Lett. 107, 034102.

Ray, Will, Wing-Shun Lam, Parvez Guzdar, and Rajarshi Roy, 2006, "Observation of chaotic itinerancy in the light and carrier dynamics of a semiconductor laser with optical feedback," Phys. Rev. E 73, 026219.

Reidler, I., Y. Aviad, M. Rosenbluh, and I. Kanter, 2009, "Ultrahigh-speed random number generation based on a chaotic semiconductor laser," Phys. Rev. Lett. 103, 024102.

Revuelta, J., C. R. Mirasso, P. Colet, and L. Pesquera, 2002, “Criteria for chaos synchronization of coupled chaotic external-cavity semiconductor lasers," IEEE Photonics Technol. Lett. 14, 140.

Ries, R., and F. Sporleder, 1982, in Proceedings of the 8th European Conference on Optical Communication, Cannes, France, p. 185.

Risch, C., C. Voumard, F. Reinhart, and R. Salathe, 1977, "External-cavity-induced nonlinearities in the light versus current 
characteristic of ( $\mathrm{Ga}, \mathrm{Al})$ As continuous-wave diode lasers," IEEE J. Quantum Electron. 13, 692.

Risch, C. h., and C. Voumard, 1977, "Self-pulsation in the output intensity and spectrum of GaAs-AlGaAs cw diode lasers coupled to a frequency-selective external optical cavity," J. Appl. Phys. 48, 2083.

Roelfsema, Pieter R., Andreas K. Engel, Peter Konig, and Wolf Singer, 1997, "Visuomotor integration is associated with zero time-lag synchronization among cortical areas," Nature (London) 385, 157.

Rogers-Dakin, E. A., J. García-Ojalvo, D. J. DeShazer, and R. Roy, 2006, "Synchronization and symmetry breaking in mutually coupled fiber lasers," Phys. Rev. E 73, 045201(R).

Rogister, F., and M. Blondel, 2004, "Dynamics of two mutually delay-coupled semiconductor lasers," Opt. Commun. 239, 173.

Rogister, F., A. Locquet, D. Pieroux, M. Sciamanna, O. Deparis, P. Megret, and M. Blondel, 2001, "Secure communication scheme using chaotic laser diodes subject to incoherent optical feedback and incoherent optical injection," Opt. Lett. 26, 1486.

Ronen, E., M. Fridman, M. Nixon, A. A. Friesem, and N. Davidson, 2008, "Phase locking of lasers with intracavity polarization elements," Opt. Lett. 33, 2305.

Roy, R., and K. S. Thornburg, Jr., 1994, "Experimental synchronization of chaotic lasers," Phys. Rev. Lett. 72, 2009.

Ruiz-Oliveras, F., A. N. Pisarchik, C. R. Mirasso, and M. C. Soriano, 2011, "Phase-dependent synchronization of coupled semiconductor lasers," Nonlinear Sci. Lett. B 1, 69 [http://www.nonlinearscience .com/paper.php?pid=0000000081].

Ruiz-Oliveras, F. R., and A. N. Pisarchik, 2009, "Synchronization of semiconductor lasers with coexisting attractors," Phys. Rev. E 79, 016202.

Rukhin, Andrew, et al., 2010, "A statistical test suite for random and pseudorandom number generators for cryptographic applications," NIST Special Publication 800-22, Revision 1a.

Sánchez, E., M. A. Matías, and V. Pérez-Muñuzuri, 1997, “Analysis of synchronization of chaotic systems by noise: An experimental study," Phys. Rev. E 56, 4068.

Sanchez-Diaz, A., C. R. Mirasso, P. Colet, and P. Garcia-Fernandez, 1999, "Encoded Gbit/s digital communications with synchronized chaotic semiconductor lasers," IEEE J. Quantum Electron. 35, 292.

Sano, T., 1994, "Antimode dynamics and chaotic itinerancy in the coherence collapse of semiconductor lasers with optical feedback," Phys. Rev. A 50, 2719.

Scalise, L., Y. Yu, G. Giuliani, G. Plantier, and T. Bosch, 2004, "Self-mixing laser diode velocimetry: application to vibration and velocity measurement," IEEE Trans. Instrum. Meas. 53, 223.

Schikora, S., P. Hövel, H. J. Wünsche, E. Schöll, and F. Henneberger, 2006, "All-optical noninvasive control of unstable steady states in a semiconductor laser,” Phys. Rev. Lett. 97, 213902.

Schuster, H. G., and P. Wagner, 1989, "Mutual entrainment of two limit cycle oscillators with time delayed coupling," Prog. Theor. Phys. 81, 939.

Schwartz, IB., and L. B. Shaw, 2007, "Isochronal synchronization of delay-coupled systems," Phys. Rev. E 75, 046207.

Scire, A., J. Mulet, C. R. Mirasso, J. Danckaert, and M. San Miguel, 2003, "Polarization message encoding through vectorial chaos synchronization in vertical-cavity surface-emitting lasers," Phys. Rev. Lett. 90, 113901.

Sherman, S. M., 2005, "Thalamic relays and cortical functioning," Prog. Brain Res. 149, 107.

Shinohara, S., A. Mochizuki, H. Yoshida, and M. Sumi, 1986, "Laser Doppler velocimeter using the self-mixing effect of a semiconductor laser diode," Appl. Opt. 25, 1417.
Singer, W., 1999, "Neuronal synchrony: a versatile code for the definition of relations?," Neuron 24, 49.

Sivaprakasam, S., 2000, "Message encoding and decoding using chaotic external-cavity diode lasers,' IEEE J. Quantum Electron. 36, 35.

Sivaprakasam, S., J. Paul, P. S. Spencer, P. Rees, and K. A. Shore, 2003, "Nullified time-of-flight lead-lag in synchronization of chaotic external-cavity laser diodes," Opt. Lett. 28, 1397.

Sivaprakasam, S., and K. A. Shore, 1999, "Signal masking for chaotic optical communication using external-cavity diode lasers," Opt. Lett. 24, 1200.

Sondermann, Markus, and Thorsten Ackemann, 2005, "Correlation properties and drift phenomena in the dynamics of vertical-cavity surface-emitting lasers with optical feedback," Opt. Express 13, 2707.

Soriano, M.C., Thomas Berkvens, G. Van der Sande, Guy Verschaffelt, Jan Danckaert, and Ingo Fischer, 2011, "Interplay of Current Noise and Delayed Optical Feedback on the Dynamics of Semiconductor Lasers," IEEE J. Quantum Electron. 47, 368.

Soriano, M.C., P. Colet, and C.R. Mirasso, 2009, "Security implications of open-and closed-loop receivers in all-optical chaosbased communications," IEEE Photonics Technol. Lett. 21, 426.

Soriano, M.C., L. Zunino, O. A. Rosso, I. Fischer, and C.R. Mirasso, 2011, "Time scales of a chaotic semiconductor laser with optical feedback under the lens of a permutation information analysis," IEEE J. Quantum Electron. 47, 252.

Soriano, Miguel. C., Guy Van der Sande, Ingo Fischer, and Claudio R. Mirasso, 2012, "Synchronization in simple network motifs with negligible correlation and mutual information measures," Phys. Rev. Lett. 108, 134101.

Sorrentino, Francesco, and Edward Ott, 2007, "Network synchronization of groups," Phys. Rev. E 76, 056114.

Stepan, Gabor, 2009, “Theme issue 'delay effects in brain dynamics'," Phil. Trans. R. Soc. A 367, 1059.

Strogatz, S.H., 2001, "Exploring complex networks," Nature (London) 410, 268.

Strogatz, Steven. H., Daniel M Abrams, Allan McRobie, Bruno Eckhardt, and Edward Ott, 2005, "Crowd synchrony on the millennium bridge," Nature (London) 438, 43.

Stubkjaer, K., and M. B. Small, 1983, "Feedback-induced noise in index-guided semiconductor lasers and its reduction by modulation," Electron. Lett. 19, 388.

Sugawara, T., M. Tachikawa, and T. Tsukamoto, 1994, "Observation of synchronization in laser chaos," Phys. Rev. Lett. 72, 3502.

Sukow, D., T. Heil, I. Fischer, A. Gavrielides, A. Hohl-AbiChedid, and W. Elsäßer, 1999, "Picosecond intensity statistics of semiconductor lasers operating in the low-frequency fluctuation regime," Phys. Rev. A 60, 667.

Sukow, D. W., and D. J. Gauthier, 2000, "Entraining power-dropout events in an external-cavity semiconductor laser using weak modulation of the injection current," IEEE J. Quantum Electron. 36, 175.

Sunada, Satoshi, Takahisa Harayama, Kenichi Arai, Kazuyuki Yoshimura, Peter Davis, Ken Tsuzuki, and Atsushi Uchida, 2011, "Chaos laser chips with delayed optical feedback using a passive ring waveguide.," Opt. Express 19, 5713.

Tabaka, Andrzej, Michael Peil, Marc Sciamanna, Ingo Fischer, Wolfgang Elsäßer, Hugo Thienpont, Irina Veretennicoff, and Krassimir Panajotov, 2006, "Dynamics of vertical-cavity surfaceemitting lasers in the short external cavity regime: Pulse packages and polarization mode competition," Phys. Rev. A 73, 013810.

Tachikawa, Tomokazu, Satoshi Takimoto, Rui Shogenji, and Junji Ohtsubo, 2010, "Dynamics of Broad-Area Semiconductor Lasers 
With Short Optical Feedback," IEEE J. Quantum Electron. 46, 140 .

Tager, A. A., and K. Petermann, 1994, "High-frequency oscillations and self-mode locking in short external-cavity laser diodes," IEEE J. Quantum Electron. 30, 1553.

Takamatsu, Atsuko, Reiko Tanaka, Hiroyasu Yamada, Toshiyuki Nakagaki, Teruo Fujii, and Isao Endo, 2001, "Spatiotemporal Symmetry in Rings of Coupled Biological Oscillators of Physarum Plasmodial Slime Mold," Phys. Rev. Lett. 87, 078102.

Takeuchi, N., N. Sugimoto, H. Baba, and K. Sakurai, 1983, "Random modulation CW lidar," Appl. Opt. 22, 1382.

Takizawa, T., U. Liu, and J. Ohtsubo, 1994, "Chaos in a feedback Fabry-Perot interferometer," IEEE J. Quantum Electron. 30, 334.

Tang, S., and J. M. Liu, 2001a, "Chaotic pulsing and quasi-periodic route to chaos in a semiconductor laser with delayed optoelectronic feedback," IEEE J. Quantum Electron. 37, 329.

Tang, S., and J.M. Liu, 2001b, "Message encoding-decoding at $2.5 \mathrm{Gbits} / \mathrm{s}$ through synchronization of chaotic pulsing semiconductor lasers," Opt. Lett. 26, 1843.

Tang, S., R. Vicente, M. Chiang, C. R. Mirasso, and J. M. Liu, 2004, "Nonlinear dynamics of semiconductor lasers with mutual optoelectronic coupling," IEEE J. Sel. Top. Quantum Electron. 10, 936.

Tanguy, Y., J. Muszalski, J. Houlihan, G. Huyet, E. J. Pearce, P. M. Smowton, and M. Hopkinson, 2004, "Mode formation in broad area quantum dot lasers at $1060 \mathrm{~nm}$," Opt. Commun. 235, 387.

Tanguy, Yann, John Houlihan, Guillaume Huyet, E. A. Viktorov, and Paul Mandel, 2006, "Synchronization and clustering in a multimode quantum dot laser," Phys. Rev. Lett. 96, 053902.

Tauke-Pedretti, Anna, G. Allen Vawter, Erik J. Skogen, Greg Peake, Mark Overberg, Charles Alford, Weng W. Chow, Zhenshan S. Yang, David Torres, and Florante Cajas, 2011, "Mutual Injection Locking of Monolithically Integrated Coupled-Cavity DBR Lasers," IEEE Photonics Technol. Lett. 23, 908.

Taylor, Annette F., Mark R. Tinsley, Fang Wang, Zhaoyang Huang, and Kenneth Showalter, 2009, "Dynamical quorum sensing and synchronization in large populations of chemical oscillators," Science 323, 614.

Terry, J. R., K. S. Thornbug, D. J. DeShazer, G. D. VanWiggeren, S. Q. Zhu, P. Ashwin, and R. Roy, 1999, "Synchronization of chaos in an array of three lasers," Phys. Rev. E 59, 4036.

Tiana-Alsina, J., K. Hicke, X. Porte, M. C. Soriano, M. Torrent, J. Garcia-Ojalvo, and I. Fischer, 2012, "Zero-lag synchronization and bubbling in delay-coupled lasers," Phys. Rev. E 85, 026209.

Tkach, R., and A. R. Chraplyvy, 1986, "Regimes of feedback effects in 1.5- $\mu \mathrm{m}$ distributed feedback lasers," J. Lightwave Technol. 4, 1655.

Toral, R., C. R. Mirasso, E. Hernández-García, and O. Piro, 2001, "Analytical and numerical studies of noise-induced synchronization of chaotic systems," Chaos 11, 665.

Torcini, Alessandro, Stephane Barland, Giovanni Giacomelli, and Francesco Marin, 2006, "Low-frequency fluctuations in vertical cavity lasers: Experiments versus Lang-Kobayashi dynamics," Phys. Rev. A 74, 063801.

Tromborg, B., and J. Mørk, 1990, "Stability analysis and the route to chaos for laser diodes with optical feedback," IEEE Photonics Technol. Lett. 2, 549.

Uchida, A., Y. Liu, I. Fischer, P. Davis, and T. Aida, 2001, "Chaotic antiphase dynamics and synchronization in multimode semiconductor lasers," Phys. Rev. A 64, 023801.

Uchida, Atsushi, 2012, Optical Communication with Chaotic Lasers: Applications of Nonlinear Dynamics and Synchronization (WileyVCH Verlag GmbH \& Co. KGaA, Weinheim, Germany).
Uchida, Atsushi, Ryan McAllister, and Rajarshi Roy, 2004, "Consistency of nonlinear system response to complex drive signals," Phys. Rev. Lett. 93, 244102.

Uchida, Atsushi, Fabien Rogister, Jordi Garcìa-Ojalvo, and Rajarshi Roy, 2005, in Progress in Optics, edited by E. Wolf (Elsevier, New York), Vol. 48, p. 203.

Uchida, Atsushi, et al., 2008, "Fast physical random bit generation with chaotic semiconductor lasers," Nat. Photonics 2, 728.

Ushakov, O., S. Bauer, O. Brox, H.-J. Wünsche, and F. Henneberger, 2004, "Self-Organization in Semiconductor Lasers with Ultrashort Optical Feedback," Phys. Rev. Lett. 92, 043902.

Ushakov, O. V., H.-J. Wünsche, F. Henneberger, I. A. Khovanov, Lutz Schimansky-Geier, and M. A. Zaks, 2005, "Coherence resonance near a hopf bifurcation,” Phys. Rev. Lett. 95, 123903.

Van der Sande, G., M. C. Soriano, I. Fischer, and C. R. Mirasso, 2008, "Dynamics, correlation scaling, and synchronization behavior in rings of delay-coupled oscillators," Phys. Rev. E 77, 055202(R).

Van der Sande, Guy, Miguel C. Soriano, Mirvais Yousefi, Michael Peeters, Jan Danckaert, Guy Verschaffelt, and Daan Lenstra, 2006, "Influence of current noise on the relaxation oscillation dynamics of semiconductor lasers," Appl. Phys. Lett. 88, 071107.

Van der Sande, Guy, Guy Verschaffelt, Jan Danckaert, and Claudio R. Mirasso, 2005, "Ghost stochastic resonance in vertical-cavity surface-emitting lasers: Experiment and theory," Phys. Rev. E 72, 016113

van Tartwijk, G.H.M., and D. Lenstra, 1995, "Semiconductor lasers with optical injection and feedback," Quantum Semiclass. Opt. 7, 87.

van Tartwijk, G.H.M., H.J.C. van der Linden, and D. Lenstra, 1992, "Theory of a diode laser with phase-conjugate feedback," Opt. Lett. 17, 1590.

Van Wiggeren, G.D., and R. Roy, 1998, "Communication with chaotic lasers," Science 279, 1198.

Vardi, R., A. Wallach, E. Kopelowitz, M. Abeles, S. Marom, and I. Kanter, 2012, "Synthetic reverberating activity patterns embedded in networks of cortical neurons," Europhys. Lett. 97, 66002.

Vaschenko, G., M. Giudici, J. Rocca, C. Menoni, J. Tredicce, and S. Balle, 1998, "Temporal Dynamics of Semiconductor Lasers with Optical Feedback,” Phys. Rev. Lett. 81, 5536.

Vaughan, M. P., I. Henning, M. J. Adams, L. J. Rivers, P. Cannard, and I. F. Lealman, 2009, "Mutual optical injection in coupled dbr laser pairs," Opt. Express 17, 2033.

Verstraeten, D., B. Schrauwen, D. Stroobandt, and J. Van Campenhout, 2005, "Isolated word recognition with the liquid state machine: a case study," Inf. Proc. Lett. 95, 521.

Vicente, R., J. Mulet, C. R. Mirasso, and M. Sciamanna, 2006, "Bistable polarization switching in mutually coupled verticalcavity surface-emitting lasers," Opt. Lett. 31, 996.

Vicente, R., T. Perez, and C. R. Mirasso, 2002, "Open-versus closed-loop performance of synchronized chaotic external-cavity semiconductor lasers," IEEE J. Quantum Electron. 38, 1197.

Vicente, R., S. Tang, J. Mulet, C. R. Mirasso, and J. M. Liu, 2004, "Dynamics of semiconductor lasers with bidirectional optoelectronic coupling: Stability, route to chaos and entrainment," Phys. Rev. E 70, 046216.

Vicente, Raul, Leonardo L. Gollo, Claudio R Mirasso, Ingo Fischer, and Gordon Pipa, 2008, "Dynamical relaying can yield zero time lag neuronal synchrony despite long conduction delays.," Proc. Natl. Acad. Sci. U.S.A. 105, 17157.

Vicente, Raúl, Claudio R. Mirasso, and Ingo Fischer, 2007, "Simultaneous bidirectional message transmission in a chaosbased communication scheme.," Opt. Lett. 32, 403. 
Viktorov, E. A., and P. Mandel, 2000, "Low frequency fluctuations in a multimode semiconductor laser with optical feedback.," Phys. Rev. Lett. 85, 3157.

Wang, S. S., and H. G. Winful, 1988, "Dynamics of phase-locked semiconductor-laser arrays," Appl. Phys. Lett. 52, 1774.

Wang, Y., L. Kong, A. Wang, and L. Fan, 2009, "Coherence length tunable semiconductor laser with optical feedback," Appl. Opt. 48, 969.

Wang, Y., B. Wang, and A. Wang, 2008, "Chaotic correlation optical time domain reflectometer utilizing laser diode," IEEE Photonics Technol. Lett. 20, 1636.

Weiss, Carl. O., and Ramon Vilaseca, 1991, Dynamics of Lasers (Wiley-VCH, Weinheim, Germany).

White, J. K., and J. V. Moloney, 1999, "Multichannel communication using an infinite dimensional spatiotemporal chaotic system," Phys. Rev. A 59, 2422.

White, K. J., M. Matus, and J. V. Moloney, 2002, "Achronal generalized synchronization in mutually coupled semiconductor lasers," Phys. Rev. E 65, 036229.

Wieczorek, S., 2009, "Stochastic bifurcation in noise-driven lasers and hopf oscillators," Phys. Rev. E 79, 036209.

Wieczorek, S., B. Krauskopf, and D. Lenstra, 1999, "A unifying view of bifurcations in a semiconductor laser subject to optical injection," Opt. Commun. 172, 279.

Williams, C. R.S., J.C. Salevan, X. Li, R. Roy, and T.E. Murphy, 2010, "Fast physical random number generator using amplified spontaneous emission," Opt. Express 18, 23584.

Winful, H. G., and L. Rahman, 1990, "Synchronized chaos and spatiotemporal chaos in arrays of coupled lasers," Phys. Rev. Lett. 65, 1575 .

Wolff, Sandra, and Andrey Rodionov, 2003, "Fourier-optical transverse mode selection in external-cavity broad-area lasers: experimental and numerical results," IEEE J. Quantum Electron. 39, 448.

Wolfrum, M., 2002, "Instabilities of lasers with moderately delayed optical feedback," Opt. Commun. 212, 127.

Wolfrum, M., and S. Yanchuk, 2006, "Eckhaus instability in systems with large delay," Phys. Rev. Lett. 96, 220201.

Wu, W. T., Y. H. Liao, and F. Y. Lin, 2010, "Noise suppressions in synchronized chaos lidars," Opt. Express 18, 26155.

Wünsche, H.-J., et al., 2005, "Synchronization of delay-coupled oscillators: A study of semiconductor lasers," Phys. Rev. Lett. 94, 163901.

Yamamoto, Y., and T. Kimura, 1981, "Coherent optical fiber transmission systems," IEEE J. Quantum Electron. 17, 919.

Yanchuck, S., K. R. Schneider, and L. Recke, 2004, "Dynamics of two mutually coupled semiconductor lasers: Instantaneous coupling limit," Phys. Rev. E 69, 056221.
Yanchuk, S., and P. Perlikowski, 2009, "Delay and periodicity," Phys. Rev. E 79, 046221.

Yanchuk, S., M. Wolfrum, P. Hövel, and E. Schöll, 2006, “Control of unstable steady states by strongly delayed feedback," Phys. Rev. E 74, 026201.

Yanchuk, Serhiy, Andrzej Stefanski, Tomasz Kapitaniak, and Jerzy Wojewoda, 2006, "Dynamics of an array of mutually coupled semiconductor lasers," Phys. Rev. E 73, 016209.

Ye, Jun, Hua Li, and John G. McInerney, 1993, "Period-doubling route to chaos in a semiconductor laser with weak optical feedback," Phys. Rev. A 47, 2249.

Yeung, M.K.S., and S.H. Strogatz, 1999, "Time delay in the Kuramoto model of coupled oscillators," Phys. Rev. Lett. 82, 648.

Yoshimura, K., J. Muramatsu, P. Davis, T. Harayama, H. Okumura, S. Morikatsu, H. Aida, and A. Uchida, 2012, "Secure key distribution using correlated randomness in lasers driven by common random light," Phys. Rev. Lett. 108, 070602.

Yousefi, M., Y. Barbarin, S. Beri, E. Bente, M. Smit, R. Nötzel, and D. Lenstra, 2007, "New Role for Nonlinear Dynamics and Chaos in Integrated Semiconductor Laser Technology," Phys. Rev. Lett. 98, 044101.

Yousefi, M., D. Lenstra, and G. Vemuri, 2004, "Carrier inversion noise has important influence on the dynamics of a semiconductor laser," IEEE J. Sel. Top. Quantum Electron. 10, 955.

Yousefi, Mirvais, and Daan Lenstra, 1999, "Dynamical behavior of a semiconductor laser with filtered external optical feedback," IEEE J. Quantum Electron. 35, 970.

Yuan, Yuan, and Sue Ann Campbell, 2004, "Stability and synchronization of a ring of identical cells with delayed coupling," J. Dyn. Differ. Equ. 16, 709.

Zamora-Munt, Jordi, C. Masoller, Jordi Garcia-Ojalvo, and Rajarshi Roy, 2010, "Crowd Synchrony and Quorum Sensing in DelayCoupled Lasers,” Phys. Rev. Lett. 105, 264101.

Zhang, W. L., W. Pan, B. Luo, X. H. Zou, and M. Y. Wang, 2008, "One-to-many and many-to-one optical chaos communications using semiconductor lasers," IEEE Photonics Technol. Lett. 20, 712.

Zhou, B. B., and R. Roy, 2007, "Isochronal synchrony and bidirectional communication with delay-coupled nonlinear oscillators," Phys. Rev. E 75, 026205.

Zhou, Changsong, and Jürgen Kurths, 2002, "Noise-induced phase synchronization and synchronization transitions in chaotic oscillators," Phys. Rev. Lett. 88, 230602.

Zigzag, Meital, Maria Butkovski, Anja Englert, Wolfgang Kinzel, and Ido Kanter, 2010, "Zero-lag synchronization and multiple time delays in two coupled chaotic systems," Phys. Rev. E 81, 036215 . 